\title{
SIMULATION IN THE CALL-BY-NEED LAMBDA-CALCULUS WITH LETREC, CASE, CONSTRUCTORS, AND SEQ*
}

\author{
MANFRED SCHMIDT-SCHAUSS ${ }^{a}$, DAVID SABEL $^{b}$, AND ELENA MACHKASOVA $^{c}$ \\ ${ }^{a, b}$ Dept. Informatik und Mathematik, Inst. Informatik, J.W. Goethe-University, PoBox 111932 , \\ D-60054 Frankfurt, Germany \\ e-mail address: \{schauss,sabel\}@ki.informatik.uni-frankfurt.de \\ ${ }^{c}$ Division of Science and Mathematics, University of Minnesota, Morris, MN 56267-2134, U.S.A \\ e-mail address: elenam@morris.umn.edu
}

\begin{abstract}
This paper shows equivalence of several versions of applicative similarity and contextual approximation, and hence also of applicative bisimilarity and contextual equivalence, in LR, the deterministic call-by-need lambda calculus with letrec extended by data constructors, case-expressions and Haskell's seq-operator. LR models an untyped version of the core language of Haskell. The use of bisimilarities simplifies equivalence proofs in calculi and opens a way for more convenient correctness proofs for program transformations.

The proof is by a fully abstract and surjective transfer into a call-by-name calculus, which is an extension of Abramsky's lazy lambda calculus. In the latter calculus equivalence of our similarities and contextual approximation can be shown by Howe's method. Similarity is transferred back to LR on the basis of an inductively defined similarity.

The translation from the call-by-need letrec calculus into the extended call-by-name lambda calculus is the composition of two translations. The first translation replaces the call-by-need strategy by a call-by-name strategy and its correctness is shown by exploiting infinite trees which emerge by unfolding the letrec expressions. The second translation encodes letrec-expressions by using multi-fixpoint combinators and its correctness is shown syntactically by comparing reductions of both calculi.

A further result of this paper is an isomorphism between the mentioned calculi, which is also an identity on letrec-free expressions.
\end{abstract}

2012 ACM CCS: [Theory of computation]: Semantics and reasoning-Program constructs / Program semantics; Logic; [Software and its engineering]: Software notations and tools-Formal language definitions-Semantics.

Key words and phrases: semantics, contextual equivalence, bisimulation, lambda calculus, call-by-need, Haskell.

* This paper is an extended version of [SSSM10] for more expressive calculi, and also of [SS07] w.r.t. infinite trees, with fully worked out proofs.

${ }^{a}$ The first author is supported by the DFG under grant SCHM 986/9-1.

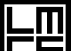

돈 LOGICAL METHODS IN COMPUTER SCIENCE
DOI:10.2168/LMCS-11(1:7)2015 


\section{INTRODUCTION}

Motivation. Non-strict functional programming languages, such as the core-language of Haskell Pey03, can be modeled using extended call-by-need lambda calculi.

The operational semantics of such a programming language defines how programs are evaluated and how the value of a program is obtained. Based on the operational semantics, the notion of contextual equivalence (see e.g. [Mor68, Plo75]) is a natural notion of program equivalence which follows Leibniz's law to identify the indiscernibles, that is two programs are equal iff their observable (termination) behavior is indistinguishable even if the programs are used as a subprogram of any other program (i.e. if the programs are plugged into any arbitrary context). For pure functional programs it suffices to observe whether or not the evaluation of a program terminates with a value (i.e. whether the program converges). Contextual equivalence has several advantages: Any reasonable notion of program equivalence should be a congruence which distinguishes obvious different values, e.g. different constants are distinguished, and functions (abstractions) are distinguished from constants. Contextual equivalence satisfies these requirements and is usually the coarsest of such congruences. Another (general) advantage is that once expressions, contexts, an evaluation, and a set of values are defined in a calculus, its definition of contextual equivalence can be derived, and thus this approach can be used for a broad class of program calculi.

On the other hand, due to the quantification over all program contexts, verifying equivalence of two programs w.r.t. contextual equivalence is often a difficult task. Nevertheless such proofs are required to ensure the correctness of program transformations where the correctness notion means that contextual equivalence is preserved by the transformation. Correctness of program transformations is indispensable for the correctness of compilers, but program transformations also play an important role in several other fields, e.g. in code refactoring to improve the design of programs, or in software verification to simplify expressions and thus to provide proofs or tests.

Bisimulation is another notion of program equivalence which was first invented in the field of process calculi (e.g. [Mil80, Mil99, SW01]), but has also been applied to functional programming and several extended lambda calculi (e.g. [How89, Abr90, How96]). Finding adequate notions of bisimilarity is still an active research topic (see e.g. [KW06, SKS11]). Briefly explained, bisimilarity equates two programs $s_{1}, s_{2}$ if all experiments passed for $s_{1}$ are also passed by $s_{2}$ and vice versa. For applicative similarity (and also bisimilarity) the experiments are evaluation and then recursively testing the obtained values: Abstractions are applied to all possible arguments, data objects are decomposed and the components are tested recursively. Applicative similarity is usually defined co-inductively, i.e. as a greatest fixpoint of an operator. Applicative similarity allows convenient and automatable proofs of correctness of program transformations, e.g. in mechanizing proofs [DBG97].

Abramsky and Ong showed that applicative bisimilarity is the same as contextual equivalence in a specific simple lazy lambda calculus [Abr90, AO93], and Howe [How89, How96] proved that in classes of lambda-calculi applicative bisimulation is the same as contextual equivalence. This leads to the expectation that some form of applicative bisimilarity may be used for calculi with Haskell's cyclic letrec. However, Howe's proof technique appears to be not adaptable to lambda calculi with cyclic let, since there are several deviations from the requirements for the applicability of Howe's framework. (i) Howe's technique is for call-by-name calculi and it is not obvious how to adapt it to call-by-need evaluation. (ii) Howe's technique requires that the values (results of reduction) are recognizable by their 
top operator. This does not apply to calculi with letrec, since letrec-expressions may be values as well as non-values. (iii) Call-by-need calculi with letrec usually require reduction rules to shift and join letrec-bindings. These modifications of the syntactic structure of expressions do not fit well into the proof structure of Howe's method.

Nevertheless, Howe's method is also applicable to calculi with non-recursive let even in the presence of nondeterminism [MSS10], where for the nondeterministic case applicative bisimilarity is only sound (but not complete) w.r.t. contextual equivalence. However, in the case of (cyclic) letrec and nondeterminism applicative bisimilarity is unsound w.r.t. contextual equivalence [SSSM11. This raises a question: which call-by-need calculi with letrec permit applicative bisimilarity as a tool for proving contextual equality?

Our Contribution. In [SSSM10] we have already shown that for the minimal extension of Abramsky's lazy lambda calculus with letrec which implements sharing and explicit recursion, the equivalence of contextual equivalence and applicative bisimilarity indeed holds. However, the full (untyped) core language of Haskell has data constructors, case-expressions and the seq-operator for strict evaluation. Moreover, in SSMS13] it is shown that the extension of Abramsky's lazy lambda calculus with case, constructors, and seq is not conservative, i.e. it does not preserve contextual equivalence of expressions. Thus our results obtained in [SSSM10] for the lazy lambda calculus extended by letrec only are not transferable to the language extended by case, constructors, and seq. For this reason we provide a new proof for the untyped core language of Haskell.

As a model of Haskell's core language we use the call-by-need lambda calculus $L_{L R}$ which was introduced and motivated in SSSS08]. The calculus $L_{L R}$ extends the lazy lambda calculus with letrec-expressions, data constructors, case-expressions for deconstructing the data, and Haskell's seq-operator for strict evaluation.

We define the operational semantics of $L_{L R}$ in terms of a small-step reduction, which we call normal order reduction. As it is usual for lazy functional programming languages, evaluation of $L_{L R}$-expressions successfully halts if a weak head normal form is obtained, i.e. normal order reduction does not reduce inside the body of abstractions nor inside the arguments of constructor applications. The $L_{L R}$ calculus has been studied in detail in SSSS08 and correctness of several important program transformations has been established for it.

Our main result in this paper is that several variants of applicative bisimilarities are sound and complete for contextual equivalence in $L_{L R}$, i.e. coincide with contextual equivalence. Like context lemmas, an applicative bisimilarity can be used as a proof tool for showing contextual equivalence of expressions and for proving correctness of program transformations in the calculus $L_{L R}$. Since we have completeness of our applicative bisimilarities in addition to soundness, our results can also be used to disprove contextual equivalence of expressions in $L_{L R}$. Additionally, our result shows that the untyped applicative bisimilarity is sound for a polymorphic variant of $L_{L R}$, and hence for the typed core language of Haskell.

Having the proof tool of applicative bisimilarity in $L_{L R}$ is also very helpful for more complex calculi if their pure core can be conservatively embedded in the full calculus. An example is our work on Concurrent Haskell [SSS11, SSS12, where our calculus CHF that models Concurrent Haskell has top-level processes with embedded lazy functional evaluation. We have shown in the calculus CHF that Haskell's deterministic core language can be conservatively embedded in the calculus CHF. 


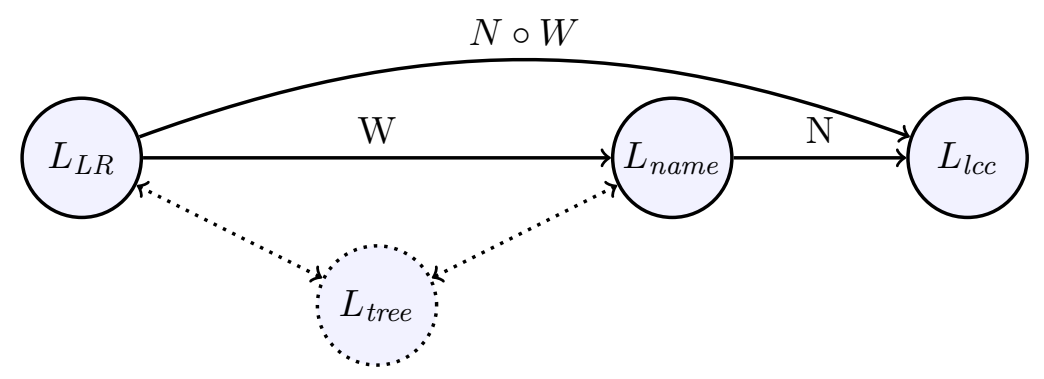

Figure 1: Overall structure. Solid lines are fully-abstract translations, which are also isomorphisms and identities on letrec-free expressions; dotted lines are convergence preservation to/from the system $L_{\text {tree }}$ of infinite trees.

We prove the equivalence between the applicative similarities and contextual equivalence in $L_{L R}$, by lifting the equivalence from a letrec-free call-by-name calculus $L_{l c c}$. The calculus $L_{l c c}$ minimally extends Abramsky's lazy calculus by Haskell's primitives. As shown in [SSMS13], data constructors and seq are explicitly needed in $L_{l c c}$. The structure of the proof, with its intermediate steps, is shown in Figure 1. We prove the equivalence between the applicative similarities and contextual equivalence in $L_{l c c}$, by extending Howe's method. We bridge $L_{L R}$ and $L_{l c c}$ in two steps, using intermediate calculi $L_{\text {name }}$ and $L_{\text {tree }}$. $L_{\text {name }}$ is the call-by-name variant of $L_{L R}$, and $L_{l c c}$ is obtained from $L_{n a m e}$ by encoding letrec using multi-fixpoint combinators. The calculi $L_{L R}$ and $L_{n a m e}$ are related to each other via their infinite unfoldings, thus we introduce a calculus $L_{\text {tree }}$ of infinite trees (similar infinitary rewriting, see [KKSdV97, SS07]). Convergence of expressions in $L_{L R}$ and $L_{n a m e}$ is shown to be equivalent to their translation as an infinite tree in the calculus $L_{\text {tree }}$ (dotted lines in the picture). We establish full abstractness of translation $N$ and $W$ between calculi $L_{L R}, L_{n a m e}$, and $L_{l c c}$ with respect to contextual equivalence. Correctness of similarity is transferred back from $L_{l c c}$ to $L_{L R}$ on the basis of an inductively defined similarity (for more details see Fig. (7.2).

A consequence of our result is that the three calculi $L_{L R}, L_{\text {name }}$, and $L_{l c c}$ are isomorphic, modulo the equivalence (see Corollaries 6.17 and 5.33), and also that the embedding of the calculus $L_{l c c}$ into the call-by-need calculus $L_{L R}$ is an isomorphism of the respective term models.

Related Work. In Gor99 Gordon shows that bisimilarity and contextual equivalence coincide in an extended call-by-name PCF language. Gordon provides a bisimilarity in terms of a labeled transition system. A similar result is obtained in [Pit97] for PCF extended by product types and lazy lists where the proof uses Howe's method ([How89, How96]; see also [MSS10, Pit11]), and where the operational semantics is a big-step one for an extended PCF-language. The observation of convergence in the definition of contextual equivalence is restricted to programs (and contexts) of ground type (i.e. of type integer or Bool). Therefore $\Omega$ and $\lambda x . \Omega$ are equal in the calculi considered by Gordon and Pitts. This does not hold in our setting for two reasons: first, we observe termination for functions and thus the empty context already distinguishes $\Omega$ and $\lambda x . \Omega$, and second, our languages employ Haskell's seq-operator which permits to test convergence of any expression and thus the context seq [.] True distinguishes $\Omega$ and $\lambda x . \Omega$. 
Jef94 presents an investigation into the semantics of a lambda-calculus that permits cyclic graphs, where a fully abstract denotational semantics is described. However, the calculus is different from our calculi in its expressiveness since it permits a parallel convergence test, which is required for the full abstraction property of the denotational model. Expressiveness of programming languages was investigated e.g. in [Fel91] and the usage of syntactic methods was formulated as a research program there, with non-recursive let as the paradigmatic example. Our isomorphism-theorem 7.7 shows that this approach is extensible to a cyclic let.

Related work on calculi with recursive bindings includes the following foundational papers. An early paper that proposes cyclic let-bindings (as graphs) is [AK94, where reduction and confluence properties are discussed. [AFM ${ }^{+}$95, $\mathrm{AF} 97$ study equational theory for call-by-need lambda calculus extended with non-recursive let, which is finer than contextual equivalence, and in [MOW98] it is shown that call-by-name and call-by-need evaluation induce the same observational equivalences for a call-by-need lambda calculus with non-recursive let. Additionally, the extension of the corresponding calculi by recursive let is discussed in [AFM ${ }^{+} 95$, AF97], and further call-by-need lambda calculi with a recursive let are presented in $\mathrm{AB} 97, \mathrm{AB} 02, \mathrm{NH} 09$ where [NH09] study the equivalence between a natural semantics and a reductions semantics. In AB02 it is shown that there exist infinite normal forms and that the calculus satisfies a form of confluence. All these calculi correspond to our calculus $L_{L R}$. A difference is that the let-shifting in the standard reduction in the mentioned works is different from $L_{L R}$. However, this difference is not substantial, since it does not influence the contextual semantics. A more substantial difference is that $L_{L R}$ combines recursive let with data constructors, case-expressions and seq, which none of the related works do.

In [MS99] a call-by-need calculus is analyzed which is closer to our calculus $L_{L R}$, since letrec, case, and constructors are present (but not seq). Another difference is that [MS99] uses an abstract machine semantics as operational semantics, while their approach to program equivalence is based on contextual equivalence, as is ours.

The operational semantics of call-by-need lambda calculi with letrec are investigated in [Lau93] and [Ses97, where the former proposed a natural semantics, and proved it correct and adequate with respect to a denotational semantics, and the latter derived an efficient abstract machine from the natural semantics.

Investigations of the semantics of lazy functional programming languages including the seq-operator can be found in [JV06, VJ07.

Outline. In Sect. 2 we introduce some common notions of program calculi, contextual equivalence, similarity and also of translations between those calculi. In Sect. 3 we introduce the extension $L_{l c c}$ of Abramsky's lazy lambda calculus with case, constructors, and seq, and two letrec-calculi $L_{L R}, L_{\text {name }}$ as further syntactic extensions. In Sect. 4 we show that for so-called "convergence admissible" calculi an alternative inductive characterization of similarity is possible. We then use Howe's method in $L_{l c c}$ to show that contextual approximation and a standard version of applicative similarity coincide. Proving that $L_{l c c}$ is convergence admissible then implies that the alternative inductive characterization of similarity can be used for $L_{l c c}$. In Sect. 5 and 6 the translations $W$ and $N$ are introduced and the full-abstraction results are obtained. In Sect. 7 we show soundness and completeness of our variants of applicative similarity w.r.t. contextual equivalence in $L_{L R}$. We conclude in Sect. 8. 


\section{Common Notions and Notations for CAlculi}

Before we explain the specific calculi, some common notions are introduced. A calculus definition consists of its syntax together with its operational semantics which defines the evaluation of programs and the implied equivalence of expressions:

Definition 2.1. An untyped deterministic calculus $D$ is a four-tuple $(\mathbb{E}, \mathbb{C}, \rightarrow, \mathbb{A})$, where $\mathbb{E}$ are expressions (up to $\alpha$-equivalence), $\mathbb{C}: \mathbb{E} \rightarrow \mathbb{E}$ is a set of functions (which usually represents contexts), $\rightarrow$ is a small-step reduction relation (usually the normal-order reduction), which is a partial function on expressions (i.e., deterministic), and $\mathbb{A} \subset \mathbb{E}$ is a set of answers of the calculus.

For $C \in \mathbb{C}$ and an expression $s$, the functional application is denoted as $C[s]$. For contexts, this is the replacement of the hole of $C$ by $s$. We also assume that the identity function $I d$ is contained in $\mathbb{C}$ with $I d[s]=s$ for all expressions $s$, and that $\mathbb{C}$ is closed under composition, i.e. $C_{1}, C_{2} \in \mathbb{C} \Longrightarrow C_{1} \circ C_{2} \in \mathbb{C}$.

The transitive closure of $\rightarrow$ is denoted as $\stackrel{+}{\rightarrow}$ and the transitive and reflexive closure of $\rightarrow$ is denoted as $\stackrel{*}{\rightarrow}$. The notation $\stackrel{0 \vee 1}{\longrightarrow}$ means equality or one reduction, and $\stackrel{k}{\rightarrow}$ means $k$ reductions. Given an expression $s$, a sequence $s \rightarrow s_{1} \rightarrow \ldots \rightarrow s_{n}$ is called a reduction sequence; it is called an evaluation if $s_{n}$ is an answer, i.e. $s_{n} \in \mathbb{A}$; in this case we say $s$ converges and denote this as $s \downarrow_{D} s_{n}$ or as $s \downarrow_{D}$ if $s_{n}$ is not important. If there is no $s_{n}$ s.t. $s \downarrow_{D} s_{n}$ then $s$ diverges, denoted as $s \Uparrow_{D}$. When dealing with multiple calculi, we often use the calculus name to mark its expressions and relations, e.g. $\stackrel{D}{\rightarrow}$ denotes a reduction relation in $D$.

We will have to deal with several calculi and preorders. Throughout this paper we will use the symbol $\preccurlyeq$ for co-inductively defined preorders (i.e. similarities), and $\leq$ for (inductively defined or otherwise defined) contextual preorders. For the corresponding symmetrizations we use $\simeq$ for $\preccurlyeq \cap \succcurlyeq$ and $\sim$ for $\leq \cap \geq$. All the symbols are always indexed by the corresponding calculus and sometimes more restrictions like specific sets of contexts are attached to the indices of the symbols.

Contextual approximation and equivalence can be defined in a general way:

Definition 2.2 (Contextual Approximation and Equivalence, $\leq_{D}$ and $\sim_{D}$ ). Let $D=$ $\left(\mathbb{E}, \mathbb{C}, \rightarrow, \mathbb{A}\right.$ ) be a calculus and $s_{1}, s_{2}$ be $D$-expressions. Contextual approximation (or contextual preorder $) \leq_{D}$ and contextual equivalence $\sim_{D}$ are defined as:

$$
\begin{array}{lll}
s_{1} \leq_{D} s_{2} & \text { iff } & \forall C \in \mathbb{C}: C\left[s_{1}\right] \downarrow_{D} \Rightarrow C\left[s_{2}\right] \downarrow_{D} \\
s_{1} \sim \sim_{D} s_{2} & \text { iff } \quad s_{1} \leq_{D} s_{2} \wedge s_{2} \leq_{D} s_{1}
\end{array}
$$

A program transformation is a binary relation $\eta \subseteq(\mathbb{E} \times \mathbb{E})$. A program transformation $\eta$ is called correct iff $\eta \subseteq \sim_{D}$.

Note that $\leq_{D}$ is a precongruence, i.e., $\leq_{D}$ is reflexive, transitive, and $s \leq_{D} t$ implies $C[s] \leq_{D} C[t]$ for all $C \in \mathbb{C}$, and that $\sim_{D}$ is a congruence, i.e. a precongruence and an equivalence relation.

We also define a general notion of similarity coinductively for untyped deterministic calculi. We first define the operator $F_{D, \mathcal{Q}}$ on binary relations of expressions:

Definition 2.3. Let $D=(\mathbb{E}, \mathbb{C}, \rightarrow, \mathbb{A})$ be an untyped deterministic calculus and let $\mathcal{Q} \subseteq \mathbb{C}$ be a set of functions on expressions (i.e. $\forall Q \in \mathcal{Q}: Q: \mathbb{E} \rightarrow \mathbb{E}$ ). Then the $\mathcal{Q}$-experiment 
operator $F_{D, \mathcal{Q}}: 2^{(\mathbb{E} \times \mathbb{E})} \rightarrow 2^{(\mathbb{E} \times \mathbb{E})}$ is defined as follows for $\eta \subseteq \mathbb{E} \times \mathbb{E}$ :

$$
s_{1} F_{D, \mathcal{Q}}(\eta) s_{2} \text { iff } s_{1} \downarrow_{D} v_{1} \Longrightarrow \exists v_{2} \cdot\left(s_{2} \downarrow_{2} \wedge \forall Q \in \mathcal{Q}: Q\left(v_{1}\right) \eta Q\left(v_{2}\right)\right)
$$

Lemma 2.4. The operator $F_{D, \mathcal{Q}}$ is monotonous w.r.t. set inclusion, i.e. for all binary relations $\eta_{1}, \eta_{2}$ on expressions $\eta_{1} \subseteq \eta_{2} \Longrightarrow F_{D, \mathcal{Q}}\left(\eta_{1}\right) \subseteq F_{D, \mathcal{Q}}\left(\eta_{2}\right)$.

Proof. Let $\eta_{1} \subseteq \eta_{2}$ and $s_{1} F_{D, \mathcal{Q}}\left(\eta_{1}\right) s_{2}$. From the assumption $s_{1} F_{D, \mathcal{Q}}\left(\eta_{1}\right) s_{2}$ the implication $s_{1} \downarrow_{D} v_{1} \Longrightarrow\left(s_{2} \downarrow_{D} v_{2} \wedge \forall Q \in \mathcal{Q}: Q\left(v_{1}\right) \eta_{1} Q\left(v_{2}\right)\right)$ follows. From $\eta_{1} \subseteq \eta_{2}$ the implication $s_{1} \downarrow v_{1} \Longrightarrow\left(s_{2} \downarrow_{D} v_{2} \wedge \forall Q \in \mathcal{Q}: Q\left(v_{1}\right) \eta_{2} Q\left(v_{2}\right)\right)$ follows. Thus, $s_{1} F_{\mathcal{Q}}\left(\eta_{2}\right) s_{2}$.

Since $F_{D, \mathcal{Q}}$ is monotonous, its greatest fixpoint exists:

Definition 2.5 ( $\mathcal{Q}$-Similarity, $\preccurlyeq_{D, \mathcal{Q}}$ ). The behavioral preorder $\preccurlyeq_{D, \mathcal{Q}}$, called $\mathcal{Q}$-similarity, is defined as the greatest fixed point of $F_{D, \mathcal{Q}}$.

We also provide an inductive definition of behavioral equivalence, which is defined as a contextual preorder where the contexts are restricted to the set $\mathcal{Q}$ (and the empty context).

Definition 2.6. Let $D=(\mathbb{E}, \mathbb{C}, \rightarrow, \mathbb{A})$ be an untyped deterministic calculus, and $\mathcal{Q} \subseteq \mathbb{C}$. Then the relation $\leq_{D, \mathcal{Q}}$ is defined as follows:

$$
s_{1} \leq{ }_{D, \mathcal{Q}} s_{2} \text { iff } \forall n \geq 0: \forall Q_{i} \in \mathcal{Q}: Q_{1}\left(Q_{2}\left(\ldots\left(Q_{n}\left(s_{1}\right)\right)\right)\right) \downarrow_{D} \Longrightarrow Q_{1}\left(Q_{2}\left(\ldots\left(Q_{n}\left(s_{2}\right)\right)\right)\right) \downarrow_{D}
$$

Note that contextual approximation is a special case of this definition, i.e. $\leq_{D}=\leq_{D, \mathbb{C}}$.

Later in Section 4.1 we will provide a sufficient criterion on untyped deterministic calculi that ensures that $\preccurlyeq_{D, \mathcal{Q}}$ and $\leq_{D, \mathcal{Q}}$ coincide.

We are interested in translations between calculi that are faithful w.r.t. the corresponding contextual preorders.

Definition 2.7 ([SSNSS08, SSNSS09]). For $i=1,2$ let $\left(\mathbb{E}_{i}, \mathbb{C}_{i}, \rightarrow_{i}, \mathbb{A}_{i}\right)$ be untyped deterministic calculi. A translation $\tau:\left(\mathbb{E}_{1}, \mathbb{C}_{1}, \rightarrow_{1}, \mathbb{A}_{1}\right) \rightarrow\left(\mathbb{E}_{2}, \mathbb{C}_{2}, \rightarrow_{2}, \mathbb{A}_{2}\right)$ is a mapping $\tau_{E}: \mathbb{E}_{1} \rightarrow \mathbb{E}_{2}$ and a mapping $\tau_{C}: \mathbb{C}_{1} \rightarrow \mathbb{C}_{2}$ such that $\tau_{C}\left(I d_{1}\right)=I d_{2}$. The following properties of translations are defined:

- $\tau$ is compositional iff $\tau(C[s])=\tau(C)[\tau(s)]$ for all $C, s$.

- $\tau$ is convergence equivalent iff $s \downarrow_{1} \Longleftrightarrow \tau(s) \downarrow_{2}$ for all $s$.

- $\tau$ is adequate iff for all $s, t \in \mathbb{E}_{1}: \tau(s) \leq_{2} \tau(t) \Longrightarrow s \leq_{1} t$.

- $\tau$ is fully abstract iff for all $s, t \in \mathbb{E}_{1}: s \leq_{1} t \Longleftrightarrow \tau(s) \leq_{2} \tau(t)$.

- $\tau$ is an isomorphism iff it is fully abstract and a bijection on the quotients $\tau / \sim: \mathbb{E}_{1} / \sim \rightarrow \mathbb{E}_{2} / \sim$.

Note that isomorphism means an order-isomorphism between the term-models, where the orders are $\leq_{1} / \sim$ and $\leq_{2} / \sim$ (which are the relations in the quotient).

Proposition 2.8 ([SSNSS08, SSNSS09]). Let $\left(\mathbb{E}_{i}, \mathbb{C}_{i}, \rightarrow_{i}, \mathbb{A}_{1}\right)$ for $i=1,2$ be untyped deterministic calculi. If a translation $\tau:\left(\mathbb{E}_{1}, \mathbb{C}_{1}, \rightarrow_{1}, \mathbb{A}_{1}\right) \rightarrow\left(\mathbb{E}_{2}, \mathbb{C}_{2}, \rightarrow_{2}, \mathbb{A}_{2}\right)$ is compositional and convergence equivalent, then it is also adequate.

Proof. Let $s, t \in \mathbb{E}_{1}$ with $\tau(s) \leq_{2} \tau(t)$ and let $C[s] \downarrow_{1}$ for some $C \in \mathbb{C}$. It is sufficient to show that this implies $C[t] \downarrow_{1}$ : Convergence equivalence shows that $\tau(C[s]) \downarrow_{2}$. Compositionality implies $\tau(C)[\tau(s)] \downarrow_{2}$, and then $\tau(s) \leq_{2} \tau(t)$ implies $\tau(C)[\tau(t)] \downarrow_{2}$. Compositionality applied once more implies $\tau(C[t]) \downarrow_{2}$, and then convergence equivalence finally implies $C[t] \downarrow_{1}$. 


\section{Three Calculi}

In this section we introduce the calculi $L_{L R}, L_{n a m e}$, and $L_{l c c} . L_{L R}$ is a call-by-need calculus with recursive let, data constructors, case-expressions, and the seq-operator. The calculus $L_{\text {name }}$ has the same syntactic constructs as $L_{L R}$, but uses a call-by-name, rather than a callby-need, evaluation. The calculus $L_{l c c}$ does not have letrec, and also uses a call-by-name evaluation.

For all three calculi we assume that there is a (common) set of data constructors $c$ which is partitioned into types, such that every constructor $c$ belongs to exactly one type. We assume that for every type $T$ the set of its corresponding data constructors can be enumerated as $c_{T, 1}, \ldots, c_{T,|T|}$ where $|T|$ is the number of data constructors of type $T$. We also assume that every constructor has a fixed arity denoted as $\operatorname{ar}(c)$ which is a non-negative integer. We assume that there is a type Bool among the types, with the data constructors False and True both of arity 0 . We require that data constructors occur only fully saturated, i.e. a constructor $c$ is only allowed to occur together with $\operatorname{ar}(c)$ arguments, written as $\left(\begin{array}{llll}c & s_{1} & \ldots & s_{\operatorname{ar}(c)}\end{array}\right)$ where $s_{i}$ are expressions of the corresponding calculus 1 . We also write $(c \vec{s})$ as an abbreviation for the constructor application $\left(\begin{array}{ccccc}c & s_{1} & \ldots & \left.s_{\operatorname{ar}(c)}\right)\end{array}\right)$. All three calculi allow deconstruction via case-expressions:

$$
\operatorname{case}_{T} s \text { of }\left(c_{T, 1} x_{1,1} \ldots x_{1, \operatorname{ar}\left(c_{T, 1}\right)} \rightarrow s_{1}\right) \ldots\left(c_{T,|T|} x_{|T|, 1} \ldots x_{|T|, \operatorname{ar}\left(c_{T,|T|}\right)} \rightarrow s_{|T|}\right)
$$

where $s, s_{i}$ are expressions and $x_{i, j}$ are variables of the corresponding calculus. Thus there is

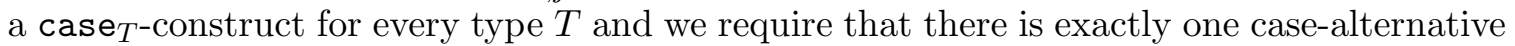
$\left(\begin{array}{llll}c_{T, i} & x_{i, 1} & \ldots & x_{i, \operatorname{ar}\left(c_{T, i}\right)} \rightarrow s_{i}\end{array}\right)$ for every constructor $c_{T, i}$ of type $T$. In a case-alternative $\left(c_{T, i} x_{i, 1} \ldots x_{i, \operatorname{ar}\left(c_{T, i}\right)} \rightarrow s_{i}\right)$ we call $c_{T, i} x_{i, 1} \ldots x_{i, \operatorname{ar}\left(c_{T, i}\right)}$ a pattern and $s_{i}$ the right hand side of the alternative. All variables in a case-pattern must be pairwise distinct. We will sometimes abbreviate the case-alternatives by alts if the exact terms of the alternatives are not of interest. As a further abbreviation we sometimes write if $s_{1}$ then $s_{2}$ else $s_{3}$ for the case-expression ( case $_{\text {Bool }} s_{1}$ of (True $\rightarrow s_{2}$ ) (False $\rightarrow s_{3}$ )).

We now define the syntax of expressions with letrec, i.e. the set $\mathbb{E}_{\mathcal{L}}$ of expressions which are used in both of the calculi $L_{L R}$ and $L_{\text {name }}$.

Definition 3.1 (Expressions $\mathbb{E}_{\mathcal{L}}$ ). The set $\mathbb{E}_{\mathcal{L}}$ of expressions is defined by the following grammar, where $x, x_{i}$ are variables:

$$
\begin{aligned}
r, s, t, r_{i}, s_{i}, t_{i} \in \mathbb{E}_{\mathcal{L}}::= & x|(s t)|(\lambda x . s) \mid\left(\text { letrec } x_{1}=s_{1}, \ldots, x_{n}=s_{n} \text { in } t\right) \\
& \left|\left(c s_{1} \ldots s_{\operatorname{ar}(c)}\right)\right|(\operatorname{seq} s t) \mid\left(\operatorname{case}_{T} s \text { of alts }\right)
\end{aligned}
$$

We assign the names application, abstraction, seq-expression, or letrec-expression to the expressions $(s t),(\lambda x . s)$, ( seq $s t)$, or (letrec $x_{1}=s_{1}, \ldots, x_{n}=s_{n}$ in $t$ ), respectively. A value $v$ is defined as an abstraction or a constructor application. A group of letrec bindings is sometimes abbreviated as Env. We use the notation $\left\{x_{g(i)}=s_{h(i)}\right\}_{i=m}^{n}$ for the chain $x_{g(m)}=s_{h(m)}, x_{g(m+1)}=s_{h(m+1)}, \ldots, x_{g(n)}=s_{h(n)}$ of bindings where $g, h: \mathbb{N} \rightarrow \mathbb{N}$ are injective, e.g., $\left\{x_{i}=s_{i-1}\right\}_{i=m}^{n}$ means the bindings $x_{m}=s_{m-1}, x_{m+1}=s_{m}, \ldots x_{n}=s_{n-1}$. We assume that variables $x_{i}$ in letrec-bindings are all distinct, that letrec-expressions are identified up to reordering of binding-components, and that, for convenience, there is at least one binding. letrec-bindings are recursive, i.e., the scope of $x_{j}$ in (letrec $x_{1}=$ $s_{1}, \ldots, x_{n-1}=s_{n-1}$ in $\left.s_{n}\right)$ are all expressions $s_{i}$ with $1 \leq i \leq n$.

\footnotetext{
${ }^{1}$ Partial applications of constructors of the form $c s_{1} \ldots s_{n}$ (as e.g. available in Haskell) thus have to be represented by $\lambda x_{n+1} \ldots \lambda x_{\operatorname{ar}(c)} . c s_{1} \ldots s_{n} x_{n+1} \ldots x_{\operatorname{ar}(c)}$.
} 
$\mathbb{C}_{\mathcal{L}}$ denotes the set of all contexts for the expressions $\mathbb{E}_{\mathcal{L}}$.

Free and bound variables in expressions and $\alpha$-renamings are defined as usual. The set of free variables in $s$ is denoted as $F V(s)$.

Convention 3.2 (Distinct Variable Convention). We use the distinct variable convention, i.e., all bound variables in expressions are assumed to be distinct, and free variables are distinct from bound variables. All reduction rules are assumed to implicitly $\alpha$-rename bound variables in the result if necessary.

In all three calculi we will use the symbol $\Omega$ for the specific (letrec-free) expression $(\lambda z .(z z))(\lambda x .(x x))$. In all of our calculi $\Omega$ is divergent and the least element of the corresponding contextual preorder. This is proven in SSSS08] for $L_{L R}$ and can easily be proven for the other two calculi using standard methods, such as context lemmas. Note that this property also follows from the Main Theorem 7.6 for all three calculi.

3.1. The Call-by-Need Calculus $L_{L R}$. We begin with the call-by-need lambda calculus $L_{L R}$ which is exactly the call-by-need calculus of [SSSS08. It has a rather complex form of reduction rules using variable chains. The justification is that this formulation permits direct syntactic proofs of correctness w.r.t. contextual equivalence for a large class of transformations. Several modifications of the reduction strategy, removing indirections, do not change the semantics of the calculus, however, they appear to be not treatable by syntactic proof methods using diagrams (see [SSSS08]). $L_{L R}$-expressions are exactly the expressions $\mathbb{E}_{\mathcal{L}}$.

Definition 3.3. The reduction rules for the calculus and language $L_{L R}$ are defined in Fig. 2, where the labels $S, V$ are used for the exact definition of the normal-order reduction below. Several reduction rules are denoted by their name prefix: the union of (llet-in) and (llet-e) is called (llet). The union of (llet), (lapp), (lcase), and (lseq) is called (lll).

For the definition of the normal order reduction strategy of the calculus $L_{L R}$ we use the labeling algorithm in Fig. 3 which detects the position where a reduction rule is applied according to the normal order. It uses the following labels: $S$ (subterm), $T$ (top term), $V$ (visited), and $W$ (visited, but not target). We use $\vee$ when a rule allows two options for a label, e.g. $s^{S \vee T}$ stands for $s$ labeled with $S$ or $T$.

A labeling rule $l \leadsto r$ is applicable to a (labeled) expression $s$ if $s$ matches $l$ with the labels given by $l$, where $s$ may have more labels than $l$ if not otherwise stated. The labeling algorithm takes an expression $s$ as its input and exhaustively applies the rules in Fig. 3 to $s^{T}$, where no other subexpression in $s$ is labeled. The label $T$ is used to prevent the labeling algorithm from descending into letrec-environments that are not at the top of the expression. The labels $V$ and $W$ mark the visited bindings of a chain of bindings, where $W$ is used for variable-to-variable bindings. The labeling algorithm either terminates with fail or with success, where in general the direct superterm of the $S$-marked subexpression indicates a potential normal-order redex. The use of such a labeling algorithm corresponds to the search of a redex in term graphs where it is usually called unwinding.

Definition 3.4 (Normal Order Reduction of $L_{L R}$ ). Let $s$ be an expression. Then a single normal order reduction step $\stackrel{L R}{\longrightarrow}$ is defined as follows: first the labeling algorithm in Fig. 3 is applied to $s$. If the labeling algorithm terminates successfully, then one of the rules in Fig. 2 is applied, if possible, where the labels $S, V$ must match the labels in the expression 


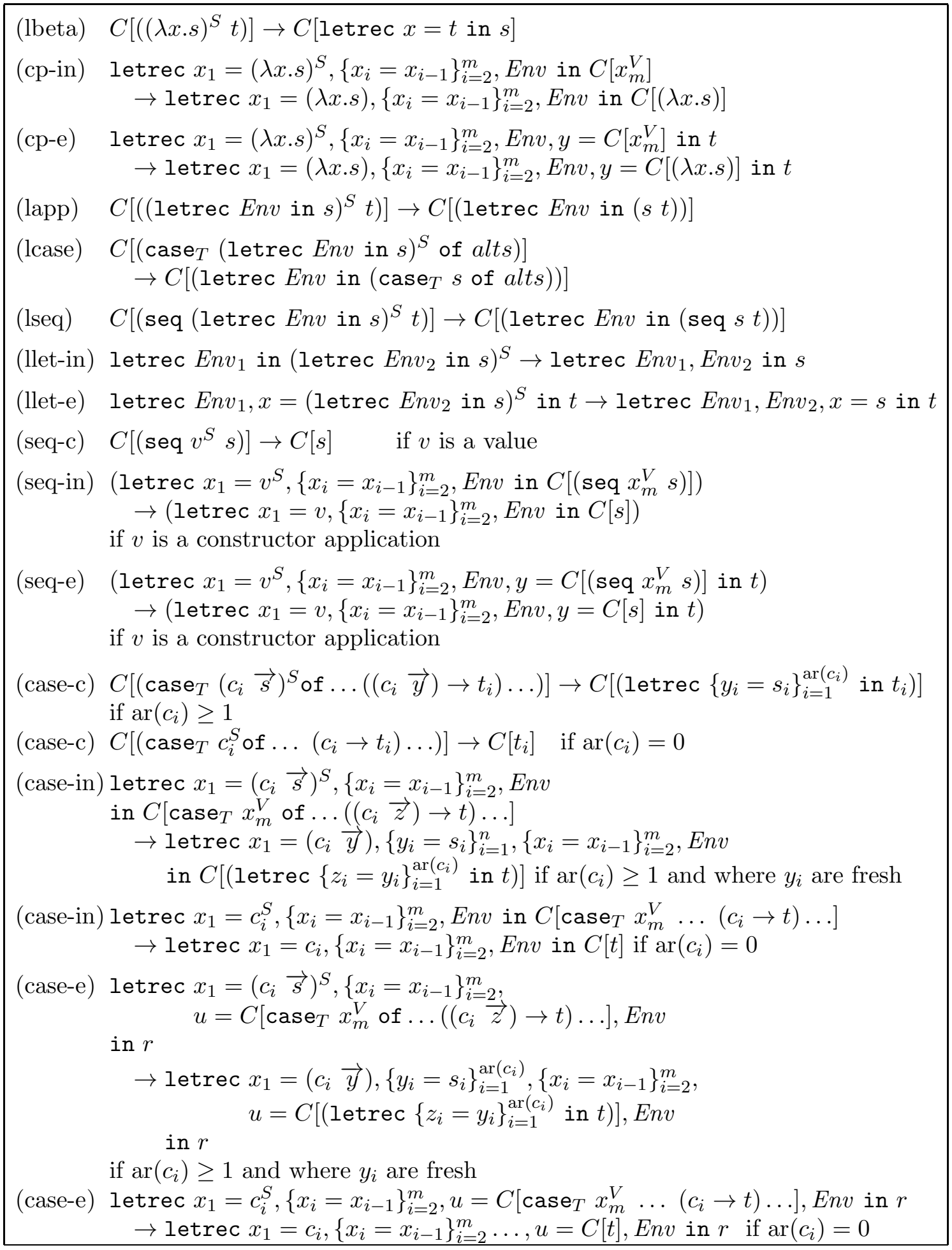

Figure 2: Reduction rules of $L_{L R}$ 


\begin{tabular}{|c|c|}
\hline $\begin{array}{l}(\text { letrec Env in } s)^{T} \\
(s t)^{S \vee T} \\
(\text { seq } s t)^{S \vee T} \\
\left(\text { case }_{T} s \text { of alts) } S \vee T\right. \\
\left(\text { letrec } x=s, \text { Env in } C\left[x^{S}\right]\right) \\
\left(\text { letrec } x=s^{V \vee W}, y=C\left[x^{S}\right], \text { Env in } t\right) \\
\left(\text { letrec } x=C\left[x^{S}\right], \text { Env in } s\right) \\
\left(\text { letrec } x=s, y=C\left[x^{S}\right], \text { Env in } t\right) \\
\left(\text { letrec } x=s, y=x^{S}, \text { Env in } t\right)\end{array}$ & $\begin{array}{l}\leadsto\left(\text { letrec Env in } s^{S}\right)^{V} \\
\leadsto\left(s^{S} t\right)^{V} \\
\leadsto\left(\text { seq } s^{S} t\right)^{V} \\
\leadsto\left(\operatorname{case}_{T} s^{S} \text { of alts }\right)^{V} \\
\leadsto\left(\text { letrec } x=s^{S}, \text { Env in } C\left[x^{V}\right]\right) \\
\leadsto \text { fail } \\
\leadsto \text { fail } \\
\leadsto\left(\text { letrec } x=s^{S}, y=C\left[x^{V}\right], \text { Env in } t\right) \\
\\
\quad \text { if } C[x] \neq x \\
\left(\text { letrec } x=s^{S}, y=x^{W}, \text { Env in } t\right)\end{array}$ \\
\hline
\end{tabular}

Figure 3: Labeling algorithm for $L_{L R}$

$s$ (again $s$ may have more labels). The normal order redex is defined as the left-hand side of the applied reduction rule. The notation for a normal-order reduction that applies the rule $a$ is $\stackrel{L R, a}{\longrightarrow}$, e.g. $\stackrel{L R, \text { lapp }}{\longrightarrow}$ applies the rule (lapp).

The normal order reduction of $L_{L R}$ implements a call-by-need reduction with sharing which avoids substitution of arbitrary expressions. We describe the rules: The rule (lbeta) is a sharing variant of classical $\beta$-reduction, where the argument of an abstraction is shared by a new letrec-binding instead of substituting the argument in the body of an abstraction. The rules (cp-in) and (cp-e) allow to copy abstractions into needed positions. The rules (lapp), (lcase), and (lseq) allow moving letrec-expressions to the top of the term if they are inside a reduction position of an application, a case-expression, or a seq-expression. To flatten nested letrec-expressions, the rules (llet-in) and (llet-e) are added to the reduction. Evaluation of seq-expressions is performed by the rules (seq-c), (seq-in), and (seq-e), where the first argument of seq must be a value (rule seq-c) or it must be a variable which is bound in the outer letrec-environment to a constructor application. Since normal order reduction avoids copying constructor applications, the rules (seq-in) and (seq-e) are required. Correspondingly, the evaluation of case-expressions requires several variants: there are again three rules for the cases where the argument of case is already a constructor application (rule (case-c)) or where the argument is a variable which is bound to a constructor application (perhaps by several indirections in the letrec-environment) which are covered by the rule (case-in) and (case-e). All three rules have two variants: one variant for the case when a constant is scrutinized (and thus no arguments need to be shared by new letrec-bindings) and another variant for the case when arguments are present (and thus the arity of the scrutinized constructor is strictly greater than 0 ). For the latter case the arguments of the constructor application are shared by new letrec-bindings, such that the newly created variables can be used as references in the right hand side of the matching alternative.

Definition 3.5. A reduction context $R_{L R}$ is any context, such that its hole is labeled with $S$ or $T$ by the $L_{L R}$-labeling algorithm.

Of course, reduction contexts could also be defined recursively, as in SSSS08, Definition 1.5], but such a definition is very cumbersome due to a large number of special cases. The labeling algorithm provides a definition that, in our experience, is easier to work with. 


(gc) $C\left[\right.$ letrec $\left\{x_{i}=s_{i}\right\}_{i=1}^{n}$ in $\left.t\right] \rightarrow C[t]$, if $F V(t) \cap\left\{x_{1}, \ldots, x_{n}\right\}=\emptyset$
(gc) $C\left[\right.$ letrec $\left\{x_{i}=s_{i}\right\}_{i=1}^{n},\left\{y_{i}=t_{i}\right\}_{i=1}^{m}$ in $\left.t\right] \rightarrow C\left[\operatorname{letrec}\left\{y_{i}=t_{i}\right\}_{i=1}^{m}\right.$ in $\left.t\right]$,
if $\left(F V(t) \cup \bigcup_{i=1}^{m} F V\left(t_{i}\right)\right) \cap\left\{x_{1}, \ldots, x_{n}\right\}=\emptyset$
$($ lwas) $C[(s$ (letrec Env in $t))] \rightarrow C[$ letrec $E n v$ in $(s t)]$
$\left(\right.$ lwas) $C\left[\left(c s_{1} \ldots\left(\right.\right.\right.$ letrec Env in $\left.\left.\left.s_{i}\right) \ldots s_{n}\right)\right] \rightarrow C\left[\right.$ letrec Env in $\left.\left(c s_{1} \ldots s_{i} \ldots s_{n}\right)\right]$
$($ lwas) $C[($ seq $s($ letrec Env in $t))] \rightarrow C[$ letrec Env in seq $s t]$

Figure 4: Transformations for garbage collection and letrec-shifting

By induction on the term structure one can easily verify that the normal order redex, as well as the normal order reduction, is unique. A weak head normal form in $L_{L R}\left(L_{L R^{-}}\right.$

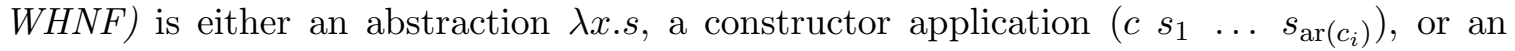
expression (letrec Env in $v$ ) where $v$ is a constructor application or an abstraction, or an expression of the form (letrec $x_{1}=v,\left\{x_{i}=x_{i-1}\right\}_{i=2}^{m}$, Env in $x_{m}$ ), where $v=(c \vec{s})$. We distinguish abstraction-WHNF (AWHNF) and constructor WHNF (CWHNF) based on whether the value $v$ is an abstraction or a constructor application, respectively. The notions of convergence, divergence and contextual approximation are as defined in Sect. 2, If there is no normal order reduction originating at an expression $s$ then $s \Uparrow_{L R}$. This, in particular, means that expressions for which the labeling algorithm fails to find a redex, or for which there is no matching constructor for a subexpression (that is a WHNF) in a case redex position, or expressions with cyclic dependencies like letrec $x=x$ in $x$, are diverging.

Example 3.6. We consider the expression $s_{1}:=\operatorname{letrec} x=(y \lambda u . u), y=\lambda z . z$ in $x$. The labeling algorithm applied to $s_{1}$ yields (letrec $x=\left(y^{V} \lambda u . u\right)^{V}, y=(\lambda z . z)^{S}$ in $\left.x^{V}\right)^{V}$. The reduction rule that matches this labeling is the reduction rule (cp-e), i.e. $s_{1} \stackrel{L R}{\longrightarrow}$ (letrec $x=\left(\left(\lambda z^{\prime} \cdot z^{\prime}\right) \lambda u . u\right), y=(\lambda z . z)$ in $\left.x\right)=s_{2}$. The labeling of $s_{2}$ is (letrec $x=$ $\left(\left(\lambda z^{\prime} . z^{\prime}\right)^{S} \lambda u . u\right)^{V}, y=(\lambda z . z)$ in $\left.x^{V}\right)^{V}$, which makes the rule (lbeta) applicable, i.e. $s_{2} \stackrel{L R}{\longrightarrow}$ (letrec $x=\left(\right.$ letrec $z^{\prime}=\lambda u$.u in $\left.z^{\prime}\right), y=(\lambda z . z)$ in $\left.x\right)=s_{3}$. The labeling of $s_{3}$ is (letrec $x=\left(\text { letrec } z^{\prime}=\lambda u . u \text { in } z^{\prime}\right)^{S}, y=(\lambda z . z)$ in $\left.x^{V}\right)^{V}$. Thus an (llet-e)-reduction is applicable to $s_{3}$, i.e. $s_{3} \stackrel{L R}{\longrightarrow}\left(\operatorname{letrec} x=z^{\prime}, z^{\prime}=\lambda u . u, y=(\lambda z . z)\right.$ in $\left.x\right)=s_{4}$. Now $s_{4}$ gets labeled as (letrec $x=z^{\prime W}, z^{\prime}=(\lambda u . u)^{S}, y=(\lambda z . z)$ in $\left.x^{V}\right)^{V}$, and a (cp-in)-reduction is applicable, i.e. $s_{4} \stackrel{L R}{\longrightarrow}\left(\right.$ letrec $x=z^{\prime}, z^{\prime}=(\lambda u . u), y=(\lambda z . z)$ in $\left.(\lambda u . u)\right)=s_{5}$. The labeling algorithm applied to $s_{5}$ yields (letrec $x=z^{\prime}, z^{\prime}=(\lambda u . u), y=(\lambda z . z)$ in $\left.(\lambda u . u)^{S}\right)^{V}$, but no reduction is applicable to $s_{5}$, since $s_{5}$ is a WHNF.

Concluding, the calculus $L_{L R}$ is defined by the tuple $\left(\mathbb{E}_{\mathcal{L}}, \mathbb{C}_{\mathcal{L}}, \stackrel{L R}{\longrightarrow}, \mathbb{A}_{L R}\right)$ where $\mathbb{A}_{L R}$ are the $L_{L R}$-WHNFs, where we equate alpha-equivalent expressions, contexts and answers.

In [SSSS08] correctness of several program transformations was shown:

Theorem 3.7 ([SSSS08, Theorems 2.4 and 2.9]). All reduction rules shown in Fig. 20 are correct program transformations, even if they are used with an arbitrary context $C$ in the rules without requiring the labels. The transformations for garbage collection (gc) and for shifting of letrec-expressions (lwas) shown in Fig. 因are also correct program transformations. 


$\begin{aligned}(\text { letrec Env in } s)^{X} & \leadsto\left(\text { letrec Env in } s^{X}\right) \text { if } X \text { is } S \text { or } T \\ (s t)^{S \vee T} & \leadsto\left(s^{S} t\right) \\ (\operatorname{seq} t)^{S \vee T} & \leadsto\left(\operatorname{seq}^{S} t\right) \\ \left(\operatorname{case}_{T} s \text { of alts }\right)^{S \vee T} & \leadsto\left(\operatorname{case}_{T} s^{S} \text { of alts }\right)\end{aligned}$

Figure 5: Labeling algorithm for $L_{\text {name }}$

\begin{tabular}{|c|c|}
\hline (beta) & $C\left[(\lambda x . s)^{S} t\right] \rightarrow C[s[t / x]]$ \\
\hline$(\mathrm{gcp})$ & $C_{1}\left[\right.$ letrec Env, $x=s$ in $\left.C_{2}\left[x^{S \vee T}\right]\right] \rightarrow C_{1}\left[\right.$ letrec Env, $x=s$ in $\left.C_{2}[s]\right]$ \\
\hline (lapp) & $C\left[\left((\text { letrec Env in } s)^{S} t\right)\right] \rightarrow C[($ letrec Env in $(s t))]$ \\
\hline (lcase) & $\begin{array}{l}C\left[\left(\text { case }_{T}(\text { letrec Env in } s)^{S} \text { of alts }\right)\right] \\
\quad \rightarrow C\left[\left(\text { letrec Env in }\left(\operatorname{case}_{T} s \text { of alts }\right)\right)\right]\end{array}$ \\
\hline (lseq) & $C\left[\left(\operatorname{seq}(\text { letrec Env in } s)^{S} t\right)\right] \rightarrow C[($ letrec Env in $($ seq $s t))]$ \\
\hline (seq-c) & $C\left[\left(\operatorname{seq} v^{S} s\right)\right] \rightarrow C[s] \quad$ if $v$ is a value \\
\hline (case) & $\begin{array}{l}C\left[\left(\operatorname{case}_{T}\left(c s_{1} \ldots s_{\operatorname{ar}(c)}\right)^{S} \text { of } \ldots\left(\left(c x_{1} \ldots x_{\operatorname{ar}(c)}\right) \rightarrow t\right) \ldots\right)\right] \\
\quad \rightarrow C\left[t\left[s_{1} / x_{1}, \ldots, s_{\operatorname{ar}(c)} / x_{\operatorname{ar}(c)}\right]\right]\end{array}$ \\
\hline
\end{tabular}

Figure 6: Normal order reduction rules $\stackrel{\text { name }}{\longrightarrow}$ of $L_{\text {name }}$

3.2. The Call-by-Name Calculus $L_{\text {name }}$. Now we define a call-by-name calculus on $\mathbb{E}_{\mathcal{L}^{-}}$ expressions. The calculus $L_{\text {name }}$ has $\mathbb{E}_{\mathcal{L}}$ as expressions, but the reduction rules are different from $L_{L R}$. The calculus $L_{\text {name }}$ does not implement a sharing strategy but instead performs the usual call-by-name beta-reduction and copies arbitrary expressions directly into needed positions.

In Fig. 5 the rules of the labeling algorithm for $L_{n a m e}$ are given. The algorithm uses the labels $S$ and $T$. For an expression $s$ the labeling starts with $s^{T}$.

An $L_{\text {name }}$ reduction context $R_{\text {name }}$ is any context where the hole is labeled $T$ or $S$ by the labeling algorithm, or more formally they can be defined as follows:

Definition 3.8. Reduction contexts $R_{\text {name }}$ are contexts of the form $L[A]$ where the context classes $\mathcal{A}$ and $\mathcal{L}$ are defined by the following grammar, where $s$ is any expression:

$$
\begin{aligned}
& L \in \mathcal{L}::=[\cdot] \mid \text { letrec Env in } L \\
& A \in \mathcal{A}::=[\cdot]|(A s)|\left(\operatorname{case}_{T} A \text { of alts }\right) \mid(\operatorname{seq} A s)
\end{aligned}
$$

Normal order reduction $\stackrel{\text { name }}{\longrightarrow}$ of $L_{\text {name }}$ is defined by the rules shown in Fig. [6 where the labeling algorithm according to Fig. 5 must be applied first. Note that the rules (seq-c), (lapp), (lcase), and (lseq) are identical to the rules for $L_{L R}$ (in Fig. 2), but the labeling algorithm is different.

Unlike $L_{L R}$, the normal order reduction of $L_{\text {name }}$ allows substitution of arbitrary expressions in (beta), (case), and (gcp) rules. An additional simplification (compared to $L_{L R}$ ) is that nested letrec-expressions are not flattened by reduction (i.e. there is no (llet)-reduction in $L_{\text {name }}$ ). As in $L_{L R}$ the normal order reduction of $L_{\text {name }}$ has reduction 
rules (lapp), (lcase), and (lseq) to move letrec-expressions out of an application, a seqexpression, or a case-expression.

Note that $\stackrel{\text { name }}{\longrightarrow}$ is unique. An $L_{n a m e}-\mathrm{WHNF}$ is defined as an expression either of the form $L[\lambda x . s]$ or of the form $L\left[\left(\begin{array}{cccc}c & \left.s_{1} \ldots s_{\text {ar }(c)}\right)\end{array}\right]\right.$ where $L$ is an $\mathcal{L}$ context. Let $\mathbb{A}_{\text {name }}$ be the set of $L_{\text {name }}-$ WHNFs, then the calculus $L_{\text {name }}$ is defined by the tuple $\left(\mathbb{E}_{\mathcal{L}}, \mathbb{C}_{\mathcal{L}}, \stackrel{\text { name }}{\longrightarrow}, \mathbb{A}_{\text {name }}\right)$ (modulo $\alpha$-equivalence).

3.3. The Extended Lazy Lambda Calculus $L_{l c c}$. In this subsection we give a short description of the lazy lambda calculus Abr90 extended by data constructors, case-expressions and seq-expressions, denoted with $L_{l c c}$. Unlike the calculi $L_{n a m e}$ and $L_{L R}$, this calculus has no letrec-expressions. The set $\mathbb{E}_{\lambda}$ of $L_{l c c}$-expressions is that of the usual (untyped) lambda calculus extended by data constructors, case, and seq:

$$
r, s, t, r_{i}, s_{i}, t_{i} \in \mathbb{E}_{\lambda}::=x|(s t)|(\lambda x . s)\left|\left(c s_{1} \ldots s_{\operatorname{ar}(c)}\right)\right|\left(\text { case }_{T} s \text { of alts }\right) \mid(\text { seq } s t)
$$

Contexts $\mathbb{C}_{\lambda}$ are $\mathbb{E}_{\lambda}$-expressions where a subexpression is replaced by the hole [·]. The set $\mathbb{A}_{l c c}$ of answers (or also values) are the $L_{l c c}$-abstractions and constructor applications. Reduction contexts $\mathcal{R}_{l c c}$ are defined by the following grammar, where $s$ is any $\mathbb{E}_{\lambda}$-expression:

$$
R_{l c c} \in \mathcal{R}_{l c c}:=[\cdot]\left|\left(R_{l c c} s\right)\right| \operatorname{case}_{T} R_{l c c} \text { of alts | seq } R_{l c c} s
$$

An $\stackrel{l c c}{\longrightarrow}$-reduction is defined by the three rules shown in Fig. 7, and thus the calculus $L_{l c c}$ is defined by the tuple $\left(\mathbb{E}_{\lambda}, \mathbb{C}_{\lambda}, \stackrel{l c c}{\longrightarrow}, \mathbb{A}_{l c c}\right)$ (modulo $\alpha$-equivalence).

$$
\begin{aligned}
& \text { (nbeta) } R_{l c c}[((\lambda x . s) t)] \stackrel{l c c}{\longrightarrow} R_{l c c}[s[t / x]] \\
& \text { (ncase) } R_{l c c}\left[\left(\operatorname{case}_{T}\left(c s_{1} \ldots s_{\operatorname{ar}(c)}\right) \text { of } \ldots\left(\left(c x_{1} \ldots x_{\operatorname{ar}(c)}\right) \rightarrow t\right) \ldots\right)\right] \\
& \stackrel{l c c}{\longrightarrow} t\left[s_{1} / x_{1}, \ldots, s_{\operatorname{ar}(c)} / x_{\operatorname{ar}(c)}\right] \\
& \text { (nseq) } \quad R_{l c c}[\text { seq } v s] \stackrel{l c c}{\longrightarrow} R_{l c c}[s] \text {, if } v \text { is an abstraction or a constructor application }
\end{aligned}
$$

Figure 7: Normal order reduction $\stackrel{l c c}{\longrightarrow}$ of $L_{l c c}$

\section{Properties of Similarity and Equivalences in $L_{l c c}$}

An applicative bisimilarity for $L_{l c c}$ and other alternative definitions are presented in subsection 4.2. As a preparation, we first analyze similarity for deterministic calculi in general.

4.1. Characterizations of Similarity in Deterministic Calculi. In this section we prove that for deterministic calculi (see Def. 2.1), the applicative similarity and its generalization to extended calculi, defined as the greatest fixpoint of an operator on relations, is equivalent to the inductive definition using Kleene's fixpoint theorem.

This implies that for deterministic calculi employing only beta-reduction, applicative similarity can be equivalently defined as $s \preccurlyeq t$, iff for all $n \geq 0$ and closed expressions $r_{i}, i=$ $1, \ldots, n$, the implication $\left(s r_{1} \ldots r_{n}\right) \downarrow_{D} \Longrightarrow\left(t r_{1} \ldots r_{n}\right) \downarrow_{D}$ holds, provided the calculus is convergence-admissible, which means that for all $r:(s r) \downarrow_{D} v \Longleftrightarrow \exists v^{\prime}: s \downarrow_{D} v^{\prime} \wedge\left(v^{\prime} r\right) \downarrow_{D} v$ (see Def. 4.5). 
This approach has a straightforward extension to calculi with other types of reductions, such as case- and seq-reductions. The calculi may also consist of a set of open expressions, contexts, and answers, as well as a subcalculus consisting of closed expressions, closed contexts and closed answers. We will use convergence-admissibility only for closed variants of the calculi.

In the following we assume $D=(\mathbb{E}, \mathbb{C}, \rightarrow, \mathbb{A})$ to be an untyped deterministic calculus and $\mathcal{Q} \subseteq \mathbb{C}$ be a set of functions on expressions. Note that the relations $\preccurlyeq_{D, \mathcal{Q}}$ and $\leq_{D, \mathcal{Q}}$ are defined in Definitions 2.5 and 2.6, respectively.

Lemma 4.1. For all expressions $s_{1}, s_{2} \in \mathbb{E}$ the following holds: $s_{1} \preccurlyeq D, \mathcal{Q} s_{2}$ if, and only if, $s_{1} \downarrow_{D} v_{1} \Longrightarrow\left(s_{2} \downarrow_{D} v_{2} \wedge \forall Q \in \mathcal{Q}: Q\left(v_{1}\right) \preccurlyeq{ }_{D, \mathcal{Q}} Q\left(v_{2}\right)\right)$.

Proof. Since $\preccurlyeq D, \mathcal{Q}$ is a fixpoint of $F_{D, \mathcal{Q}}$, we have $\preccurlyeq_{D, \mathcal{Q}}=F_{D, \mathcal{Q}}\left(\preccurlyeq_{D, \mathcal{Q}}\right)$. This equation is equivalent to the claim of the lemma.

Now we show that the operator $F_{D, \mathcal{Q}}$ is lower-continuous, and thus we can apply Kleene's fixpoint theorem to derive an alternative characterization of $\preccurlyeq_{D, \mathcal{Q}}$.

For infinite chains of sets $S_{1}, S_{2} \ldots$, we define the greatest lower bound w.r.t. setinclusion ordering as $\operatorname{glb}\left(S_{1}, S_{2}, \ldots\right)=\bigcap_{i=1}^{\infty} S_{i}$.

Proposition 4.2. $F_{\mathcal{Q}}$ is lower-continuous w.r.t. countably infinite descending chains $C=$ $\eta_{1} \supseteq \eta_{2} \supseteq \ldots$, i.e. $\operatorname{glb}\left(F_{\mathcal{Q}}(C)\right)=F_{\mathcal{Q}}(\operatorname{glb}(C))$ where $F_{\mathcal{Q}}(C)$ is the infinite descending chain $F_{\mathcal{Q}}\left(\eta_{1}\right) \supseteq F_{\mathcal{Q}}\left(\eta_{2}\right) \supseteq \ldots$

Proof. " $\supseteq$ ": Since $\operatorname{glb}(C)=\bigcap_{i=1}^{\infty} \eta_{i}$, we have for all $i: \operatorname{glb}(C) \subseteq \eta_{i}$. Applying monotonicity of $F_{\mathcal{Q}}$ yields $F_{\mathcal{Q}}(\operatorname{glb}(C)) \subseteq F_{\mathcal{Q}}\left(\eta_{i}\right)$ for all $i$. This implies $F_{\mathcal{Q}}(\operatorname{glb}(C)) \subseteq \bigcap_{i=1}^{\infty} F_{\mathcal{Q}}\left(\eta_{i}\right)$, i.e. $F_{\mathcal{Q}}(\operatorname{glb}(C)) \subseteq \operatorname{glb}\left(F_{\mathcal{Q}}(C)\right)$

"ᄃ": Let $\left(s_{1}, s_{2}\right) \in \operatorname{glb}\left(F_{\mathcal{Q}}(C)\right)$, i.e. for all $i:\left(s_{1}, s_{2}\right) \in F_{\mathcal{Q}}\left(\eta_{i}\right)$. Unfolding the definition of $F_{\mathcal{Q}}$ gives: $\forall i: s_{1} \downarrow_{D} v_{1} \Longrightarrow\left(s_{2} \downarrow_{D} v_{2} \wedge \forall Q \in \mathcal{Q}: Q\left(v_{1}\right) \eta_{i} Q\left(v_{2}\right)\right)$. Now we can move the universal quantifier for $i$ inside the formula: $s_{1} \downarrow_{D} v_{1} \Longrightarrow\left(s_{2} \downarrow_{D} v_{2} \wedge \forall Q \in \mathcal{Q}: \forall \mathrm{i}\right.$ : $\left.Q\left(v_{1}\right) \eta_{i} Q\left(v_{2}\right)\right)$. This is equivalent to $s_{1} \downarrow_{D} v_{1} \Longrightarrow\left(s_{2} \downarrow_{D} v_{2} \wedge \forall Q \in \mathcal{Q}: Q\left(v_{1}\right)\left(\bigcap_{i=1}^{\infty} \eta_{i}\right) Q\left(v_{2}\right)\right)$ or $s_{1} \downarrow_{D} v_{1} \Longrightarrow\left(s_{2} \downarrow_{D} v_{2} \wedge \forall Q \in \mathcal{Q}:\left(Q\left(v_{1}\right), Q\left(v_{2}\right)\right) \in \operatorname{glb}(C)\right)$ and thus $\left(s_{1}, s_{2}\right) \in F_{\mathcal{Q}}(\operatorname{glb}(C))$.

Definition 4.3. Let $\preccurlyeq D, \mathcal{Q}, i$ for $i \in \mathbb{N}_{0}$ be defined as follows:

$$
\preccurlyeq_{D, \mathcal{Q}, 0}=\mathbb{E} \times \mathbb{E} \text { and } \preccurlyeq_{D, \mathcal{Q}, i}=F_{D, \mathcal{Q}}\left(\preccurlyeq_{D, \mathcal{Q}, i-1}\right) \text { if } i>0
$$

Theorem 4.4. $\preccurlyeq D, \mathcal{Q}=\bigcap_{i=1}^{\infty} \preccurlyeq D, \mathcal{Q}, i$

Proof. The claim follows from Kleene's fixpoint theorem, since $F_{\mathcal{Q}}$ is monotonous and lowercontinuous, and since $\preccurlyeq D, \mathcal{Q}, i+1 \subseteq \preccurlyeq D, \mathcal{Q}, i$ for all $i \geq 0$.

This representation of $\preccurlyeq D, \mathcal{Q}$ allows inductive proofs to show similarity. Now we show that $\mathcal{Q}$-similarity is identical to $\leq_{D, \mathcal{Q}}$ under moderate conditions, i.e. our characterization result will only apply if the underlying calculus is convergence-admissible w.r.t. $\mathcal{Q}$ :

Definition 4.5. An untyped deterministic calculus $(\mathbb{E}, \mathbb{C}, \rightarrow, \mathbb{A})$ is convergence-admissible w.r.t. $\mathcal{Q}$ if, and only if $\forall Q \in \mathcal{Q}, s \in \mathbb{E}, v \in \mathbb{A}: Q(s) \downarrow_{D} v \Longleftrightarrow \exists v^{\prime}: s \downarrow_{D} v^{\prime} \wedge Q\left(v^{\prime}\right) \downarrow_{D} v$ 
Convergence-admissibility can be seen as a restriction on choosing the set $\mathcal{Q}$ : In most calculi (subsets of) reduction contexts satisfy the property for convergence-admissibility, while including non-reduction contexts into $\mathcal{Q}$ usually breaks convergence-admissibility.

Lemma 4.6. Let $(\mathbb{E}, \mathbb{C}, \rightarrow, \mathbb{A})$ be convergence-admissible w.r.t. $\mathcal{Q}$. Then the following holds:

- $s_{1} \leq_{D, \mathcal{Q}} s_{2} \Longrightarrow Q\left(s_{1}\right) \leq_{D, \mathcal{Q}} Q\left(s_{2}\right)$ for all $Q \in \mathcal{Q}$

- $s_{1} \leq_{D, \mathcal{Q}} s_{2}, s_{1} \downarrow_{D} v_{1}$, and $s_{2} \downarrow_{D} v_{2} \Longrightarrow v_{1} \leq_{D, \mathcal{Q}} v_{2}$

Proof. The first part is easy to verify. For the second part it is important that $D$ is deterministic. Let $s_{1} \leq_{D, \mathcal{Q}} s_{2}$, and $s_{1} \downarrow_{D} v_{1}, s_{2} \downarrow_{D} v_{2}$ hold. Assume that $Q_{1}\left(\ldots\left(Q_{n}\left(v_{1}\right)\right)\right) \downarrow_{D} v_{1}^{\prime}$ for some $n \geq 0$ where all $Q_{i} \in \mathcal{Q}$. Convergence-admissibility implies $Q_{1}\left(\ldots\left(\left(Q_{n}\left(s_{1}\right)\right)\right)\right) \downarrow_{D} v_{1}^{\prime}$. Now $s_{1} \leq{ }_{D, \mathcal{Q}} s_{2}$ implies $Q_{1}\left(\ldots\left(Q_{n}\left(s_{2}\right)\right)\right) \downarrow_{D} v_{2}^{\prime}$. Finally, convergence-admissibility (applied multiple times) shows that $s_{2} \downarrow_{D} v_{2}$ and $Q_{1}\left(\ldots\left(Q_{n}\left(v_{2}\right)\right)\right) \downarrow_{D} v_{2}^{\prime}$ holds.

We prove that $\preccurlyeq D, \mathcal{Q}$ respects functions $Q \in \mathcal{Q}$ provided the underlying deterministic calculus is convergence-admissible w.r.t. $\mathcal{Q}$ :

Lemma 4.7. Let $(\mathbb{E}, \mathbb{C}, \rightarrow, \mathbb{A})$ be convergence-admissible w.r.t. $\mathcal{Q}$. Then for all $s_{1}, s_{2} \in E:$ $s_{1} \preccurlyeq D, \mathcal{Q} s_{2} \Longrightarrow Q\left(s_{1}\right) \preccurlyeq D, \mathcal{Q} Q\left(s_{2}\right)$ for all $Q \in \mathcal{Q}$

Proof. Let $s_{1} \preccurlyeq_{D, \mathcal{Q}} s_{2}, Q_{0} \in \mathcal{Q}$, and $Q_{0}\left(s_{1}\right) \downarrow_{D} v_{1}$. By convergence admissibility $s_{1} \downarrow_{D} v_{1}^{\prime}$ holds and $Q_{0}\left(v_{1}^{\prime}\right) \downarrow_{D} v_{1}$. Since $s_{1} \preccurlyeq{ }_{D, \mathcal{Q}} s_{2}$ this implies $s_{2} \downarrow_{D} v_{2}^{\prime}$ and for all $Q \in \mathcal{Q}: Q\left(v_{1}^{\prime}\right) \preccurlyeq D, \mathcal{Q} Q\left(v_{2}^{\prime}\right)$. Hence, from $Q_{0}\left(v_{1}^{\prime}\right) \downarrow_{D} v_{1}$ we derive $Q_{0}\left(v_{2}^{\prime}\right) \downarrow_{D} v_{2}$. Convergence admissibility now implies $Q_{0}\left(s_{2}\right) \downarrow_{D} v_{2}$.

It remains to show for all $Q \in \mathcal{Q}: Q\left(v_{1}\right) \preccurlyeq{ }_{D, \mathcal{Q}} Q\left(v_{2}\right)$ : Since $Q_{0}\left(v_{1}^{\prime}\right) \downarrow_{D} v_{1}$ and $Q_{0}\left(v_{2}^{\prime}\right) \downarrow_{D} v_{2}$, applying Lemma 4.1 to $Q_{0}\left(v_{1}^{\prime}\right) \preccurlyeq_{D, \mathcal{Q}} Q_{0}\left(v_{2}^{\prime}\right)$ implies $Q\left(v_{1}\right) \preccurlyeq_{D, \mathcal{Q}} Q\left(v_{2}\right)$ for all $Q \in \mathcal{Q}$.

We now prove that $\leq_{D, \mathcal{Q}}$ and $\mathcal{Q}$-similarity coincide for convergence-admissible deterministic calculi:

Theorem 4.8. Let $(\mathbb{E}, \mathbb{C}, \rightarrow, \mathbb{A})$ be convergence-admissible w.r.t. $\mathcal{Q}$. Then $\leq_{D, \mathcal{Q}}=\preccurlyeq D, \mathcal{Q}$.

Proof. "ᄃ": Let $s_{1} \leq_{D, \mathcal{Q}} s_{2}$. We use Theorem 4.4 and show $s_{1} \preccurlyeq D, \mathcal{Q}, i s_{2}$ for all $i$. We use induction on $i$. The base case $(i=0)$ obviously holds. Let $i>0$ and let $s_{1} \downarrow_{D} v_{1}$. Then $s_{1} \leq_{D, \mathcal{Q}} s_{2}$ implies $s_{2} \downarrow_{D} v_{2}$. Thus, it is sufficient to show that $Q\left(v_{1}\right) \preccurlyeq_{D, \mathcal{Q}, i-1} Q\left(v_{2}\right)$ for all $Q \in \mathcal{Q}$ : As induction hypothesis we use that $s_{1} \leq_{D, \mathcal{Q}} s_{2} \Longrightarrow s_{1} \preccurlyeq_{D, \mathcal{Q}, i-1} s_{2}$ holds. Using Lemma 4.6 twice and $s_{1} \leq_{D, \mathcal{Q}} s_{2}$, we have $Q\left(v_{1}\right) \leq_{D, \mathcal{Q}} Q\left(v_{2}\right)$. The induction hypothesis shows that $Q\left(v_{1}\right) \preccurlyeq D, \mathcal{Q}, i-1 \quad Q\left(v_{2}\right)$. Now the definition of $\preccurlyeq D, \mathcal{Q}, i$ is satisfied, which shows $s_{1} \preccurlyeq D, \mathcal{Q}, i s_{2}$.

"卫": Let $s_{1} \preccurlyeq D, \mathcal{Q} s_{2}$. By induction on the number $n$ of $Q$-contexts we show $\forall n, Q_{i} \in \mathcal{Q}$ : $Q_{1}\left(\ldots\left(Q_{n}\left(s_{1}\right)\right)\right) \downarrow_{D} \Longrightarrow Q_{1}\left(\ldots\left(Q_{n}\left(s_{2}\right)\right)\right) \downarrow_{D}$. The base case follows from $s_{1} \preccurlyeq D, \mathcal{Q} s_{2}$. For the induction step we use the following induction hypothesis: $t_{1} \preccurlyeq D, \mathcal{Q} t_{2} \Longrightarrow \forall j<n, Q_{i} \in$ $\mathcal{Q}: Q_{1}\left(\ldots\left(Q_{j}\left(t_{1}\right)\right)\right) \downarrow_{D} \Longrightarrow Q_{1}\left(\ldots\left(Q_{j}\left(t_{2}\right)\right)\right) \downarrow_{D}$ for all $t_{1}, t_{2}$. Let $Q_{1}\left(\ldots\left(Q_{n}\left(s_{1}\right)\right)\right) \downarrow_{D}$. From Lemma 4.7 we have $r_{1} \preccurlyeq D, \mathcal{Q} r_{2}$, where $r_{i}=Q_{n}\left(s_{i}\right)$. Now the induction hypothesis shows that $Q_{1}\left(\ldots\left(Q_{n-1}\left(r_{1}\right)\right)\right) \downarrow_{D} \Longrightarrow Q_{1}\left(\ldots\left(Q_{n-1}\left(r_{2}\right)\right)\right) \downarrow_{D}$ and thus $Q_{1}\left(\ldots\left(Q_{n}\left(s_{2}\right)\right)\right) \downarrow_{D}$. 


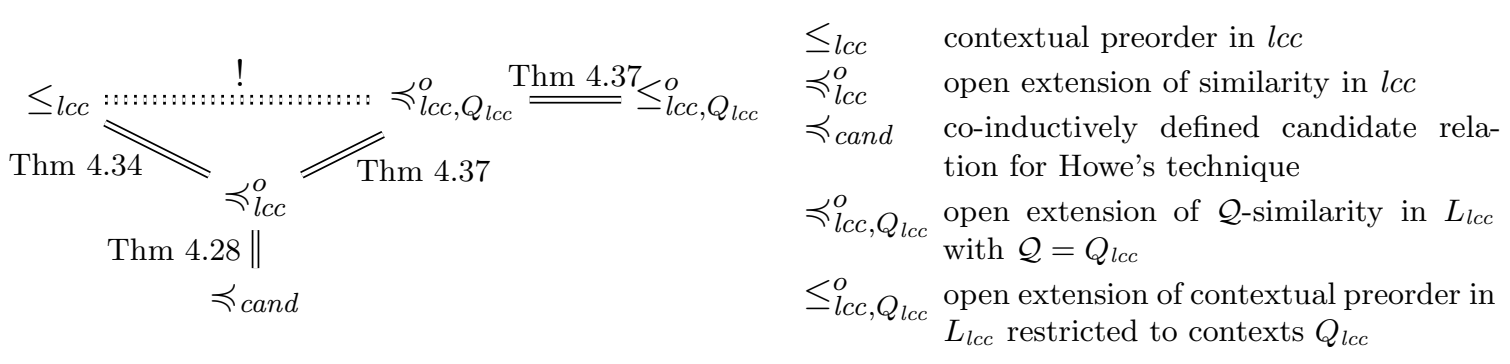

Figure 8: Structure of soundness and completeness proofs for similarities in $L_{l c c}$. The $=!=$ indicates a required equality which can only be proved via Howe's technique.

4.2. Applicative Simulation in $L_{l c c}$. In this section we will show that applicative similarity and contextual preorder coincide in $L_{l c c}$.

Notation. In abuse of notation we use higher order abstract syntax as e.g. in [How89] for the proof and write $\tau(.$.$) for an expression with top operator \tau$, which may be all possible term constructors, like case, application, a constructor, seq, or $\lambda$, and $\theta$ for an operator that may be the head of a value, i.e. a constructor or $\lambda$.

Definition 4.9. For a relation $\eta$ on closed $\mathbb{E}_{\lambda}$-expressions $\eta^{o}$ is the open extension on $L_{l c c}$ :

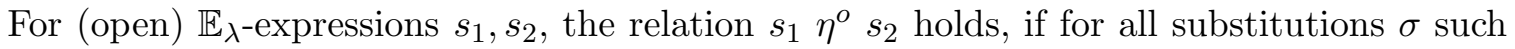
that $\sigma\left(s_{1}\right), \sigma\left(s_{2}\right)$ are closed, the relation $\sigma\left(s_{1}\right) \eta \sigma\left(s_{2}\right)$ holds. Conversely, for binary relations $\mu$ on open expressions, $(\mu)^{c}$ is the restriction to closed expressions.

We say a binary relation $\mu$ is operator-respecting, iff $s_{i} \mu t_{i}$ for $i=1, \ldots, n$ implies $\tau\left(s_{1}, \ldots, s_{n}\right) \mu \tau\left(t_{1}, \ldots, t_{n}\right)$.

Note that $\tau$ and $\theta$ may also represent the binding $\lambda$ using $\lambda(x . s)$ as representing $\lambda$ x.s. For consistency of terminology and treatment with that in other papers such as [How89, we assume that removing the top constructor $\lambda x$ in relations is done after a renaming. For example, $\lambda x . s \mu \lambda y . t$ is renamed before further treatment to $\lambda z . s[z / x] \mu \lambda z . t[z / y]$ for a fresh variable $z$.

Plan of Subsection 4.2. We start by explaining the subgoals of the soundness and completeness proofs for similarities in $L_{l c c}$ and its structure, illustrated in Fig. 8, The main result we want to show is that contextual preorder $\leq_{l c c}$ and $\preccurlyeq_{l c c, Q_{l c c}}^{o}$ coincide, where $\preccurlyeq_{l c c, Q_{l c c}}^{o}$ is the open extension of $\preccurlyeq_{l c c, Q_{l c c}}$, and $\preccurlyeq_{l c c, Q_{l c c}}$ is $\mathcal{Q}$-similarity introduced in Definition 2.5 instantiated with the subcalculus of $L_{l c c}$ which consists of closed expressions, closed contexts, and closed answers, and $Q_{l c c}$ is a specific set of small closed $L_{l c c}$-contexts. $\mathcal{Q}$-similarity does not allow a direct proof of soundness and completeness for contextual equivalence using Howe's method [How89, How96], since it is not stated in terms of the syntactic form of values derived by evaluation. We overcome this obstacle by defining another similarity $\preccurlyeq l c c$ in $L_{l c c}$ for which we will perform the proof of soundness and completeness w.r.t. contextual preorder. Since the definition of $\preccurlyeq_{l c c}$ does not obviously imply that $\preccurlyeq_{l c c}$ is a precongruence, a candidate relation $\preccurlyeq$ cand is defined, which is trivially compatible with contexts, but needs to be shown to be transitive. After proving $\preccurlyeq_{c a n d}=\preccurlyeq_{l c c}^{o}$, i.e. that $\preccurlyeq_{l c c}^{o}$ is a precongruence, soundness of $\preccurlyeq_{l c c}^{o}$ w.r.t. contextual preorder $\leq_{l c c}$ follows. Completeness can then also be proven. In a second step we prove that $\preccurlyeq_{l c c, Q_{l c c}}^{o}$ is sound and complete for contextual equivalence, i.e. $\leq_{l c c}=\preccurlyeq_{l c c, Q_{l c c}}^{o}$. After showing that $L_{l c c}$ is convergence-admissible we are 
also able to show that the inductive description $\leq_{l c c, Q_{l c c}}$ of $\mathcal{Q}$-similarity coincides with $\preccurlyeq_{l c c, Q_{l c c}}$.

Another obstacle is that the contextual preorder contains the irregularity $\lambda x . \Omega \leq_{l c c}$ $c s_{1} \ldots s_{n}$ for any constructor $c$. This requires an adapted definition of the similarity relation, and a slightly modified proof route.

In the following let $c B$ ot be the set of $\mathbb{E}_{\lambda}$-expressions $s$ with the property that for all

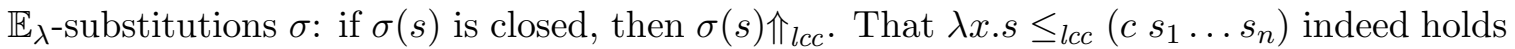
is shown in Proposition 4.32, Now we define an applicative similarity $\preccurlyeq_{l c c}$ in $L_{l c c}$ analogous to [How89, How96], where this irregularity is taken into account.

Definition 4.10 (Similarity in $L_{l c c}$ ). Let $\eta$ be a binary relation on closed $\mathbb{E}_{\lambda}$-expressions. Let $F_{l c c}$ be the following operator on relations on closed $\mathbb{E}_{\lambda}$-expressions:

$s F_{l c c}(\eta) t$ holds iff

- $s \downarrow_{l c c} \lambda x . s^{\prime} \Longrightarrow\left(t \downarrow_{l c c} \lambda x . t^{\prime}\right.$ and $s^{\prime} \eta^{o} t^{\prime}$, or $t \downarrow_{l c c}\left(c t_{1}^{\prime} \ldots t_{n}^{\prime}\right)$ and $\left.s^{\prime} \in c B o t\right)$

- $s \downarrow_{l c c}\left(c s_{1}^{\prime} \ldots s_{n}^{\prime}\right) \Longrightarrow\left(t \downarrow_{l c c}\left(c t_{1}^{\prime} \ldots t_{n}^{\prime}\right)\right.$ and the relation $s_{i}^{\prime} \eta t_{i}^{\prime}$ holds for all $\left.i\right)$

Similarity $\preccurlyeq_{l c c}$ is defined as the greatest fixpoint of the operator $F_{l c c}$. Bisimilarity $\simeq_{l c c}$ is defined as $s \simeq_{l c c} t$ iff $s \preccurlyeq l c c t \wedge t \preccurlyeq l c c s$.

Note that the operator $F_{l c c}$ is monotone, hence the greatest fixpoint $\preccurlyeq l c c$ exists.

4.2.1. Similarity and Contextual Preorder Coincide in $L_{l c c}$. Although applying Howe's proof technique is standard, for the sake of completeness, and to demonstrate that the irregularity $\lambda x . \Omega \leq_{l c c}\left(c s_{1} \ldots s_{n}\right)$ can also be treated, we will explicitly show in this section that $\preccurlyeq_{l c c}^{o}=\leq_{l c c}$ using Howe's method [How89, How96].

Lemma 4.11. For a relation $\eta$ on closed expressions it holds $\left((\eta)^{o}\right)^{c}=\eta$, and also $s \eta^{o} t$ implies $\sigma(s) \eta^{o} \sigma(t)$ for any substitution $\sigma$. For a relation $\mu$ on open expressions, $\mu \subseteq$ $\left((\mu)^{c}\right)^{o}$ is equivalent to $s \mu t \Longrightarrow \sigma(s)(\mu)^{c} \sigma(t)$ for all closing substitutions $\sigma$.

Proposition 4.12 (Co-Induction). The principle of co-induction for the greatest fixpoint of $F_{l c c}$ shows that for every relation $\eta$ on closed expressions with $\eta \subseteq F_{l c c}(\eta)$, we derive $\eta \subseteq \preccurlyeq_{l c c}$. This obviously also implies $(\eta)^{\circ} \subseteq\left(\preccurlyeq_{l c c}\right)^{o}$.

The fixpoint property of $\preccurlyeq_{l c c}$ implies:

Lemma 4.13. For a closed value $\theta_{1}\left(s_{1}, \ldots, s_{n}\right)$, and a closed term $t$ with $\theta_{1}\left(s_{1}, \ldots, s_{n}\right) \preccurlyeq l c c$ $t$, we have $t_{\downarrow_{l c c}} \theta_{2}\left(t_{1}, \ldots, t_{n}\right)$, and there are two cases:

(1) $\theta_{1}=\theta_{2}$ are constructors or $\lambda$ and $s_{i} \preccurlyeq_{l c c}^{o} t_{i}$ for all $i$.

(2) $\theta_{1}\left(s_{1}, \ldots, s_{n}\right)=\lambda(x . s)$ with $s \in c B$ ot and $\theta_{2}$ is a constructor.

Lemma 4.14. For two expressions $s, t: s \in c$ Bot implies $s \preccurlyeq_{l c c}^{o} t$. Thus any two expressions $s, t \in c$ Bot are bisimilar: $s \simeq_{l c c}^{o} t$.

Particular expressions in $c B o t$ are (case $(\lambda x . s)$ alts $)$ and $\left(c\left(s_{1}, \ldots, s_{n}\right) a_{1} \ldots a_{m}\right)$ for $m \geq 1$; also $s \in c B o t$ implies that $(s t)$, (seq $s t$ ), (case $s$ alts) and $\sigma(s)$ are also in $c B o t$.

Lemma 4.15. The relations $\preccurlyeq_{l c c}$ and $\preccurlyeq_{l c c}^{o}$ are reflexive and transitive.

Proof. Reflexivity follows by showing that $\eta:=\preccurlyeq l c c \cup\left\{(s, s) \mid s \in \mathbb{E}_{\lambda}, s\right.$ closed $\}$ satisfies $\eta \subseteq F_{l c c}(\eta)$. Transitivity follows by showing that $\eta:=\preccurlyeq_{l c c} \cup\left(\preccurlyeq_{l c c} \circ \preccurlyeq_{l c c}\right)$ satisfies $\eta \subseteq F_{l c c}(\eta)$ and then using the co-induction principle. 
The goal in the following is to show that $\preccurlyeq_{l c c}$ is a precongruence. This proof proceeds by defining a precongruence candidate $\preccurlyeq_{\text {cand }}$ as a closure of $\preccurlyeq_{l c c}$ within contexts, which obviously is operator-respecting, but transitivity needs to be shown. By proving that $\preccurlyeq_{l c c}^{o}$ and $\preccurlyeq$ cand coincide, on the one hand transitivity of $\preccurlyeq_{\text {cand }}$ follows (since $\preccurlyeq_{l c c}$ is transitive) and on the other hand (and more importantly) it follows that $\preccurlyeq_{l c c}^{o}$ is operator-respecting (since $\preccurlyeq_{\text {cand }}$ is operator-respecting) and thus a precongruence.

Definition 4.16. The precongruence candidate $\preccurlyeq$ cand is a binary relation on open expressions and is defined as the greatest fixpoint of the monotone operator $F_{\text {cand }}$ on relations on all expressions:

(1) $x F_{\text {cand }}(\eta) s$ iff $x \preccurlyeq_{l c c}^{o} s$.

(2) $\tau\left(s_{1}, \ldots, s_{n}\right) F_{\text {cand }}(\eta) s$ iff there is some expression $\tau\left(s_{1}^{\prime}, \ldots, s_{n}^{\prime}\right) \preccurlyeq_{l c c}^{o} s$ with $s_{i} \eta s_{i}^{\prime}$ for $i=1, \ldots, n$.

Lemma 4.17. If some relation $\eta$ satisfies $\eta \subseteq F_{\text {cand }}(\eta)$, then $\eta \subseteq \preccurlyeq$ cand .

Since $\preccurlyeq$ cand is a fixpoint of $F_{\text {cand }}$, we have:

\section{Lemma 4.18.}

(1) $x \preccurlyeq_{\text {cand }} s$ iff $x \preccurlyeq_{l c c}^{o} s$.

(2) $\tau\left(s_{1}, \ldots, s_{n}\right) \preccurlyeq$ cand $s$ iff there is some expression $\tau\left(s_{1}^{\prime}, \ldots, s_{n}^{\prime}\right) \preccurlyeq_{l c c}^{o} s$ with $s_{i} \preccurlyeq_{\text {cand }} s_{i}^{\prime}$ for $i=1, \ldots, n$.

Some technical facts about the precongruence candidate are now proved:

Lemma 4.19. The following properties hold:

$(1) \preccurlyeq$ cand is reflexive.

$(2) \preccurlyeq$ cand and $\left(\preccurlyeq_{\text {cand }}\right)^{c}$ are operator-respecting.

$(3) \preccurlyeq_{l c c}^{o} \subseteq \preccurlyeq_{\text {cand }}$ and $\preccurlyeq_{l c c} \subseteq\left(\preccurlyeq_{\text {cand }}\right)^{c}$.

(4) $\preccurlyeq_{\text {cand }} \circ \preccurlyeq_{\text {lcc }}^{o} \subseteq \preccurlyeq$ cand .

(5) $\left(s \preccurlyeq_{\text {cand }} s^{\prime} \wedge t \preccurlyeq_{\text {cand }} t^{\prime}\right) \Longrightarrow t[s / x] \preccurlyeq_{\text {cand }} t^{\prime}\left[s^{\prime} / x\right]$.

(6) $s \preccurlyeq$ cand $t$ implies that $\sigma(s) \preccurlyeq$ cand $\sigma(t)$ for every substitution $\sigma$.

$(7) \preccurlyeq$ cand $\subseteq\left(\left(\preccurlyeq_{\text {cand }}\right)^{c}\right)^{o}$

Proof. Parts (11) - (3) can be shown by structural induction and using reflexivity of $\preccurlyeq_{l c c}^{o}$. Part (4) follows from the definition, Lemma 4.18, and transitivity of $\preccurlyeq_{l c c}^{o}$.

For part (5) let $\eta:=\preccurlyeq$ cand $\cup\left\{\left(r[s / x], r^{\prime}\left[s^{\prime} / x\right]\right) \mid r \preccurlyeq\right.$ cand $\left.r^{\prime}\right\}$. Using co-induction it suffices to show that $\eta \subseteq F_{\text {cand }}(\eta)$ : In the case $x \preccurlyeq$ cand $r^{\prime}$, we obtain $x \preccurlyeq_{l c c}^{o} r^{\prime}$ from the definition, and $s^{\prime} \preccurlyeq_{l c c}^{o} r^{\prime}\left[s^{\prime} / x\right]$ and thus $x[s / x] \preccurlyeq$ cand $r^{\prime}\left[s^{\prime} / x\right]$. In the case $y \preccurlyeq$ cand $r$, we obtain $y \preccurlyeq_{l c c}^{o} r^{\prime}$ from the definition, and $y[s / x]=y \preccurlyeq_{l c c}^{o} r^{\prime}\left[s^{\prime} / x\right]$ and thus $y=y[s / x] \preccurlyeq c a n d r^{\prime}\left[s^{\prime} / x\right]$. If $r=\tau\left(r_{1}, \ldots, r_{n}\right), r \preccurlyeq$ cand $r^{\prime}$ and $r[s / x] \eta r^{\prime}\left[s^{\prime} / x\right]$, then there is some $\tau\left(r_{1}^{\prime}, \ldots, r_{n}^{\prime}\right) \preccurlyeq_{l c c}^{o} r^{\prime}$ with $r_{i} \preccurlyeq$ cand $r_{i}^{\prime}$. W.l.o.g. bound variables have fresh names. We have $r_{i}[s / x] \eta r_{i}^{\prime}\left[s^{\prime} / x\right]$ and $\tau\left(r_{1}^{\prime}, \ldots, r_{n}^{\prime}\right)\left[s^{\prime} / x\right] \preccurlyeq_{l c c}^{o} r^{\prime}\left[s^{\prime} / x\right]$. Thus $r[s / x] F_{\text {cand }}(\eta) r^{\prime}\left[s^{\prime} / x\right]$.

Part (66) follows from item (5). Part (7) follows from item ([6) and Lemma 4.11]

Lemma 4.20. The middle expression in the definition of $\preccurlyeq_{\text {cand }}$ can be chosen to be closed if $s, t$ are closed: Let $s=\tau\left(s_{1}, \ldots, s_{\operatorname{ar}(\tau)}\right)$, such that $s \preccurlyeq$ cand $t$ holds. Then there are operands $s_{i}^{\prime}$, such that $\tau\left(s_{1}^{\prime}, \ldots, s_{\operatorname{ar}(\tau)}^{\prime}\right)$ is closed, $\forall i: s_{i} \preccurlyeq$ cand $s_{i}^{\prime}$ and $\tau\left(s_{1}^{\prime}, \ldots, s_{\operatorname{ar}(\tau)}^{\prime}\right) \preccurlyeq_{l c c}^{o} t$. 
Proof. The definition of $\preccurlyeq$ cand implies that there is an expression $\tau\left(s_{1}^{\prime \prime}, \ldots, s_{\operatorname{ar}(\tau)}^{\prime \prime}\right)$ such that $s_{i} \preccurlyeq$ cand $s_{i}^{\prime \prime}$ for all $i$ and $\tau\left(s_{1}^{\prime \prime}, \ldots, s_{\operatorname{ar}(\tau)}^{\prime \prime}\right) \preccurlyeq_{l c c}^{o} t$. Let $\sigma$ be the substitution with $\sigma(x):=r_{x}$ for all $x \in F V\left(\tau\left(s_{1}^{\prime \prime}, \ldots, s_{\operatorname{ar}(\tau)}^{\prime \prime}\right)\right)$, where $r_{x}$ is any closed expression. Lemma 4.19 now shows that $s_{i}=\sigma\left(s_{i}\right) \preccurlyeq_{\text {cand }} \sigma\left(s_{i}^{\prime \prime}\right)$ holds for all $i$. The relation $\sigma\left(\tau\left(s_{1}^{\prime \prime}, \ldots, s_{\operatorname{ar}(\tau)}^{\prime \prime}\right)\right) \preccurlyeq_{l c c}^{o} t$ holds, since $t$ is closed and due to the definition of an open extension. The requested expression is $\tau\left(\sigma\left(s_{1}^{\prime \prime}\right), \ldots, \sigma\left(s_{\operatorname{ar}(\tau)}^{\prime \prime}\right)\right)$.

Since reduction $\stackrel{l c c}{\longrightarrow}$ is deterministic:

Lemma 4.21. If $s \stackrel{l c c}{\longrightarrow} s^{\prime}$, then $s^{\prime} \preccurlyeq_{l c c}^{o} s$ and $s \preccurlyeq_{l c c}^{o} s^{\prime}$.

Lemmas 4.21 and 4.19 imply that $\preccurlyeq$ cand is right-stable w.r.t. reduction:

Lemma 4.22. If $s \preccurlyeq$ cand $t$ and $t \stackrel{l c c}{\longrightarrow} t^{\prime}$, then $s \preccurlyeq$ cand $t^{\prime}$.

We show that $\preccurlyeq$ cand is left-stable w.r.t. reduction:

Lemma 4.23. Let $s, t$ be closed expressions such that $s=\theta\left(s_{1}, \ldots, s_{n}\right)$ is a value and $s \preccurlyeq$ cand $t$. Then there are two possibilities:

(1) $s=\lambda x . s_{1}$ and $t \downarrow_{l c c} c\left(t_{1}, \ldots, t_{n}\right)$, where $c$ is a constructor;

(2) there is some closed value $t^{\prime}=\theta\left(t_{1}, \ldots, t_{n}\right)$ with $t \stackrel{l c c, *}{\longrightarrow} t^{\prime}$ and for all $i: s_{i} \preccurlyeq$ cand $t_{i}$.

Proof. The definition of $\preccurlyeq$ cand implies that there is a closed expression $\theta\left(t_{1}^{\prime}, \ldots, t_{n}^{\prime}\right)$ with $s_{i} \preccurlyeq_{c a n d} t_{i}^{\prime}$ for all $i$ and $\theta\left(t_{1}^{\prime}, \ldots, t_{n}^{\prime}\right) \preccurlyeq_{l c c} t$. Lemma 4.13 implies that $t_{l c c}$, hence either $t \stackrel{l c c, *}{\longrightarrow} c\left(t_{1}^{\prime \prime}, \ldots, t_{n}^{\prime \prime}\right)$ or $t \stackrel{l c c, *}{\longrightarrow} \lambda x \cdot t_{1}^{\prime \prime}$.

- First let $\theta=\lambda$. The case $t \stackrel{l c c, *}{\longrightarrow} c\left(t_{1}^{\prime \prime}, \ldots, t_{n}^{\prime \prime}\right)$ is possibility (1) of the lemma.

In the second case, $t \stackrel{l c c, *}{\longrightarrow} \lambda x \cdot t_{1}^{\prime \prime}$, Lemma 4.22 implies $\lambda x . s_{1} \preccurlyeq$ cand $\lambda x . t_{1}^{\prime \prime}$. Definition of $\preccurlyeq$ cand and Lemma 4.20 now show that there is some closed $\lambda x . t_{1}^{\prime \prime \prime}$ with $s_{1} \preccurlyeq$ cand $t_{1}^{\prime \prime \prime}$ and $\lambda x \cdot t_{1}^{\prime \prime \prime} \preccurlyeq_{l c c} \lambda x \cdot t_{1}^{\prime \prime}$. The latter relation implies $t_{1}^{\prime \prime \prime} \preccurlyeq_{l c c}^{o} t_{1}^{\prime \prime}$, which shows $s_{1}^{\prime} \preccurlyeq_{c a n d} t_{1}^{\prime \prime}$ by Lemma 4.19 (41).

- If $\theta$ is a constructor, then there is a closed expression $\theta\left(t_{1}^{\prime}, \ldots, t_{n}^{\prime}\right)$ with $s_{i} \preccurlyeq$ cand $t_{i}^{\prime}$ for all $i$ and $\theta\left(t_{1}^{\prime}, \ldots, t_{n}^{\prime}\right) \preccurlyeq l c c t$. The properties of $\preccurlyeq_{l c c}$ imply that $t \stackrel{l c c, *}{\longrightarrow} \theta\left(t_{1}^{\prime \prime}, \ldots, t_{n}^{\prime \prime}\right)$ with $t_{i}^{\prime} \preccurlyeq l c c t_{i}^{\prime \prime}$ for all $i$. By definition of $\preccurlyeq$ cand and Lemma 4.19 (4), we obtain $s_{i} \preccurlyeq$ cand $t_{i}^{\prime \prime}$ for all $i$.

Proposition 4.24. Let $s, t$ be closed expressions, $s \preccurlyeq$ cand $t$ and $s \stackrel{l c c}{\longrightarrow} s^{\prime}$ where $s$ is the redex. Then $s^{\prime} \preccurlyeq$ cand $t$.

Proof. The relation $s \preccurlyeq$ cand $t$ implies that $s=\tau\left(s_{1}, \ldots, s_{n}\right)$ and by Lemma 4.20 there is some closed $t^{\prime}=\tau\left(t_{1}^{\prime}, \ldots, t_{n}^{\prime}\right)$ with $s_{i} \preccurlyeq_{\text {cand }} t_{i}^{\prime}$ for all $i$ and $t^{\prime} \preccurlyeq_{l c c}^{o} t$.

- For the (nbeta)-reduction, $s=\left(\begin{array}{ll}s_{1} & s_{2}\end{array}\right)$, where $s_{1}=\left(\lambda x \cdot s_{1}^{\prime}\right), s_{2}$ is a closed term, and $t^{\prime}=\left(t_{1}^{\prime} t_{2}^{\prime}\right)$. The relation $\left(\lambda x . s_{1}^{\prime}\right)=s_{1} \preccurlyeq$ cand $t_{1}^{\prime}$ implies that there exists a closed expression $\lambda x . t_{1}^{\prime \prime} \preccurlyeq_{l c c}^{o} t_{1}^{\prime}$ with $s_{1}^{\prime} \preccurlyeq$ cand $t_{1}^{\prime \prime}$.

○ The first case is $t_{1}^{\prime} \stackrel{l c c, *}{\longrightarrow} c(\ldots)$ and $t_{1}^{\prime \prime} \in c B$ ot. Lemma 4.19 implies $\lambda x . s_{1}^{\prime} \preccurlyeq$ cand $\lambda x . t_{1}^{\prime \prime}$, and again by Lemma 4.19, we derive $s_{1}^{\prime}\left[s_{2} / x\right] \preccurlyeq$ cand $t_{1}^{\prime \prime}\left[s_{2} / x\right]$, where $t_{1}^{\prime \prime}\left[s_{2} / x\right] \in c B o t$. Then $t_{1}^{\prime \prime}\left[s_{2} / x\right] \preccurlyeq_{l c c}^{o} t$ by Lemma 4.14, which implies $s_{1}^{\prime}\left[s_{2} / x\right] \preccurlyeq$ cand $t$. Since $s \stackrel{l c c}{\longrightarrow} s_{1}^{\prime}\left[s_{2} / x\right]$, the lemma is proven for this case. 
$\circ$ The second case is $t_{1}^{\prime} \stackrel{l c c, *}{\longrightarrow} \lambda x \cdot t_{1}^{\prime \prime \prime}$ with $t_{1}^{\prime \prime} \preccurlyeq_{l c c}^{o} t_{1}^{\prime \prime \prime}$. We also obtain $\lambda x \cdot t_{1}^{\prime \prime} \preccurlyeq_{l c c}^{o} \lambda x \cdot t_{1}^{\prime \prime \prime}$, and by the properties of $\preccurlyeq_{l c c}^{o}$ w.r.t. reduction, also $t_{1}^{\prime \prime}\left[t_{2}^{\prime} / x\right] \preccurlyeq_{l c c}^{o} t_{1}^{\prime \prime \prime}\left[t_{2}^{\prime} / x\right]$. From $t^{\prime} \stackrel{l c c, *}{\longrightarrow} t_{1}^{\prime \prime \prime}\left[t_{2}^{\prime} / x\right]$ we obtain $t_{1}^{\prime \prime \prime}\left[t_{2}^{\prime} / x\right] \preccurlyeq l c c t$. Lemma 4.19 and transitivity of $\preccurlyeq_{l c c}$ now show $s_{1}^{\prime}\left[s_{2} / x\right] \preccurlyeq$ cand $t_{1}^{\prime \prime}\left[t_{2}^{\prime} / x\right]$. Hence $s_{1}^{\prime}\left[s_{2} / x\right] \preccurlyeq$ cand $t$, again using Lemma 4.19,

- Similar arguments as for the second case apply to the case-reduction.

- Suppose, the reduction is a (nseq)-reduction. Then $s \preccurlyeq$ cand $t$ and $s=\left(\right.$ seq $\left.s_{1} s_{2}\right)$. Lemma 4.20 implies that there is some closed (seq $\left.t_{1}^{\prime} t_{2}^{\prime}\right) \preccurlyeq_{l c c}^{o} t$ with $s_{i} \preccurlyeq c a n d t_{i}^{\prime}$. Since $s_{1}$ is a value, Lemma 4.23 shows that there is a reduction $t_{1}^{\prime} \stackrel{l c c, *}{\longrightarrow} t_{1}^{\prime \prime}$, where $t_{1}^{\prime \prime}$ is a value. There are the reductions $s \stackrel{l c c}{\longrightarrow} s_{2}$ and $\left(\right.$ seq $\left.t_{1}^{\prime} t_{2}^{\prime}\right) \stackrel{l c c, *}{\longrightarrow}\left(\right.$ seq $\left.t_{1}^{\prime \prime} t_{2}^{\prime}\right) \stackrel{l c c}{\longrightarrow} t_{2}^{\prime}$. Since $t_{2}^{\prime} \preccurlyeq_{l c c}^{o}\left(\right.$ seq $\left.t_{1}^{\prime} t_{2}^{\prime}\right) \preccurlyeq_{l c c}^{o} t$, and $s_{2} \preccurlyeq$ cand $t_{2}^{\prime}$, we obtain $s_{2} \preccurlyeq$ cand $t$.

Proposition 4.25. Let $s, t$ be closed expressions, $s \preccurlyeq$ cand $t$ and $s \stackrel{l c c}{\longrightarrow} s^{\prime}$. Then $s^{\prime} \preccurlyeq c a n d t$.

Proof. We use induction on the length of the path to the redex. The base case is proven in Proposition 4.24 . Let $R[s], t$ be closed, $R[s] \preccurlyeq{ }_{c a n d} t$ and $R[s] \stackrel{l c c}{\longrightarrow} R\left[s^{\prime}\right]$, where we assume that the redex $s$ is not at the top level and that $R$ is an $L_{l c c}$-reduction context. The relation $R[s] \preccurlyeq$ cand $t$ implies that $R[s]=\tau\left(s_{1}, \ldots, s_{n}\right)$ and that there is some closed expression $t^{\prime}$ with $t^{\prime}=\tau\left(t_{1}^{\prime}, \ldots, t_{n}^{\prime}\right) \preccurlyeq_{l c c}^{o} t$ with $s_{i} \preccurlyeq$ cand $t_{i}^{\prime}$ for all $i$. If $s_{j} \stackrel{l c c}{\longrightarrow} s_{j}^{\prime}$, then by induction hypothesis $s_{j}^{\prime} \preccurlyeq$ cand $t_{j}^{\prime}$. Since $\preccurlyeq_{\text {cand }}$ is operator-respecting, we also obtain $R\left[s^{\prime}\right]=\tau\left(s_{1}, \ldots, s_{j-1}, s_{j}^{\prime}, s_{j+1}, \ldots, s_{n}\right) \preccurlyeq$ cand $\tau\left(t_{1}^{\prime}, \ldots, t_{j-1}^{\prime}, t_{j}^{\prime}, t_{j+1}^{\prime}, \ldots, t_{n}^{\prime}\right)$, and from $\tau\left(t_{1}^{\prime}, \ldots, t_{n}^{\prime}\right) \preccurlyeq_{l c c}^{o} t$ we have $R\left[s^{\prime}\right]=\tau\left(s_{1}, \ldots, s_{j-1}, s_{j}^{\prime}, s_{j+1}, \ldots, s_{n}\right) \preccurlyeq_{\text {cand }} t$.

Lemma 4.26. If $\lambda x . s, \lambda x . t$ are closed, $\lambda x . s \preccurlyeq$ cand $\lambda x$.t, and $t \in c B o t$, then also $s \in c$ Bot.

Proof. For any closed $r$, we also have $(\lambda x . s) r \preccurlyeq$ cand $(\lambda x . t) r$, since $\preccurlyeq$ cand is operatorrespecting. From $t \in c B o t$, we obtain that $((\lambda x . t) r) \Uparrow_{l c c}$. Now suppose that $(\lambda x . s) r \stackrel{l c c, *}{\longrightarrow} s^{\prime}$, where $s^{\prime}$ is a value. Lemma 4.25 implies that $s^{\prime} \preccurlyeq$ cand $(\lambda x . t) r$. Now Lemma 4.23 shows that this is impossible. Hence $s \in c B o t$.

Now we can prove an improvement of Lemma 4.23,

Lemma 4.27. Let $s, t$ be closed expressions such that $s=\theta\left(s_{1}, \ldots, s_{n}\right)$ is a value and $s \preccurlyeq$ cand $t$. Then there are two possibilities:

(1) $s=\lambda x . s_{1}, t \downarrow_{l c c} c\left(t_{1}, \ldots, t_{n}\right)$ where $c$ is a constructor, and $s_{1} \in c B o t$.

(2) there is some closed value $t^{\prime}=\theta\left(t_{1}, \ldots, t_{n}\right)$ with $t \stackrel{l c c, *}{\longrightarrow} t^{\prime}$ and for all $i: s_{i} \preccurlyeq$ cand $t_{i}$.

Proof. This follows from Lemma 4.23 and Lemma 4.26.

Now we are ready to prove that the precongruence candidate and similarity coincide.

Theorem 4.28. $\left(\preccurlyeq_{\text {cand }}\right)^{c}=\preccurlyeq_{l c c}$ and $\preccurlyeq_{\text {cand }}=\preccurlyeq_{l c c}^{o}$.

Proof. Since $\preccurlyeq_{l c c} \subseteq\left(\preccurlyeq_{\text {cand }}\right)^{c}$ by Lemma 4.19, we have to show that $\left(\preccurlyeq_{\text {cand }}\right)^{c} \subseteq \preccurlyeq l c c$. Therefore it is sufficient to show that $(\preccurlyeq \text { cand })^{c}$ satisfies the fixpoint equation for $\preccurlyeq l c c$. We show that $\left(\preccurlyeq_{\text {cand }}\right)^{c} \subseteq F_{l c c}\left(\left(\preccurlyeq_{\text {cand }}\right)^{c}\right)$. Let $s\left(\preccurlyeq_{\text {cand }}\right)^{c} t$ for closed terms $s, t$. We show that $s \quad F_{l c c}\left(\left(\preccurlyeq_{\text {cand }}\right)^{c}\right) t$ : If $s \Uparrow_{l c c}$, then $s \quad F_{l c c}\left(\left(\preccurlyeq_{c a n d}\right)^{c}\right) t$ holds by Definition 4.10. If $s \downarrow_{l c c} \theta\left(s_{1}, \ldots, s_{n}\right)$, then $\theta\left(s_{1}, \ldots, s_{n}\right)\left(\preccurlyeq r_{c a n d}\right)^{c} t$ by Proposition 4.25 .

Lemmas 4.25 and 4.27 show that there are two possibilities:

- $t \stackrel{l c c, *}{\longrightarrow} c\left(t_{1}, \ldots, t_{n}\right)$ for a constructor $c, s \downarrow_{l c c} \lambda x . s_{1}$, and $s_{1} \in c B o t$. 
- $t \stackrel{l c c, *}{\longrightarrow} \theta\left(t_{1}, \ldots, t_{n}\right)$ and for all $i: s_{i} \preccurlyeq c a n d ~ t_{i}$.

This implies $s F_{l c c}\left((\preccurlyeq c a n d) c\right.$. $t$. Thus the fixpoint property of $\left(\preccurlyeq_{c a n d}\right)^{c}$ w.r.t. $F_{l c c}$ holds, and hence $\left(\preccurlyeq_{c a n d}\right)^{c}=\preccurlyeq l c c$.

Now we prove the second part. The first part, $\left(\preccurlyeq_{\text {cand }}\right)^{c} \subseteq \preccurlyeq_{l c c}$, implies $\left(\left(\preccurlyeq_{\text {cand }}\right)^{c}\right)^{o} \subseteq$ $\preccurlyeq_{l c c}^{o}$ by monotonicity. Lemma 4.19 (77) implies $\preccurlyeq_{c a n d} \subseteq\left(\left(\preccurlyeq_{c a n d}\right)^{c}\right)^{o} \subseteq \preccurlyeq_{l c c}^{o}$. The other direction is proven in Lemma 4.19 (3).

Since $\preccurlyeq_{l c c}^{o}$ is reflexive and transitive (Lemma 4.15) and $\left(\preccurlyeq_{c a n d}\right)^{c}$ is operator-respecting (Lemma 4.19 (2)), this immediately implies:

Corollary 4.29. $\preccurlyeq_{l c c}^{o}$ is a precongruence on expressions $\mathbb{E}_{\lambda}$. If $\sigma$ is a substitution, then $s \preccurlyeq_{l c c}^{o} t$ implies $\sigma(s) \preccurlyeq_{l c c}^{o} \sigma(t)$.

Lemma 4.30. $\preccurlyeq_{l c c}^{o} \subseteq \leq_{l c c}$.

Proof. Let $s, t$ be expressions with $s \preccurlyeq_{l c c}^{o} t$ such that $C[s] \downarrow_{l c c}$. Let $\sigma$ be a substitution that replaces all free variables of $C[s], C[t]$ by $\Omega$. The properties of the call-by-name reduction show that also $\sigma(C[s]) \downarrow_{l c c}$. Since $\sigma(C[s])=\sigma(C)[\sigma(s)], \sigma(C[t])=\sigma(C)[\sigma(t)]$ and since $\sigma(s) \preccurlyeq_{l c c}^{o} \sigma(t)$, we obtain from the precongruence property of $\preccurlyeq_{l c c}^{o}$ that also $\sigma(C[s]) \preccurlyeq l c c$ $\sigma(C[t])$. Hence $\sigma(C[t]) \downarrow_{l c c}$. This is equivalent to $C[t] \downarrow_{l c c}$, since free variables are replaced by $\Omega$, and thus they cannot overlap with redexes. Hence $\preccurlyeq_{l c c}^{o} \subseteq \leq_{l c c}$.

Corollary 4.31. $s \stackrel{l c c}{\longrightarrow} s^{\prime}$ implies $s \sim_{l c c} s^{\prime}$. Thus the reduction rules of the calculus $L_{l c c}$ are correct w.r.t. $\sim_{l c c}$ in any context.

Proof. This follows from Lemmas 4.21 and 4.30 .

Now we show a characterization for $\mathbb{E}_{\lambda}$-expressions, which includes the previously mentioned irregularity of $\leq_{l c c}$ :

Proposition 4.32. Let $s$ be a closed $L_{l c c}$-expression. Then there are three cases: $s \sim_{l c c} \Omega$, $s \sim_{l c c} \lambda x . s^{\prime}$ for some $s^{\prime}, s \sim_{l c c}\left(c s_{1} \ldots s_{n}\right)$ for some terms $s_{1}, \ldots, s_{n}$ and constructor $c$.

For two closed $L_{l c c}$-expressions $s, t$ with $s \leq_{l c c} t$ : Either $s \sim_{l c c} \Omega$, or $s \sim_{l c c}\left(c s_{1} \ldots s_{n}\right)$, $t \sim_{l c c}\left(c t_{1} \ldots t_{n}\right)$ and $s_{i} \leq_{l c c} t_{i}$ for all $i$ for some terms $s_{1}, \ldots, s_{n}, t_{1}, \ldots, t_{n}$ and constructor $c$, or $s \sim_{l c c} \lambda x . s^{\prime}$ and $t \sim_{l c c} \lambda x . t^{\prime}$ for some expressions $s^{\prime}, t^{\prime}$ with $s^{\prime} \leq_{l c c}^{o} t^{\prime}$, or $s \sim_{l c c} \lambda x . s^{\prime}$ and $t \sim_{l c c}\left(c t_{1} \ldots t_{n}\right)$ for some term $s^{\prime} \in c B$ ot, expressions $t_{1}, \ldots, t_{n}$ and constructor $c$.

Proof. We apply Lemma 4.30, Corollary 4.31 then shows that using reduction the classification of closed expressions into the classes w.r.t. $\sim_{l c c}$ holds.

For two closed $L_{l c c}$-expressions $s, t$ with $s \preccurlyeq l c c t$ : we obtain the classification in the lemma but with $\preccurlyeq_{l c c}$ instead of $\leq_{l c c}$. For the three cases $s \simeq_{l c c} \Omega$, both $s, t$ are equivalent to constructor expressions, and both $s, t$ are equivalent to abstractions, we obtain also that $s \leq_{l c c} t$. In the last case $\lambda x . s^{\prime} \preccurlyeq_{l c c}\left(c s_{1} \ldots s_{n}\right)$, we also obtain from the $\preccurlyeq_{l c c}$-definition, that it is valid and from Lemma 4.30, that it implies $\lambda x . s^{\prime} \leq_{l c c}\left(c s_{1} \ldots s_{n}\right)$. Other combinations of constructor applications, abstractions and $\Omega$ cannot be in $\leq_{l c c}$-relation:

- $\left(c t_{1} \ldots t_{n}\right) \mathbb{Z}_{l c c} \Omega$ and $\lambda x . s \mathbb{Z}_{l c c} \Omega$ since the empty context distinguishes them.

- $\left(c_{1} s_{1} \ldots s_{n}\right) \not_{l c c}\left(c_{2} t_{1} \ldots t_{m}\right)$ : Let $C:=\operatorname{case}_{T}[\cdot]\left(c_{1} x_{1} \ldots x_{n} \rightarrow \lambda y . y\right)$ alts where all alternatives in alts have right hand side $\Omega$. Then $C\left[\left(c_{1} s_{1} \ldots s_{n}\right)\right] \downarrow_{l c c}$ but $C\left[\left(c_{2} t_{1} \ldots t_{m}\right)\right] \Uparrow_{l c c}$.

- $\left(c s_{1} \ldots s_{n}\right) \mathbb{Z}_{l c c}\left(c t_{1} \ldots t_{n}\right)$ if $s_{i} \mathbb{Z}_{l c c} t_{i}$ : Let context $D$ be the witness for $s_{i} \mathbb{Z}_{l c c} t_{i}$. Then $C=\operatorname{case}_{T}[\cdot]\left(c x_{1} \ldots x_{n} \rightarrow D\left[x_{i}\right]\right)$ distinguishes $\left(c s_{1} \ldots s_{n}\right)$ and $\left(c t_{1} \ldots t_{n}\right)$

- $\left(c s_{1} \ldots s_{n}\right) \mathbb{Z}_{l c c}(\lambda x . t)$ : The context $\operatorname{case}_{T}[\cdot]\left(c x_{1} \ldots x_{n} \rightarrow \lambda y . y\right)$ alts is a witness. 
- $\lambda x . s \mathbb{Z}_{l c c} \lambda x . t$ if $s \mathbb{Z}_{l c c} t$ : Let $D$ be the witness for $s \mathbb{L}_{l c c} t$. Then $C=D[([\cdot] x)]$ distinguishes $\lambda$ x.s and $\lambda$ x.t.

- $\lambda x . s \mathbb{E}_{l c c}\left(c t_{1} \ldots t_{n}\right)$ if $s \notin c B o t$ : Since $s \notin c B o t$ and $F V(s) \subseteq\{x\}$, there exists a closing substitution $\sigma=\{x \mapsto r\}$ such that $\sigma(s) \downarrow_{l c c}$. For the context $C=([\cdot] r)$ the expression $C[\lambda x . s]$ converges while $C\left[\left(c t_{1} \ldots t_{n}\right)\right]$ diverges.

Lemma 4.33. $\leq_{l c c} \subseteq \preccurlyeq_{l c c}^{o}$.

Proof. The relation $\leq_{l c c}^{c}$ satisfies the fixpoint condition, i.e. $\leq_{l c c}^{c} \subseteq F_{l c c}\left(\leq_{l c c}^{c}\right)$, which follows from Corollary 4.31 and Proposition 4.32 .

Lemmas 4.30 and 4.33 immediately imply:

Theorem 4.34. $\preccurlyeq_{l c c}^{o}=\leq_{l c c}$.

4.2.2. Alternative Definitions of Bisimilarity in $L_{l c c}$. We want to analyze the translations between our calculi, and the inherent contextual equivalence. This will require to show that several differently defined relations are all equal to contextual equivalence.

Using Theorem 4.8 we show that in $L_{l c c}$, behavioral equivalence can also be proved inductively:

Definition 4.35. The set $Q_{l c c}$ of contexts $Q$ is assumed to consist of the following contexts:

(i) $([\cdot] r)$ for all closed $r$,

(ii) for all types $T$, constructors $c$ of $T$, and indices $i$ :

$\left(\operatorname{case}_{T}[\cdot]\right.$ of $\left.\ldots\left(c x_{1} \ldots x_{\operatorname{ar}(c)} \rightarrow x_{i}\right) \ldots\right)$ where all right hand sides of other casealternatives are $\Omega$,

(iii) for all types $T$ and constructors $c$ of $T$ : $\left(\operatorname{case}_{T}[\cdot]\right.$ of $\left.\ldots\left(c x_{1} \ldots x_{\operatorname{ar}(c)} \rightarrow \operatorname{True}\right) \ldots\right)$ where all right hand sides of other case-alternatives are $\Omega$.

The relations $\preccurlyeq_{l c c, Q_{l c c}}$, $\leq_{l c c, Q_{l c c}}$ are instantiations of Definitions 2.5 and Definition 2.6, respectively, with the set $Q_{l c c}$ and the closed part of $L_{l c c}$ consisting of the subsets of all closed $\mathbb{E}_{\lambda}$-expressions, closed contexts $\mathbb{C}_{\lambda}$, and closed answers $\mathbb{A}_{l c c}$.

Lemma 4.36. The calculus $L_{l c c}$ is convergence-admissible in the sense of Definition 4.5 , where the $Q$-contexts are defined as above.

Proof. Values in $L_{l c c}$ are $L_{l c c}$-WHNFs. The contexts $Q$ are reduction contexts in $L_{l c c}$. Hence every reduction of $Q[s]$ will first evaluate $s$ to $v$ and then evaluate $Q[v]$.

Theorem 4.37. $\preccurlyeq_{l c c}=\preccurlyeq_{l c c, Q_{l c c}}=\leq_{l c c, Q_{l c c}}$ and $\preccurlyeq_{l c c}^{o}=\preccurlyeq_{l c c, Q_{l c c}}^{o}=\leq_{l c c, Q_{l c c}}^{o}$

Proof. Theorem 4.8. shows $\preccurlyeq_{l c c, Q_{l c c}}=\leq_{l c c, Q_{l c c}}$ since $L_{l c c}$ is convergence-admissible.

The first equation is proved by showing that the relations satisfy the fixpoint equations of the other one in Definition 4.10 and 2.5. respectively.

- $\preccurlyeq_{l c c} \subseteq F_{Q}\left(\preccurlyeq_{l c c}\right)$ : Assume $s \preccurlyeq_{l c c} t$ for two closed $s, t$. If $s \downarrow_{l c c} v$, then $t \downarrow_{l c c} w$ for values $v, w$. Since reduction preserves $\simeq_{l c c}$, the fixpoint operator conditions are satisfied if $v, w$ are both abstractions or both constructor applications. If $v=\lambda x . s^{\prime}$ with $s^{\prime} \in c B o t$ and $w=c\left(t_{1}, \ldots, t_{n}\right), Q(v)$ diverges for all $Q$, hence $s F_{Q}\left(\preccurlyeq_{l c c}\right) t$.

- $\preccurlyeq_{l c c, Q_{l c c}} \subseteq F_{l c c}\left(\preccurlyeq_{l c c, Q_{l c c}}\right)$ : Assume $s \preccurlyeq_{l c c, Q_{l c c}} t$. Let $s \downarrow_{l c c} v$. Then also $t \downarrow_{l c c} w$ for some value $w$. In the cases that $v, w$ are both abstractions or both constructor applications, when using appropriate $Q$ of kind (ii) or (iii), the $F_{l c c}$-conditions are satisfied. If $v=\lambda x . s^{\prime}$ and $w=c\left(t_{1}, \ldots, t_{n}\right)$, we have to show that $s^{\prime} \in c B$ ot: this can be done using all $Q$-contexts of the form $([] r)$, since $(w r) \Uparrow_{l c c}$ in any case. 
Definition 4.38. Let $C E_{l c c}$ be the following set of closed $\mathbb{E}_{\lambda}$-expressions built from constructors, $\Omega$, and closed abstractions. These can be constructed according to the grammar:

$$
r \in C E_{l c c}::=\Omega|\lambda x . s|\left(c r_{1} \ldots r_{\operatorname{ar}(c)}\right)
$$

where $\lambda x . s$ is any closed $\mathbb{E}_{\lambda \text {-expression. }}$

The set $Q_{C E}$ is defined like the set $Q_{l c c}$ in Definition 4.35, but only expressions $r$ from $C E_{l c c}$ are taken into account in the contexts ([·] $\left.r\right)$ in (i).

We summarize several alternative definitions for contextual preorder and applicative simulation for $L_{l c c}$, where we also include further alternatives. (1) is contextual preorder, (2) the applicative simulation, (3), (4) and (5) are similar to the usual call-by-value variants, where (44) and (5) separate the closing part of contexts, where (5) can be seen as bridging the gap between call-by-need and call-by-name. (7) is the $\mathcal{Q}$-similarity, (8) is a further improved inductive $\mathcal{Q}$-simulation by restricting the set of test arguments for abstractions, and (9) is the co-inductive version of (8) .

Theorem 4.39. In $L_{l c c}$, all the following relations on open $\mathbb{E}_{\lambda}$-expressions are identical:

(1) $\leq_{l c c}$.

(2) $\preccurlyeq_{l c c}^{o}$.

(3) The relation $\leq_{l c c, 1}$ defined by $s_{1} \leq_{l c c, 1} s_{2}$ iff for all closing contexts $C: C\left[s_{1}\right] \downarrow_{l c c} \Longrightarrow$ $C\left[s_{2}\right] \downarrow_{l c c}$.

(4) The relation $\leq_{l c c, 2}$, defined as: $s_{1} \leq_{l c c, 2} s_{2}$ iff for all closed contexts $C$ and all closing substitutions $\sigma: C\left[\sigma\left(s_{1}\right)\right] \downarrow_{l c c} \Longrightarrow C\left[\sigma\left(s_{2}\right)\right] \downarrow_{l c c}$.

(5) The relation $\leq_{l c c, 3}$, defined as: $s_{1} \leq_{l c c, 3} s_{2}$ iff for all multi-contexts $M[\cdot, \ldots, \cdot]$ and all substitutions $\sigma: M\left[\sigma\left(s_{1}\right), \ldots, \sigma\left(s_{1}\right)\right] \downarrow_{l c c} \Longrightarrow M\left[\sigma\left(s_{2}\right), \ldots, \sigma\left(s_{2}\right)\right] \downarrow_{l c c}$.

(6) The relation $\leq_{l c c, 4}$, defined as: $s_{1} \leq_{l c c, 4} s_{2}$ iff for all contexts $C[\cdot]$ and all substitutions $\sigma: C\left[\sigma\left(s_{1}\right)\right] \downarrow_{l c c} \Longrightarrow C\left[\sigma\left(s_{2}\right)\right] \downarrow_{l c c}$.

(7) $\leq_{l c c, Q_{l c c}}^{o}$

(8) The relation $\leq_{l c c, Q_{C E}}$ where $\leq_{l c c, Q_{C E}}$ is defined as in Definition 2.6 instantiated by the closed part of $L_{l c c}$ and by the set $Q_{C E}$ in Definition 4.38 .

(9) The relation $\preccurlyeq_{l c c, Q_{C E}}$ is defined as in Definition 2.5 instantiated by the closed part of $L_{l c c}$ and by set $Q_{C E}$ in Definition 4.38 .

Proof. • (11) $\Longleftrightarrow$ (2) $\Longleftrightarrow$ (7): This is Theorem 4.34 and Theorem 4.37

- (11) $\Longleftrightarrow$ (3): The " $\Rightarrow$ "-direction is obvious. For the other direction let $s_{1} \leq_{l c c, 1} s_{2}$ and let $C$ be a context such that $\emptyset \neq F V\left(C\left[s_{1}\right]\right) \cup F V\left(C\left[s_{2}\right]\right)=\left\{x_{1}, \ldots, x_{n}\right\}$ and let $C\left[s_{1}\right] \downarrow_{l c c}$, i.e. $C\left[s_{1}\right] \stackrel{l c c, *}{\longrightarrow} v$ where $v$ is an abstraction or a constructor application. Let $C^{\prime}=\left(\lambda x_{1}, \ldots, x_{n} . C\right) \underbrace{\Omega \ldots \Omega}_{n \text {-times }}$. Then $C^{\prime}\left[s_{i}\right] \stackrel{l c c, \text { nbeta,* }}{\longrightarrow} s_{i}^{\prime}=C\left[s_{i}\right]\left[\Omega / x_{1}, \ldots, \Omega / x_{n}\right]$ for $i=1,2$. It is easy to verify that the reduction for $C\left[s_{1}\right]$ can also be performed for $s_{i}^{\prime}$, since no reduction in the sequence $C\left[s_{1}\right] \stackrel{l c c, *}{\longrightarrow} v$ can be of the form $R\left[x_{i}\right]$ with $R$ being a reduction context. Thus $C^{\prime}\left[s_{i}\right] \downarrow_{l c c}$. Since $C^{\prime}\left[s_{i}\right]$ must be closed for $i=1,2$, the precondition implies $C^{\prime}\left[s_{2}\right] \downarrow_{l c c}$ and also $s_{2}^{\prime} \downarrow_{l c c}$. W.l.o.g. let $s_{2}^{\prime} \stackrel{l c c, *}{\longrightarrow} v^{\prime}$ where $v^{\prime}$ is an $L_{l c c}$-WHNF. It is easy to verify that no term in this sequence can be of the form $R[\Omega]$, where $R$ is a reduction context, since otherwise the reduction would not terminate (since $R[\Omega] \stackrel{l c c,+}{\longrightarrow} R[\Omega])$. This implies that we can replace the $\Omega$-expression by the free variables, 
i.e. that $C\left[s_{2}\right] \downarrow_{l c c}$. Note that this also shows by the previous items (and Corollary 4.31) that (nbeta) is correct for $\sim_{l c c}$.

- (11) $\Longleftrightarrow$ (4): This follows from Corollary 4.31 since closing substitutions can be simulated by a context with subsequent (nbeta)-reduction. This also implies that (nbeta) is correct for $\sim_{l c c, 2}$ and by the previous item it also correct for $\sim_{l c c, 1}$ (where $\sim_{l c c_{i}}=\leq_{l c c, i} \cap \geq_{l c c, i}$ ). - (16) $\Longleftrightarrow$ (10) The direction " $\Longrightarrow$ " is trivial. For the other direction let $s_{1} \leq_{l c c} s_{2}$ and let $C$ be a context, $\sigma$ be a substitution, such that $C\left[\sigma\left(s_{1}\right)\right] \downarrow_{l c c}$. Let $\sigma=\left\{x_{1} \rightarrow t_{1}, \ldots x_{n} \rightarrow t_{n}\right\}$ and let $C^{\prime}=C\left[\left(\lambda x_{1}, \ldots, x_{n} \cdot[\cdot]\right) t_{1} \ldots t_{n}\right]$. Then $C^{\prime}\left[s_{1}\right] \stackrel{\text { nbeta, } n}{\longrightarrow} C\left[\sigma\left(s_{1}\right)\right]$. Since (nbeta)reduction is correct for $\sim_{l c c}$, we have $C^{\prime}\left[s_{1}\right] \downarrow_{l c c}$. Applying $s_{1} \leq_{l c c} s_{2}$ yields $C^{\prime}\left[s_{2}\right] \downarrow_{l c c}$. Since $C^{\prime}\left[s_{2}\right] \stackrel{\text { nbeta, } n}{\longrightarrow} C\left[\sigma\left(s_{2}\right)\right]$ and (nbeta) is correct for $\sim_{l c c}$, we have $C\left[\sigma\left(s_{2}\right)\right] \downarrow_{l c c}$.

- (15) $\Longleftrightarrow$ (6) : Obviously, $s_{1} \leq_{l c c, 3} s_{2} \Longrightarrow s_{1} \leq_{l c c, 4} s_{2}$. We show the other direction by

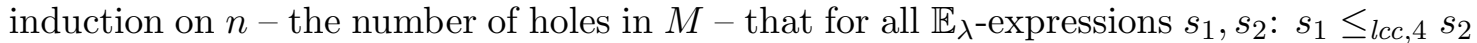
implies $M\left[\sigma\left(s_{1}\right), \ldots \sigma\left(s_{1}\right)\right] \downarrow_{l c c} \Longrightarrow M\left[\sigma\left(s_{2}\right), \ldots \sigma\left(s_{2}\right)\right] \downarrow_{l c c}$.

The base cases for $n=0,1$ are obvious. For the induction step assume that $M$ has $n>1$ holes. Let $M^{\prime}=M\left[\sigma\left(s_{1}\right), \cdot_{2}, \ldots,{ }_{n}\right]$ and $M^{\prime \prime}=M\left[\sigma\left(s_{2}\right), \cdot_{2}, \ldots, \cdot_{n}\right]$. Then obviously $M^{\prime}\left[\sigma\left(s_{1}\right), \ldots, \sigma\left(s_{1}\right)\right]=M\left[\sigma\left(s_{1}\right), \ldots, \sigma\left(s_{1}\right)\right]$ and thus $M^{\prime}\left[\sigma\left(s_{1}\right), \ldots, \sigma\left(s_{1}\right)\right] \downarrow_{l c c}$. For $C=M\left[\cdot_{1}, \sigma\left(s_{1}\right), \ldots, \sigma\left(s_{1}\right)\right]$ we have $C\left[\sigma\left(s_{1}\right)\right]=M^{\prime}\left[\sigma\left(s_{1}\right), \ldots, \sigma\left(s_{1}\right)\right]$ and also $C\left[\sigma\left(s_{2}\right)\right]=$ $M^{\prime \prime}\left[\sigma\left(s_{1}\right), \ldots, \sigma\left(s_{1}\right)\right]$. Since $C\left[\sigma\left(s_{1}\right)\right] \downarrow_{l c c}$, the relation $s_{1} \leq_{l c c, 4} s_{2}$ implies that $C\left[\sigma\left(s_{2}\right)\right] \downarrow_{l c c}$ and hence $M^{\prime \prime}\left[\sigma\left(s_{1}\right), \ldots, \sigma\left(s_{1}\right)\right] \downarrow_{l c c}$. Now, since the number of holes of $M^{\prime \prime}$ is strictly smaller than $n$, the induction hypothesis show that $M^{\prime \prime}\left[\sigma\left(s_{2}\right), \ldots, \sigma\left(s_{2}\right)\right] \downarrow_{l c c}$. Because of $M^{\prime \prime}\left[\sigma\left(s_{2}\right), \ldots, \sigma\left(s_{2}\right)\right]=M\left[\sigma\left(s_{2}\right), \ldots, \sigma\left(s_{2}\right)\right]$ we have $M\left[\sigma\left(s_{2}\right), \ldots, \sigma\left(s_{2}\right)\right] \downarrow_{l c c}$.

- (17) $\Longleftrightarrow$ (8): The direction (77) $\Longrightarrow$ (8) is trivial.

For the other direction we show that $\leq_{l c c, Q_{C E}}^{o} \subseteq \leq_{l c c, Q_{l c c}}^{o}$ by showing that the inclusion $\leq_{l c c, Q_{C E}} \subseteq \leq_{l c c, Q_{l c c}}$ holds. Let $s_{1}, s_{2}$ be closed expressions with $s_{1} \leq_{l c c, Q_{C E}} s_{2}$ and let $Q_{1}\left[\ldots Q_{n}\left[s_{1}\right] \ldots\right] \downarrow_{l c c}$ for $Q_{i} \in Q_{l c c}$. Let $m$ be the number of normal-order-reductions of $Q_{1}\left[\ldots Q_{n}\left[s_{1}\right] \ldots\right]$ to an $L_{l c c}$-WHNF. Since the reduction rules are correct w.r.t. $\sim_{l c c}$, for every subexpression $r$ of the contexts $Q_{i}$, there is some $r^{\prime}$ with $r^{\prime} \leq_{l c c} r$, where $r^{\prime} \in Q_{C E}$, which is derived from $r$ by (top-down)-reduction, which may also be non-normal order, i.e. $r \stackrel{l c c, *}{\longrightarrow} r_{m+1}$ where $r_{m+1}$ has reducible subexpressions (not in an abstraction) only at depth at least $m+1$. All those deep subexpressions are then replaced by $\Omega$, and this construction results in $r^{\prime}$. By construction, $r^{\prime} \leq_{l c c} r$. We do this for all the contexts $Q_{i}$, and obtain thus contexts $Q_{i}^{\prime}$. The construction using the depth $m$ shows that $\left(Q_{1}^{\prime}\left[\ldots\left[Q_{n}^{\prime}\left[s_{1}\right]\right]\right]\right) \downarrow_{l c c}$, since the normal-order reduction does not use subexpressions at depth greater than $m$ in those $r^{\prime}$. By assumption, this implies $\left(Q_{1}^{\prime}\left[\ldots\left[Q_{n}^{\prime}\left[s_{2}\right]\right]\right]\right) \downarrow_{l c c}$, and since $\left(Q_{1}^{\prime}\left[\ldots\left[Q_{n}^{\prime}\left[s_{2}\right]\right]\right]\right) \leq_{l c c}\left(Q_{1}^{\prime}\left[\ldots\left[Q_{n}^{\prime}\left[s_{2}\right]\right]\right]\right)$, this also implies $\left(Q_{1}\left[\ldots\left[Q_{n}\left[s_{2}\right]\right]\right]\right)$.

- (8) $\Longleftrightarrow$ (9): This follows for the relations on closed expressions by Theorem 4.8, since the deterministic calculus (see Def. 2.1) for $L_{l c c}$ with $Q_{C E}$ as defined above is convergenceadmissible. It also holds for the extensions to open expressions, since the construction for the open extension is identical for both relations.

Also the following can easily be derived from Theorem 4.39 and Corollary 4.31,

Proposition 4.40. For open $\mathbb{E}_{\lambda}$-expressions $s_{1}, s_{2}$, where all free variables of $s_{1}, s_{2}$ are in $\left\{x_{1}, \ldots, x_{n}\right\}: s_{1} \leq_{l c c} s_{2} \Longleftrightarrow \lambda x_{1}, \ldots x_{n} . s_{1} \leq_{l c c} \lambda x_{1}, \ldots x_{n} . s_{2}$

Proposition 4.41. Given any two closed $\mathbb{E}_{\lambda}$-expressions $s_{1}, s_{2}: s_{1} \leq_{l c c} s_{2}$ iff the following conditions hold: 
- If $s_{1} \downarrow_{l c c} \lambda x . s_{1}^{\prime}$, then either (i) $s_{2} \downarrow_{l c c} \lambda x . s_{2}^{\prime}$, and for all (closed) $r \in C E_{l c c}: s_{1} r \leq_{l c c} s_{2} r$, or (ii) $s_{2} \downarrow_{l c c}\left(c s_{1}^{\prime \prime} \ldots s_{n}^{\prime \prime}\right)$ and $s_{1}^{\prime} \in c B o t$.

- if $s_{1} \downarrow_{l c c}\left(c s_{1}^{\prime} \ldots s_{n}^{\prime}\right)$, then $s_{2} \downarrow_{l c c}\left(c s_{1}^{\prime \prime} \ldots s_{n}^{\prime \prime}\right)$, and for all $i: s_{i}^{\prime} \leq_{l c c} s_{i}^{\prime \prime}$

Proof. The if-direction follows from the congruence property of $\leq_{l c c}$ and the correctness of reductions. The only-if direction follows from Theorem 4.39.

This immediately implies

Proposition 4.42. Given any two closed $\mathbb{E}_{\lambda}$-expressions $s_{1}, s_{2}$.

- If $s_{1}, s_{2}$ are abstractions, then $s_{1} \leq_{l c c} s_{2}$ iff for all closed $r \in C E_{l c c}$ : $s_{1} r \leq_{l c c} s_{2} r$

- If $s_{1}=\left(c t_{1} \ldots t_{n}\right)$ and $s_{2}=\left(c^{\prime} t_{1}^{\prime} \ldots t_{m}^{\prime}\right)$ are constructor expressions, then $s_{1} \leq_{l c c} s_{2}$ iff $c=c^{\prime}, n=m$ and for all $i: t_{i} \leq_{l c c} t_{i}^{\prime}$

We finally consider a more relaxed notion of similarity which allows to use known contextual equivalences as intermediate steps when proving similarity of expressions:

Definition 4.43 (Similarity up to $\sim_{l c c}$ ). Let $\preccurlyeq_{l c c, \sim}$ be the greatest fixpoint of the following operator $F_{l c c, \sim}$ on closed $L_{l c c}$-expressions:

We define an operator $F_{l c c, \sim}$ on binary relations $\eta$ on closed $L_{l c c}$-expressions: $s F_{l c c, \sim}(\eta) t$ iff the following holds:

(1) If $s \sim_{l c c} \lambda x . s^{\prime}$ then there are two possibilities: (i) if $t \sim_{l c c}\left(c t_{1} \ldots t_{n}\right)$ then $s^{\prime} \in c B o t$, or (ii) if $t \sim_{l c c} \lambda x . t^{\prime}$ then for all closed $r:\left(\left(\lambda x . s^{\prime}\right) r\right) \eta\left(\left(\lambda x . t^{\prime}\right) r\right)$;

(2) If $s \sim_{l c c}\left(c s_{1} \ldots s_{n}\right)$ then $t \sim_{l c c}\left(c t_{1} \ldots t_{n}\right)$ and $s_{i} \eta t_{i}$ for all $i$.

Obviously, we have $s \preccurlyeq l c c, \sim t$ iff one of the three cases holds: (i) $s \sim_{l c c} \lambda x . s^{\prime}, t \sim_{l c c} \lambda x . t^{\prime}$, and $\left(\lambda x . s^{\prime}\right) r \preccurlyeq_{l c c, \sim}\left(\lambda x . t^{\prime}\right) r$ for all closed $r$; (ii) $s \sim_{l c c} \lambda x . s^{\prime}, t \sim_{l c c}\left(c t_{1} \ldots t_{n}\right)$, and $s^{\prime} \in c B o t$, or (iii) $s \sim_{l c c}\left(c s_{1} \ldots s_{n}\right), t \sim_{l c c}\left(c t_{1} \ldots t_{n}\right)$, and $s_{i} \preccurlyeq l c c, \sim t_{i}$ for all $i$.

Proposition 4.44. $\preccurlyeq_{l c c, \sim}=\preccurlyeq_{l c c}=\leq_{l c c}^{c}$, and $\preccurlyeq_{l c c, \sim}^{o}=\preccurlyeq_{l c c}^{o}=\leq_{l c c}$.

Proof. We show the first equation via the fixpoint equations. (i) We prove that the relation $\preccurlyeq_{l c c, \sim}$ satisfies the fixpoint equation for $\preccurlyeq_{l c c}$ : Let $s \preccurlyeq_{l c c, \sim} t$, where $s, t$ are closed. If $s \downarrow_{l c c}\left(c s_{1} \ldots s_{n}\right)$, then also $s \sim_{l c c}\left(c s_{1} \ldots s_{n}\right)$ which clearly implies $t_{\downarrow_{l c c}}\left(c t_{1} \ldots t_{n}\right)$, and also $t \sim_{l c c}\left(c t_{1} \ldots t_{n}\right)$. The relation $\preccurlyeq l c c, \sim$ is a fixpoint of $F_{l c c, \sim}(\eta)$, hence $s_{i} \preccurlyeq l c c, \sim t_{i}$ for all $i$.

If $s \downarrow_{l c c} \lambda x . s^{\prime}$ and $t \downarrow_{l c c} \lambda x . t^{\prime}$ then similar arguments show $\left(\left(\lambda x . s^{\prime}\right) r\right) \preccurlyeq l c c, \sim\left(\left(\lambda x . t^{\prime}\right) r\right)$ for all $r$. If $s \downarrow_{l c c} \lambda x . s^{\prime}$ and $t \downarrow_{l c c}\left(c t_{1} \ldots t_{n}\right)$, then $s \sim_{l c c} \lambda x . s^{\prime}$ and $t \sim_{l c c}\left(c t_{1} \ldots t_{n}\right)$. Again the fixpoint property of $\preccurlyeq l c c, \sim$ shows $s^{\prime} \in c B o t$.

(ii) We prove that the relation $\preccurlyeq l c c$ satisfies the fixpoint equation for $F_{l c c, \sim}$ : Let $s \preccurlyeq l c c t$ for closed $s, t$. We know that this is the same as $s \leq_{l c c} t$. If $s \sim_{l c c}\left(c s_{1} \ldots s_{n}\right)$, then clearly

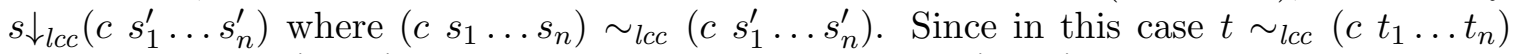
and thus $t_{l c c}\left(c t_{1}^{\prime} \ldots t_{n}^{\prime}\right)$ where $t \sim_{l c c}\left(c t_{1} \ldots t_{n}\right) \sim_{l c c}\left(c t_{1}^{\prime} \ldots t_{n}^{\prime}\right)$, and also $s_{i} \preccurlyeq l c c, \sim t_{i}$ for all $i$ holds, since reduction is correct. If $s \sim_{l c c} \lambda x . s^{\prime}$ and $s \sim_{l c c} \lambda x . t^{\prime}$ then $s \downarrow_{l c c} \lambda x . s^{\prime \prime}$ and $t_{l c c} \lambda x . t^{\prime \prime}$ and $\left(\left(\lambda x . s^{\prime}\right) r\right) \preccurlyeq_{l c c, \sim}\left(\left(\lambda x . t^{\prime}\right) r\right)$.

If $s \sim_{l c c} \lambda x . s^{\prime}$ and $s \sim_{l c c}\left(c t_{1} \ldots t_{n}\right)$, then for $s \downarrow_{l c c} \lambda x . s^{\prime \prime}$, we have $\lambda x . s^{\prime} \sim_{l c c} \lambda x . s^{\prime \prime}$, and since $s \leq_{l c c} t$, the characterization of expressions in Proposition 4.32 shows $s^{\prime}, s^{\prime \prime} \in c$ Bot. 


\section{The Translation $W: L_{L R} \rightarrow L_{\text {name }}$}

The translation $W: L_{L R} \rightarrow L_{\text {name }}$ is defined as the identity on expressions and contexts, but the definitions of convergence predicates are different. In this section we prove that contextual equivalence based on $L_{L R^{-}}$evaluation and contextual equivalence based on $L_{n a m e^{-}}$ evaluation are equivalent. We use infinite trees to connect both evaluation strategies. In [SS07] a similar result was shown for a lambda calculus without seq, case, and constructors.

5.1. Calculus for Infinite Trees $L_{\text {tree }}$. We define infinite expressions which are intended to be the letrec-unfolding of the $\mathbb{E}_{\mathcal{L}}$-expressions with the extra condition that cyclic variable chains lead to local nontermination represented by Bot. We then define the calculus $L_{\text {tree }}$ which has infinite expressions and performs reduction on infinite expressions.

Definition 5.1. Infinite expressions $\mathcal{E}_{\mathcal{I}}$ are defined like expressions $\mathbb{E}_{\mathcal{L}}$ without letrecexpressions, adding a constant Bot, and interpreting the grammar co-inductively, i.e. the grammar is as follows

$$
\begin{aligned}
S, T, S_{i}, T_{i} \in \mathcal{E}_{\mathcal{I}}::= & x\left|\left(S_{1} S_{2}\right)\right|(\lambda x . S) \mid \text { Bot } \\
& \left|\left(c S_{1} \ldots S_{\operatorname{ar}(c)}\right)\right|\left(\operatorname{seq} S_{1} S_{2}\right) \mid\left(\text { case }_{T} S \text { of alts }\right)
\end{aligned}
$$

In order to distinguish in the following the usual expressions from the infinite ones, we say tree or infinite expressions. As meta-symbols we use $s, s_{i}, t, t_{i}$ for finite expressions and $S, T, S_{i}, T_{i}$ for infinite expressions. The constant Bot is without any reduction rule.

In the following definition of a mapping from finite expressions to their infinite images, we sometimes use the explicit binary application operator @ for applications inside the trees (i.e. an application in the tree is sometimes written as (@ $\left.S_{1} S_{2}\right)$ instead of $\left(S_{1} S_{2}\right)$ ), since it is easier to explain, but use the common notation in other places. A position is a finite sequence of positive integers, where the empty position is denoted as $\varepsilon$. We use Dewey notation for positions, i.e. the position i.p is the sequence starting with $i$ followed by position $p$. For an infinite tree $S$ and position $p$, the notation $\left.S\right|_{p}$ means the subtree at position $p$ and $p(S)$ denotes the head symbol of $\left.S\right|_{p}$.

This induces the representation of an infinite expression $S$ as a (partial) function $S$ from positions to labels where application of the function $S$ to a position $p$ is written as $p(S)$ and where the labels are @, $\operatorname{case}_{T},\left(c x_{1} \ldots x_{n}\right)$ (for a case-alternative), seq, $c, \lambda x$, and $x$. The domain of such a function must be a prefix-closed set of positions, and the continuations of a position $p$ depend on the label at $p$ and must coincide with the syntax according to the grammar in Definition 5.1 .

Definition 5.2. The translation $I T: \mathbb{E}_{\mathcal{L}} \rightarrow \mathcal{E}_{\mathcal{I}}$ translates an expression $s \in \mathbb{E}_{\mathcal{L}}$ into its infinite tree $I T(s) \in \mathcal{E}_{\mathcal{I}}$. We define the mapping $I T$ by providing an algorithm that, computes the partial function $I T(s)$ from positions to labels. Given a position $p$, computing $p(I T(s))$ starts with $s \|_{p}$ and then proceeds with the rules given in Fig. 9. The first group of rules defines the computed label for the position $\varepsilon$, the second part of the rules describes the general case for positions. If the computation fails (or is undefined), then the position is not valid in the tree $I T(s)$. The equivalence of infinite expressions is extensional equality of the corresponding functions, where we additionally do not distinguish $\alpha$-equal trees.

Example 5.3. The expression letrec $x=x, y=(\lambda z . z) x y$ in $y$ has the corresponding tree $\left(\left(\lambda z_{1} . z_{1}\right)\right.$ Bot $\left(\left(\lambda z_{2} . z_{2}\right)\right.$ Bot $\left(\left(\lambda z_{3} . z_{3}\right)\right.$ Bot $\left.\left.\left.\ldots\right)\right)\right)$. 


$$
\begin{aligned}
C\left[(s t) \|_{\varepsilon}\right] & \mapsto @ \\
C\left[\left(\operatorname{case}_{T} \ldots\right) \|_{\varepsilon}\right] & \mapsto \operatorname{case}_{T} \\
C\left[\left(c x_{1} \ldots x_{n} \rightarrow s\right) \|_{\varepsilon}\right] & \mapsto\left(c x_{1} \ldots x_{n}\right) \quad \text { for a case-alternative } \\
C\left[(\operatorname{seq} s t) \|_{\varepsilon}\right] & \mapsto \text { seq } \\
C\left[\left(c s_{1} \ldots s_{n}\right) \|_{\varepsilon}\right] & \mapsto c \\
C\left[(\lambda x . s) \|_{\varepsilon}\right] & \mapsto \lambda x \\
C\left[x \|_{\varepsilon}\right] & \mapsto x \text { if } x \text { is a free variable or a lambda-bound variable in } C[x]
\end{aligned}
$$

The cases for general positions $p$ :

$$
\begin{aligned}
& \text { 1. } C\left[(\lambda x . s) \|_{1 . p}\right] \quad \mapsto C\left[\lambda x .\left(s \|_{p}\right)\right] \\
& \text { 2. } C\left[(s t) \|_{1 . p}\right] \quad \mapsto C\left[\left(s \|_{p} t\right)\right] \\
& \text { 3. } C\left[(s t) \|_{2 . p}\right] \quad \mapsto C\left[\left(s t \|_{p}\right)\right] \\
& \text { 4. } C\left[(\text { seq } s t) \|_{1 . p}\right] \quad \mapsto C\left[\left(\text { seq } s \|_{p} t\right)\right] \\
& \text { 5. } \quad C\left[(\operatorname{seq} s t) \|_{2 . p}\right] \quad \mapsto C\left[\left(\operatorname{seq} s t \|_{p}\right)\right]
\end{aligned}
$$

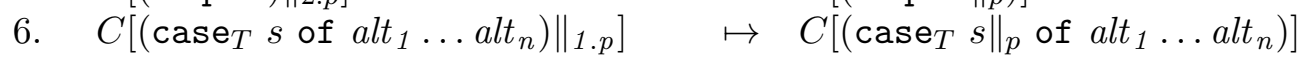

$$
\begin{aligned}
& \text { 7. } C\left[\left(\operatorname{case}_{T} s \text { of } \text { alt }_{1} \ldots \text { alt }_{n}\right) \|_{(i+1) . p}\right] \mapsto C\left[\left(\operatorname{case}_{T} s \text { of alt }{ }_{1} \ldots \text { alt }_{i} \|_{p} \ldots \text { alt }_{n}\right)\right] \\
& \text { 8. } C\left[\ldots\left(c x_{1} \ldots x_{n} \rightarrow s\right) \|_{1 . p} \ldots\right] \quad \mapsto C\left[\ldots\left(c x_{1} \ldots x_{n} \rightarrow s \|_{p}\right) \ldots\right] \\
& \text { 9. } C\left[\left(c s_{1} \ldots s_{n}\right) \|_{i . p}\right] \quad \mapsto C\left[\left(c s_{1} \ldots s_{i} \|_{p} \ldots s_{n}\right)\right] \\
& \text { 10. } C\left[(\text { letrec Env in } s) \|_{p}\right] \quad \mapsto C\left[\left(\text { letrec Env in } s \|_{p}\right)\right] \\
& \text { 11. } C_{1}\left[\left(\text { letrec } x=s, \text { Env in } C_{2}\left[x \|_{p}\right]\right)\right] \mapsto C_{1}\left[\left(\text { letrec } x=s \|_{p} \text {, Env in } C_{2}[x]\right)\right] \\
& \text { 12. } C_{1}\left[\operatorname{letrec} x=s, y=C_{2}\left[x \|_{p}\right], \quad \mapsto \quad C_{1}\left[\operatorname{letrec} x=s \|_{p}, y=C_{2}[x]\right. \text {, }\right. \\
& \text { 12. Env in } t] \quad \text { Env in } t] \\
& \text { 13. } C_{1}\left[\left(\text { letrec } x=C_{2}\left[x \|_{p}\right], \text { Env in } s\right)\right] \mapsto C_{1}\left[\left(\text { letrec } x=C_{2}[x] \|_{p}, \text { Env in } s\right)\right]
\end{aligned}
$$

If the position $\varepsilon$ hits the same (let-bound) variable twice, then the result is Bot. (This can only happen by a sequence of rules 11,12,13.)

Figure 9: Infinite tree construction from positions for fixed $s$

The set $\mathbb{C}_{\mathcal{I}}$ of infinite tree contexts includes any infinite tree where a subtree is replaced by a hole [·]. Reduction contexts on trees are defined as follows:

Definition 5.4. Call-by-name reduction contexts $\mathcal{R}_{\text {tree }}$ of $L_{\text {tree }}$ are defined as follows, where the grammar is interpreted inductively and $S \in \mathcal{E}_{\mathcal{I}}$ :

$$
R, R_{i} \in \mathcal{R}_{\text {tree }} \quad::=[\cdot]|(R S)|(\text { case } R \text { of alts }) \mid(\text { seq } R S)
$$

For an infinite tree, a reduction position $p$ is any position such that $p(S)$ is defined and there exists some $R \in \mathcal{R}_{\text {tree }}$ with $R\left[S^{\prime}\right]=S$ and $\left.R\right|_{p}=[\cdot]$

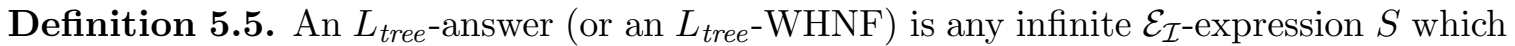
is an abstraction or constructor application, i.e. $\varepsilon(S)=\lambda x$ or $\varepsilon(S)=c$ for some constructor $c$. The reduction rules on infinite expressions are allowed in any context and are as follows:

(betaTr) $\quad\left(\left(\lambda x . S_{1}\right) S_{2}\right) \rightarrow S_{1}\left[S_{2} / x\right]$

(seqTr) (seq $\left.S_{1} S_{2}\right) \rightarrow S_{2}$ if $S_{1}$ is an $L_{\text {tree }}$-answer

$($ caseTr $) \quad\left(\operatorname{case}_{T}\left(c S_{1} \ldots S_{n}\right)\right.$ of $\left.\ldots\left(c x_{1} \ldots x_{n} \rightarrow S^{\prime}\right) \ldots\right) \rightarrow S^{\prime}\left[S_{1} / x_{1}, \ldots, S_{n} / x_{n}\right]$

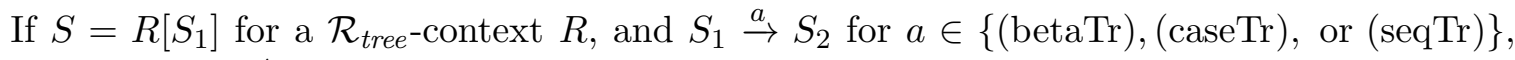
then we say $S \stackrel{\text { tree }}{\longrightarrow} S^{\prime}=R\left[S_{2}\right]$ is a normal order reduction (tree-reduction) on infinite trees. 
Here $S_{1}$ is the tree-redex of the tree-reduction. We also use the convergence predicate $\downarrow_{\text {tree }}$ for infinite trees defined as: $S \downarrow_{\text {tree }}$ iff $S \stackrel{\text { tree,* }}{\longrightarrow} S^{\prime}$ and $S^{\prime}$ is an $L_{\text {tree }}$-WHNF.

Note that $\stackrel{\text { tree,betaTr }}{\longrightarrow}$ and $\stackrel{\text { tree,caseTr }}{\longrightarrow}$ only reduce a single redex, but may modify infinitely many positions, since there may be infinitely many positions of a replaced variable $x$. E.g., a $($ tree,betaTr $)$ of $\operatorname{IT}((\lambda x .($ letrec $z=(z x)$ in $z)) r)=(\lambda x .((\ldots(\ldots x) x) x)) r$ $\rightarrow((\ldots(\ldots r) r) r)$ replaces the infinite number of occurrences of $x$ by $r$.

Concluding, the calculus $L_{\text {tree }}$ is defined by the tuple $\left(\mathcal{E}_{\mathcal{I}}, \mathbb{C}_{\mathcal{I}}, \stackrel{\text { tree }}{\longrightarrow}, \mathbb{A}_{\text {tree }}\right)$ where $\mathbb{A}_{\text {tree }}$ are the $L_{\text {tree-WHNFs. }}$-WHe

In the following we use a variant of infinite outside-in developments [Bar84, KKSdV97] as a reduction on trees that may reduce infinitely many redexes in one step. The motivation is that the infinite trees corresponding to finite expressions may require the reduction of infinitely many redexes of the trees for one $\stackrel{L R}{\longrightarrow}$ - or $\stackrel{L_{n a m e}}{\longrightarrow}$-reduction, respectively.

Definition 5.6. We define an infinite variant of Barendregt's 1-reduction: Let $S \in \mathcal{E}_{\mathcal{I}}$ be an infinite tree. Let $\dagger$ be a special label and $M$ be a set of (perhaps infinitely many) positions of $S$, which must be redexes w.r.t. the same reduction $a \in\{$ (betaTr), (caseTr), or (seqTr)\}. Now exactly all positions $m \in M$ of $S$ are labeled with $\dagger$. By $S \stackrel{I, M}{\longrightarrow} S^{\prime}$ we denote the (perhaps infinite) development top down, defined as follows:

- Let $S_{0}=S$ and $M_{0}=M$.

- Iteratively compute $M_{i+1}$ and $S_{i+1}$ from $M_{i}$ and $S_{i}$ for $i=0,1,2, \ldots$ as follows:

Let $d$ be the length of the shortest position in $M_{i}$, and $M_{i, d}$ be the finite set of positions that are the shortest ones in $M_{i}$.

For every $p \in M_{i, d}$ construct an infinite tree $T_{p}$ from $\left.S_{i}\right|_{p}$ by iterating the following reduction until the root of $\left.S_{i}\right|_{p}$ is not labeled: remove the label from the top of $\left.S_{i}\right|_{p}$, then perform a labeled reduction inheriting all the labels. If this iteration does not terminate, because the root of $\left.S_{i}\right|_{p}$ gets labeled in every step, then the result is $T_{p}:=\operatorname{Bot}$ (unlabeled), otherwise a result $T_{p}$ is computed after finitely many reductions.

Now construct $S_{i+1}$ by replacing every subtree at a position $p \in M_{i, d}$ in $S_{i}$ by $T_{p}$ : for the positions $p$ of $S_{i}$ that do not have a prefix that is in $M_{i, d}$, we set $p\left(S_{i+1}\right):=p\left(S_{i}\right)$ and for $p \in M_{i, d}$ we set $\left.S_{i}\right|_{p}:=T_{p}$.

Let $M_{i+1}$ be the set of positions in $S_{i+1}$ which carry a label $\dagger$. The length of the shortest position is now at least $d+1$. Then iterate again with $M_{i+1}, S_{i+1}$.

- $S^{\prime}$ is defined as the result after (perhaps infinitely many) construction steps $S_{1}, S_{2}, \ldots$

If the initial set $M$ does not contain a reduction position then we write $S \stackrel{I, M, \neg \text { tree }}{\longrightarrow} S^{\prime}$. We write $S \stackrel{I, \neg \text { tree }}{\longrightarrow} S^{\prime}\left(S \stackrel{I}{\rightarrow} S^{\prime}\right.$, resp.) if there exists a set $M$ such that $S \stackrel{I, M, \neg \text { tree }}{\longrightarrow} S^{\prime}$ $\left(S \stackrel{I, M}{\longrightarrow} S^{\prime}\right.$, resp.).

Example 5.7. We give two examples of standard reduction and $\stackrel{I, M}{\longrightarrow}$-reductions.

An $\stackrel{L R}{\longrightarrow}$-reduction on expressions corresponds to an $\stackrel{I, M}{\longrightarrow}$-reduction on infinite trees and perhaps corresponds to an infinite sequence of infinite tree-reductions. Consider letrec $y=$ $(\lambda x . y) a$ in $y$. The $(L R$, lbeta)-reduction with a subsequent $(L R$, llet) reduction results in letrec $y=y, x=a$ in $y$. The corresponding infinite tree of letrec $y=(\lambda x . y) a$ in $y$ is $S=\left(\left(\lambda x_{1} \cdot\left(\left(\lambda x_{2} \cdot\left(\left(\lambda x_{3} \cdot(\ldots a)\right) a\right)\right) a\right)\right) a\right)$. The (tree, betaTr)-reduction-sequence is infinite. let $M$ be the infinite set of positions of all the applications in $S$, i.e. $M=\{\varepsilon, 1.1,1.1 .1 .1, \ldots\}$. Then in the (infinite) development described in Def. 5.6 all intermediate trees have a label 
at the top, and thus we have $S \stackrel{I, M}{\longrightarrow}$ Bot. For a set $M$ without $\varepsilon$, the result will be a value tree.

For the expression letrec $y=($ seq True (seq $y$ False) $)$ in $y$ the $\stackrel{L R}{\longrightarrow}$-reduction results in the expression letrec $y=($ seq $y$ False) in $y$ which diverges. The corresponding infinite tree is (seq True (seq ((seq True (seq (...) False)) False))), which has an infinite number of tree-reductions, at an infinite number of deeper and deeper positions. Let $M=\{\varepsilon, 1.2,1.2 .1 .2, \ldots\}$ be the set consisting of all those positions. Then $S \stackrel{I, M}{\longrightarrow}$ (seq (seq (seq... False) False) False).

There may be $S, S^{\prime}$ such that $S \stackrel{I, M}{\longrightarrow} S^{\prime}$ as well as $S \stackrel{I, M^{\prime}}{\longrightarrow} S^{\prime}$ for some sets $M, M^{\prime}$ where $M$ contains a reduction position, but $M^{\prime}$ does not contain a reduction position. For example $S=\left(\lambda x_{1} \cdot x_{1}\right)\left(\left(\lambda x_{2} \cdot x_{2}\right)\left(\left(\lambda x_{3} \cdot x_{3}\right) \ldots\right)\right)$, where a single (betaTr)-reduction at the top reproduces $S$, as well as a single (betaTr)-reduction of the argument.

5.2. Standardization of Tree Reduction. Before considering the concrete calculi $L_{L R}$ and $L_{n a m e}$ and their correspondence to the calculus with infinite trees, we show that for an arbitrary reduction sequence on infinite trees resulting in an answer we can construct a tree-reduction sequence that results in an $L_{\text {tree }}$-WHNF.

Lemma 5.8. Let $T$ be an infinite expression. If $T \stackrel{I, M, \neg \text { tree }}{\longrightarrow} T^{\prime}$ for some $M$, where $T^{\prime}$ is an answer, then $T$ is also an answer.

Proof. This follows since an answer cannot be generated by $\stackrel{I, M, \neg \text { tree }}{\longrightarrow}$-reductions, since neither abstractions nor constructor expressions can be generated at the top position.

Lemma 5.9. Any overlapping between $a \stackrel{\text { tree }}{\longrightarrow}$-reduction and $a \stackrel{I, M}{\longrightarrow}$-reduction can be closed as follows. The trivial case that both given reductions are identical is omitted.

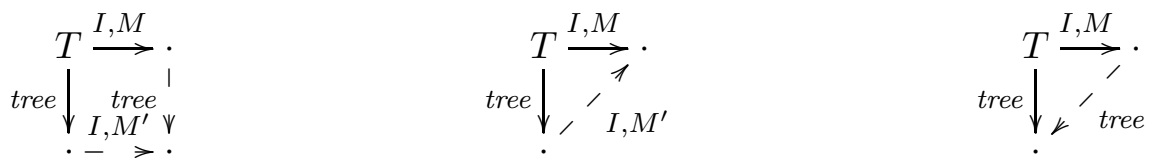

Proof. This follows by checking the overlaps of $\stackrel{I}{\rightarrow}$ with tree-reductions. The third diagram applies if the positions of $M$ are removed by the tree-reduction. The second diagram applies if the tree-redex is included in $M$ and the first diagram is applicable in all other cases.

Lemma 5.10. Let $T$ be an infinite tree such that there is a tree-reduction sequence of length $n$ to a WHNF $T^{\prime}$, and let $S$ be an infinite tree with $T \stackrel{I, M}{\longrightarrow} S$. Then $S$ has a tree-reduction sequence of length $\leq n$ to a WHNF $T^{\prime \prime}$.

Proof. This follows from Lemma 5.9 by induction on $n$.

Lemma 5.11. Consider two reductions $\stackrel{I, M_{1}}{\longrightarrow}$ and $\stackrel{I, M_{2}}{\longrightarrow}$ of the same type (betaTr), (caseTr) or (seqTr). For all trees $T, T_{1}, T_{2}:$ if $T \stackrel{I, M_{1}}{\longrightarrow} T_{1}$, and $T \stackrel{I, M_{2}}{\longrightarrow} T_{2}$, and $M_{2} \subseteq M_{1}$, then there is a set $M_{3}$ of positions, such that $T_{2} \stackrel{I, M_{3}}{\longrightarrow} T_{1}$.

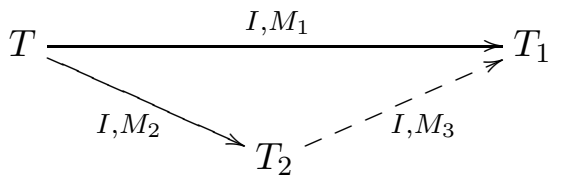


Proof. The argument is that the set $M_{3}$ is computed by labeling the positions in $T$ using $M_{1}$, and then by performing the infinite development using the set of redexes $M_{2}$, where we assume that the $M_{1}$-labels are inherited. The set of positions of marked redexes in $T_{2}$ that remain and are not reduced by $T_{1} \stackrel{I, M_{2}}{\longrightarrow} T_{2}$ is exactly the set $M_{3}$.

Consider a reduction $T \stackrel{I, M}{\longrightarrow} T^{\prime}$ of type (betaTr), (caseTr) or (seqTr). This reduction may include a redex of a normal order tree-reduction. Then the reduction can be split into $T \stackrel{\text { tree }}{\longrightarrow} T_{1} \stackrel{I}{\rightarrow} T^{\prime}$, and splitting of the reduction can be iterated as long as the remaining $T_{1} \stackrel{I}{\rightarrow} T^{\prime}$ has a tree-redex. It may happen that this process does not terminate.

We consider this non-terminating case, i.e. let $T_{0} \stackrel{I, M}{\longrightarrow} T^{\prime}$ and we can assume that there exist infinitely many $T_{1}, T_{2}, \ldots$ and $M_{1}, M_{2}, \ldots$, such that for any $k: T_{0} \stackrel{\text { tree, },}{\longrightarrow} T_{k}$ and $T_{k} \stackrel{I, M_{k}}{\longrightarrow} T^{\prime}$. By induction we can show for every $k \geq 1: T_{k-1}=R_{k-1}\left[S_{k-1}\right] \rightarrow R_{k-1}\left[S_{k}\right]=$ $T_{k}$ for a reduction context $R_{k}$ and where $S_{k-1}$ is the redex and $S_{k}$ is the contractum of $T_{k-1} \rightarrow T_{k}$ and the normal order tree-redex of $M_{k}$ labels a subterm of $S_{k}$. This holds, since the infinite development for $T \stackrel{I, M}{\longrightarrow} T^{\prime}$ is performed top down.

This implies that the infinite tree-reduction goes deeper and deeper along one path of the tree, or at some point all remaining tree-reductions are performed at the same position.

Lemma 5.12. Let $T \stackrel{I, M}{\longrightarrow} T^{\prime}$ such that $T^{\prime} \downarrow_{\text {tree }}$ and $M$ labels the normal order redex of $T$. Then there exists $T^{\prime \prime}$ and $M^{\prime}$ such that $T \stackrel{\text { tree,* }}{\longrightarrow} T^{\prime \prime} \stackrel{M^{\prime}, \neg \text { tree }}{\longrightarrow} T^{\prime}$.

Proof. Let $T=T_{0} \stackrel{\text { tree }, k}{\longrightarrow} T_{k}, T_{k} \stackrel{I, M_{k}}{\longrightarrow} T^{\prime}$ where $M_{k}$ labels a normal order redex.

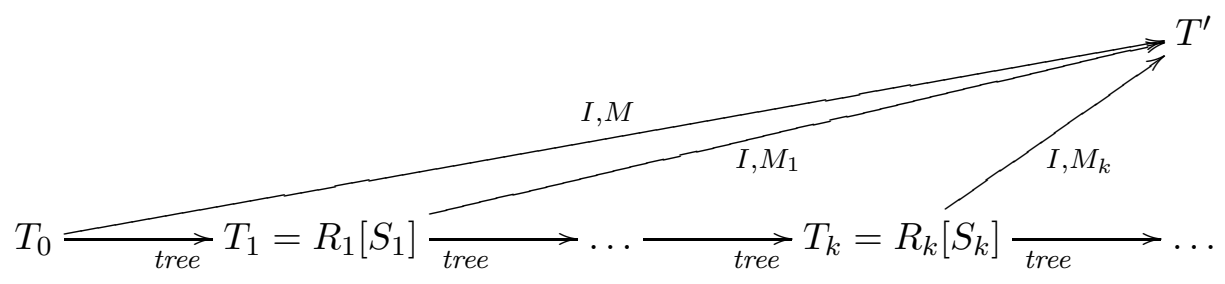

We have $T_{k}=R_{k}\left[S_{k}\right]$ where $R_{k}$ is a reduction context, and $M_{k}$ labels the hole of $R_{k}$, which is the normal order redex. The normal order reduction is $T_{k}=R_{k}\left[S_{k}\right] \stackrel{\text { tree }}{\longrightarrow} R_{k}\left[S_{k}^{\prime}\right]=: T_{k+1}$. Let $p_{k}$ be the path of the hole of $R_{k}$, together with the constructors and symbols (case, seq, constructors and @) on the path. Also let $M_{k}=M_{k, 1} \cup M_{k, 2}$, (where $\bullet$ means disjoint union) where the labels of $M_{k, 1}$ are in $R_{k}$, and the labels $M_{k, 2}$ are in $S_{k}$. Lemma 5.11, the structure of the expressions and the properties of the infinite top down developments show that the normal order redex can only stay or descend, i.e. $h>k$ implies that $p_{k}$ is a prefix of $p_{h}$.

Also, we have $R_{k}\left[S_{k}\right] \stackrel{I, M_{k}}{\longrightarrow} R_{k}^{\prime}\left[S^{\prime}\right]$, where $R_{k}[\cdot] \stackrel{M_{k, 1}}{\longrightarrow} R_{k}^{\prime}[\cdot]$, and $S_{k} \stackrel{I}{\rightarrow} S^{\prime}$.

There are three cases:

- The normal order reduction of $T_{0}$ halts, i.e., there is a maximal $k$. Then obviously $T \stackrel{\text { tree,*}}{\longrightarrow} T_{k} \stackrel{M_{k}, \neg \text { tree }}{\longrightarrow} T^{\prime}$.

- There is some $k$, such that $R_{k}=R_{h}$ for all $h \geq k$. In this case, $T^{\prime}=R_{k}^{\prime}\left[s^{\prime}\right]$. The infinite development $T_{0} \stackrel{I, M}{\longrightarrow} T^{\prime}$ will reduce infinitely often at the position of the hole, hence it will plug a Bot at position $p_{k}$ of $T^{\prime}$, and so $T^{\prime}=R_{k}^{\prime}[$ Bot $]$. But then $T^{\prime}$ cannot converge, and so this case is not possible. 
- The positions $p_{k}$ of the reduction contexts $R_{k}$ will grow indefinitely. Then there is an infinite path (together with the constructs and symbols) $p$ such that $p_{k}$ is a prefix of $p$ for every $k$. Moreover, $p$ is a position of $T^{\prime}$. The sets $M_{k, 1}$ are an infinite ascending set w.r.t. $\subseteq$, hence there is a limit tree $T_{\infty}$ with $T \stackrel{\text { tree }, \infty}{\longrightarrow} T_{\infty}$, which is exactly the limit of the contexts $R_{k}$ for $k \rightarrow \infty$. There is a reduction $T_{\infty} \stackrel{I, M^{\prime}}{\longrightarrow} T^{\prime}$ which is exactly $M^{\prime}=\bigcup_{k} M_{k, 1}$. Hence $T^{\prime}$ has the path $p$, and we see that the tree $T^{\prime}$ cannot have a normal order redex, since the search for such a redex goes along $p$ and thus does not terminate. This is a contradiction, and hence this case is not possible.

Lemma 5.13. Let $T \stackrel{I, M, \neg \text { tree }}{\longrightarrow} T_{1} \stackrel{\text { tree }}{\longrightarrow} T^{\prime}$. Then the reduction can be commuted to $T \stackrel{\text { tree }}{\longrightarrow}$ $T_{3} \stackrel{I, M^{\prime}}{\longrightarrow} T^{\prime}$ for some $M^{\prime}$.

Proof. This follows since the $\stackrel{I, M, \neg \text { tree }}{\longrightarrow}$-reduction cannot generate a new normal order treeredex. Hence, the normal order redex of $T_{1}$ also exists in $T$. The set $M^{\prime}$ can be found by labeling $T$ with $M$, then performing the tree-reduction where all labels of $M$ are kept and inherited by the reduction, except for those positions which are removed by the reduction.

Lemma 5.14. Let $T \stackrel{I, \neg \text { tree }}{\longrightarrow} T^{\prime}$ and $T^{\prime} \downarrow_{\text {tree }}$. Then $T \downarrow_{\text {tree }}$.

Proof. We show by induction on $k$ that whenever $T \stackrel{I, \neg \text { tree }}{\longrightarrow} T^{\prime} \stackrel{\text { tree, } k}{\longrightarrow} T^{\prime \prime}$ where $T^{\prime \prime}$ is an $L_{\text {tree- }}$ WHNF, then $T \downarrow_{\text {tree }}$. The base case is $k=0$ and it holds by Lemma 5.8. For the induction step let $T \stackrel{I, \neg \text { tree }}{\longrightarrow} T^{\prime} \stackrel{\text { tree }}{\longrightarrow} T_{0} \stackrel{\text { tree }, k}{\longrightarrow} T^{\prime \prime}$. We apply Lemma 5.13 to $T \stackrel{I, \neg \text { tree }}{\longrightarrow} T^{\prime} \stackrel{\text { tree }}{\longrightarrow} T_{0}$ and thus have $T \stackrel{\text { tree }}{\longrightarrow} T_{1} \stackrel{I, M}{\longrightarrow} T_{0} \stackrel{\text { tree }, k}{\longrightarrow} T^{\prime \prime}$ for some $M$.

This situation can be depicted by the following diagram where the dashed reductions follow by Lemma 5.13.

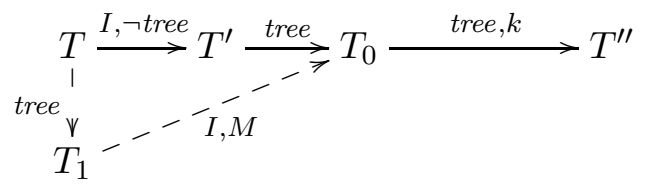

If $M$ does not contain a normal order redex, then the induction hypothesis shows that $T_{1} \downarrow_{\text {tree }}$ and thus also $T \downarrow_{\text {tree }}$. Now assume that $M$ contains a normal order redex. Then we apply Lemma 5.12 to $T_{1} \stackrel{I, M}{\longrightarrow} T_{0}$ (note that $T_{0} \downarrow_{\text {tree }}$ and hence the lemma is applicable). This shows that $T_{1} \stackrel{\text { tree,* }}{\longrightarrow} T_{0}^{\prime \prime} \stackrel{I, \neg \text { tree }}{\longrightarrow} T_{0}$ :

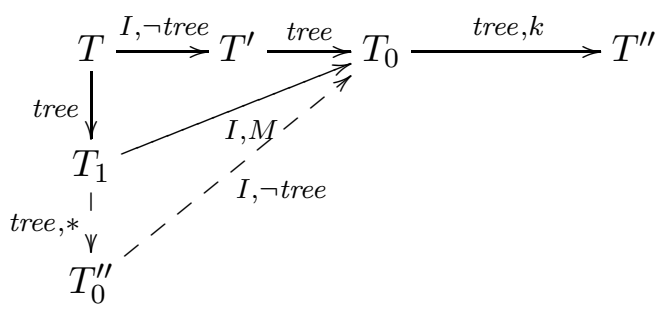

Now we can apply the induction hypothesis to $T_{0}^{\prime \prime} \stackrel{\neg \text { tree }}{\longrightarrow} T_{0} \stackrel{\text { tree,k}}{\longrightarrow} T^{\prime \prime}$ and have $T_{0}^{\prime \prime} \downarrow_{\text {tree }}$ which also shows $T \downarrow_{\text {tree }}$. 
Proposition 5.15 (Standardization). Let $T_{1}, \ldots, T_{k}$ be infinite trees such that $T_{k} \stackrel{I, M_{k-1}}{\longrightarrow}$ $T_{k-1} \stackrel{I, M_{k-2}}{\longrightarrow} T_{k-2} \ldots \stackrel{I, M_{1}}{\longrightarrow} T_{1}$, where $T_{1}$ is an $L_{\text {tree }}-W H N F$. Then $T_{k} \downarrow_{\text {tree }}$

Proof. We use induction on $k$. If $k=1$ then the claim obviously holds since $T_{k}=T_{1}$ is already an $L_{\text {tree }}$-WHNF. For the induction step assume that $T_{i} \stackrel{I, M_{i-1}}{\longrightarrow} T_{i-1} \ldots \stackrel{I, M_{1}}{\longrightarrow} T_{1}$ and $T_{i} \downarrow_{\text {tree }}$. Let $T_{i+1} \stackrel{I, M_{i}}{\longrightarrow} T_{i}$. If $M_{i}$ contains a normal order redex, then we apply Lemma 5.12 and have the following situation

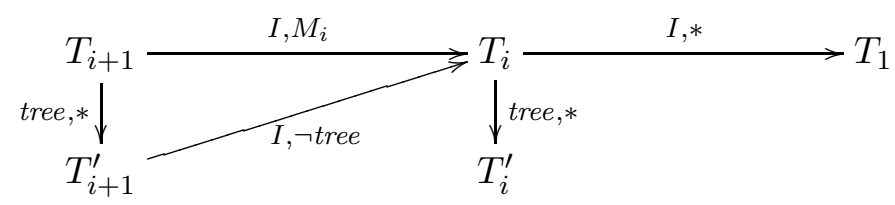

where $T_{i}^{\prime}$ is an $L_{\text {tree }}$ WHNF. We apply Lemma 5.14 to $T_{i+1}^{\prime} \stackrel{I, \neg \text { tree }}{\longrightarrow} T_{i} \stackrel{\text { tree,* }}{\longrightarrow} T_{i}^{\prime}$ which shows that $T_{i+1}^{\prime} \downarrow_{\text {tree }}$ and thus also $T_{i+1} \downarrow_{\text {tree }}$.

If $M_{i}$ contains no normal order redex, we have

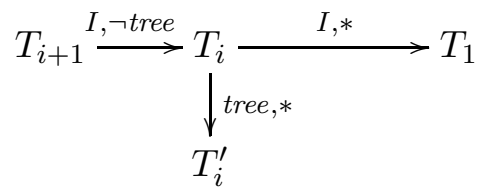

where $T_{i}^{\prime}$ is an $L_{\text {tree }}$-WHNF. We apply Lemma 5.14 to $T_{i+1} \stackrel{I, \neg \text { tree }}{\longrightarrow} T_{i} \stackrel{\text { tree,* }}{\longrightarrow} T_{i}^{\prime}$ and have $T_{i+1} \downarrow_{\text {tree }}$.

5.3. Equivalence of Tree-Convergence and $L_{L R}$-Convergence. In this section we will show that $L_{L R}$-convergence for finite expressions $s \in \mathbb{E}_{\mathcal{L}}$ coincides with convergence for the corresponding infinite tree $I T(s)$.

Lemma 5.16. Let $s_{1}, s_{2} \in \mathbb{E}_{\mathcal{L}}$ be finite expressions and $s_{1} \rightarrow s_{2}$ by a rule (cp), or (1ll). Then $\operatorname{IT}\left(s_{1}\right)=\operatorname{IT}\left(s_{2}\right)$.

Lemma 5.17. Let $s$ be a finite expression. If $s$ is an $L_{L R}-W H N F$ then $I T(s)$ is an answer. If $\operatorname{IT}(s)$ is an answer, then $s \downarrow_{L R}$.

Proof. If $s$ is an $L_{L R}$-WHNF, then obviously, $\operatorname{IT}(s)$ is a answer. If $\operatorname{IT}(s)$ is an answer, then the label computation of the infinite tree for the empty position using s, i.e. $s \|_{\varepsilon}$, must be $\lambda x$ or $c$ for some constructor. If we consider all the cases where the label computation for $s \|_{\varepsilon}$ ends with such a label, we see that $s$ must be of the form $N L[v]$, where $v$ is an $L_{L R}$-answer and the contexts $N L$ are constructed according to the grammar:

$$
\begin{aligned}
N L::= & {[\cdot] \mid \text { letrec Env in } N L } \\
& \mid \text { letrec } x_{1}=N L[\cdot],\left\{x_{i}=N L\left[x_{i-1}\right]\right\}_{i=2}^{n}, \text { Env in } N L\left[x_{n}\right]
\end{aligned}
$$

We show by induction that every expression $N L[v]$, where $v$ is a value, can be reduced by normal order (cp)- and (llet)-reductions to a WHNF in $L_{L R}$. We use the following 
induction measure $\mu$ on $N L[v]$ :

$$
\begin{aligned}
\mu(v) & :=0 \\
\mu(\text { letrec Env in } N L[v]) & :=1+\mu(N L[v]) \\
\mu\left(\text { letrec } x_{1}=N L_{1}[v],\left\{x_{i}=N L_{i}\left[x_{i-1}\right]\right\}_{i=2}^{n}, \text { Env in } N L_{n+1}\left[x_{n}\right]\right) & := \\
\mu\left(N L_{1}[v]\right)+\mu\left(\operatorname{letrec} x_{2}=N L_{2}[v],\left\{x_{i}=N L_{i}\left[x_{i-1}\right]\right\}_{i=3}^{n}, E n v\right. & \text { in } \left.N L_{n+1}\left[x_{n}\right]\right)
\end{aligned}
$$

The base case obviously holds, since $v$ is already an $L_{L R}$-WHNF. For the induction step assume that $N L\left[v^{\prime}\right] \stackrel{L R, \text { cp Vllet, } *}{\longrightarrow} t$, where $t$ is an $L_{L R}$-WHNF for every $N L\left[v^{\prime}\right]$ with $\mu\left(N L\left[v^{\prime}\right]\right)<$ $k$. Let $N L$, and $v$ be fixed, such that $\mu(N L[v])=k \geq 1$. There are two cases:

- $N L[v]=$ letrec Env in $N L^{\prime}[v]$. If $N L^{\prime}$ is the empty context, then $N L[v]$ is an $L_{L R}$-WHNF. Otherwise $N L^{\prime}[v]$ is a letrec-expression. Thus we can apply an (LR, (llet-in))-reduction to $N L[v]$, where the measure $\mu$ is decreased by one. The induction hypothesis shows the claim.

- $N L[v]=$ letrec $x_{1}=N L_{1}[v],\left\{x_{i}=N L_{i}\left[x_{i-1}\right]\right\}_{i=2}^{n}$, Env in $N L_{n+1}\left[x_{n}\right]$. If $N L_{n+1}\left[x_{n}\right]$ is a letrec-expression, then we can apply an ( $L R$, llet-in)-reduction to $N L[v]$ and the measure $\mu$ is decreased by 1 . If $N L_{n+1}$ is the empty context, and there is some $i$ such that $N L_{i}$ is not the empty context, then we can choose the largest number $i$ and apply an ( $L R$, llet-e)-reduction to $N L[v]$. Then the measure $\mu$ is strictly decreased and we can use the induction hypothesis. If all the contexts $N L_{i}$ for $i=1, \ldots, n+1$ are empty contexts, then either $N L[v]$ is an $L_{L R}$-WHNF (if $v$ is a constructor application) or we can apply an $(L R, \mathrm{cp})$ reduction to obtain an $L_{L R}$-WHNF.

Lemma 5.18. Let $s \in \mathbb{E}_{\mathcal{L}}$ such that $s \stackrel{\text { LR,a }}{\longrightarrow} t$. If the reduction $a$ is (cp) or (lll) then $I T(s)=I T(t)$. If the reduction $a$ is (lbeta), (case-c), (case-in), (case-e) or (seq-c), (seq-in),(seq-c) then $I T(s) \stackrel{I, M, a^{\prime}}{\longrightarrow} I T(t)$ for some $M$, where $a^{\prime}$ is (betaTr), (caseTr), or (seqTr), respectively, and the set $M$ contains normal order redexes.

Proof. Only the latter needs a justification. Therefore, we label every redex in $\operatorname{IT}(s)$ that is derived from the redex $s \stackrel{L R}{\longrightarrow} t$ by $I T($.$) . This results in the set M$ for $I T(s)$. There will be at least one position in $M$ that is a normal order redex of $\operatorname{IT}(s)$.

Proposition 5.19. Let $s \in \mathbb{E}_{\mathcal{L}}$ such that $s \downarrow_{L R}$. Then $\operatorname{IT}(s) \downarrow_{\text {tree }}$.

Proof. We assume that $s \stackrel{L R, *}{\longrightarrow} t$, where $t$ is a WHNF. Using Lemma 5.18, we see that there is a finite sequence of reductions $I T(s) \stackrel{I, *}{\longrightarrow} I T(t)$. Lemma 5.17 shows that $I T(r)$ is an $L_{\text {tree }}$-WHNF. Now Proposition 5.15 shows that $I T(s) \downarrow_{\text {tree }}$.

We now consider the other direction and show that for every expression $s$ : if $\operatorname{IT}(s)$ converges, then $s$ converges, too.

Lemma 5.20. Let $R$ be some reduction context, s.t. $I T(s)=R[T] \stackrel{\text { tree, } a^{\prime}}{\longrightarrow} R\left[T^{\prime}\right]$. Then for $\left(a^{\prime}, a\right) \in\left\{(\right.$ betaTr, lbeta), (caseTr, case), (seqTr, seq) $\}$ there exist expressions $s_{1}, s_{2}, s_{3}$ and an infinite tree $T^{\prime}$ with $s \stackrel{L R, 111, *}{\longrightarrow} s_{1} \stackrel{L R, \mathrm{cp}, 0 \vee 1}{\longrightarrow} s_{2} \stackrel{L R, a}{\longrightarrow} s_{3}$ with $R\left[T^{\prime}\right] \stackrel{I, M}{\longrightarrow} \operatorname{IT}\left(s_{3}\right)$. 


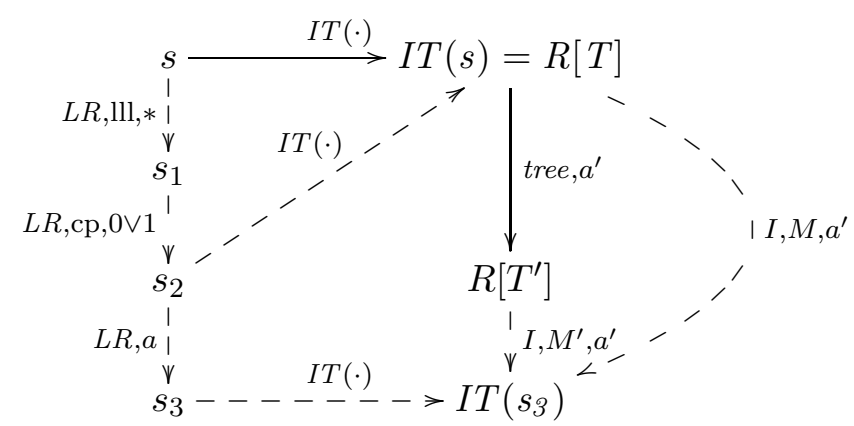

Proof. Let $p$ be the position of the hole of $R$. We follow the label computation to $T$ along $p$ inside $s$ and show that the redex corresponding to $T$ can be found in $s$ after some (lll) and (cp) reductions. For applications, seq-expressions, and case-expressions there is a one-toone correspondence. If the label computation shifts a position into a "deep" letrec, i.e. $C\left[(\right.$ letrec Env in $\left.s) \|_{p}\right] \mapsto C\left[\left(\right.\right.$ letrec Env in $\left.\left.s \|_{p}\right)\right]$ where $C$ is non-empty, then a sequence of normal order (lll)-reduction moves the environment Env to the top of the expression, where perhaps it is joined with a top-level environment of $C$. Let $s \stackrel{L R, 111, *}{\longrightarrow} s^{\prime}$. Lemma 5.16 shows that $I T\left(s^{\prime}\right)=I T(s)$ and the label computation along $p$ for $s^{\prime}$ requires fewer steps than the computation for $s$. Hence this construction can be iterated and terminates. This yields a reduction sequence $s \stackrel{L R, l l l, *}{\longrightarrow} s_{1}$ such that the label computation along $p$ for $s_{1}$ does not shift the label into deep letrecs and where $I T(s)=I T\left(s_{1}\right)$ (see Lemma 5.16). Now there are two cases: Either the redex corresponding to $T$ is also a normal order redex of $s_{1}$, or $s_{1}$ is of the form letrec $x_{1}=\lambda x \cdot s^{\prime}, x_{2}=x_{1}, \ldots, x_{m}=x_{m-1}, \ldots R^{\prime}\left[x_{m}\right] \ldots$ For the latter case an $(L R, \mathrm{cp})$ reduction is necessary before the corresponding reduction rule can be applied. Again Lemma 5.16 assures that the infinite tree remains unchanged. After applying the corresponding reduction rule, i.e. $s_{2} \stackrel{L R, a}{\longrightarrow} s_{3}$, the normal order reduction may have changed infinitely many positions of $I T\left(s_{3}\right)$, while $R[T] \stackrel{\text { tree }, a^{\prime}}{\longrightarrow} R\left[T^{\prime}\right]$ does not change all these positions, but nevertheless Lemma 5.18 shows that there is a reduction $R[T] \stackrel{I, M, a^{\prime}}{\longrightarrow} I T\left(s_{3}\right)$, and Lemma 5.11 shows that also $R\left[T^{\prime}\right] \stackrel{I, M^{\prime}, a^{\prime}}{\longrightarrow} I T\left(s_{3}\right)$ for some $M^{\prime}$.

Example 5.21. An example for the proof of the last lemma is the expression $s$ defined as $s:=$ letrec $x=(\lambda y . y) x$ in $x$. Then $I T(s)=(\lambda y \cdot y)((\lambda y \cdot y)((\lambda y \cdot y) \ldots))$. The tree-reduction for $I T(s)$ is $I T(s) \stackrel{\text { tree,betaTr }}{\longrightarrow} I T(s)$. On the other hand the normal order reduction of $L_{L R}$ reduces to $s^{\prime}:=$ letrec $x=($ letrec $y=x$ in $y)$ in $x$ and $I T\left(s^{\prime}\right)=$ Bot. To join the reductions we perform an $\stackrel{I, M}{\longrightarrow}$-reduction for $I T(s)$ where all redexes are labeled in $M$, which also results in Bot.

Proposition 5.22. Let $s$ be an expression such that $I T(s) \downarrow_{\text {tree }}$. Then $s \downarrow_{L R}$.

Proof. The precondition $I T(s) \downarrow_{\text {tree }}$ implies that there is a tree-reduction sequence of $\operatorname{IT}(s)$

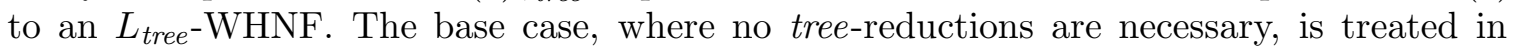
Lemma 5.17. In the general case, let $T \stackrel{\text { tree, } a^{\prime}}{\longrightarrow} T^{\prime}$ be a tree-reduction. Lemma 5.20 shows that there are expressions $s^{\prime}, s^{\prime \prime}$ with $s \stackrel{L R, 111, *}{\longrightarrow} \stackrel{L R, \mathrm{cp}, 0 \vee 1}{\longrightarrow} s^{\prime} \stackrel{L R, a}{\longrightarrow} s^{\prime \prime}$, and $T^{\prime} \stackrel{I, M}{\longrightarrow} \operatorname{IT}\left(s^{\prime \prime}\right)$. Lemma 5.10 shows that $I T\left(s^{\prime \prime}\right)$ has a normal order tree-reduction to a WHNF where the number of tree-reductions is strictly smaller than the number of tree-reductions of $T$ to a 
WHNF. Thus we can use induction on this length and obtain a normal order $L R$-reduction of $s$ to a WHNF.

Propositions 5.19 and 5.22 imply the theorem

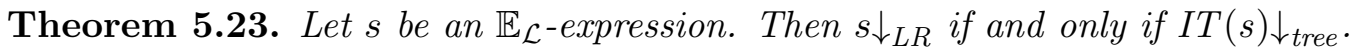

5.4. Equivalence of Infinite Tree Convergence and $L_{n a m e}$-convergence. It is easy to observe that several reductions of $L_{\text {name }}$ do not change the infinite trees w.r.t. the translation $\operatorname{IT}(\cdot)$ :

Lemma 5.24. Let $s_{1}, s_{2} \in \mathbb{E}_{\mathcal{L}}$. Then $s_{1} \stackrel{\text { name, } a}{\longrightarrow} s_{2}$ for $a \in\{$ gcp, lapp, lcase, lseq $\}$ implies $\operatorname{IT}\left(s_{1}\right)=I T\left(s_{2}\right)$.

Lemma 5.25. For $\left(a, a^{\prime}\right) \in\{($ beta, betaTr), (case, caseTr), (seq, seqTr) $\}$ it holds:

If $s_{1} \stackrel{\text { name }, a}{\longrightarrow} s_{2}$ for $s_{i} \in \mathbb{E}_{\mathcal{L}}$, then $\operatorname{IT}\left(s_{1}\right) \stackrel{\text { tree }, a^{\prime}}{\longrightarrow} \operatorname{IT}\left(s_{2}\right)$.

Proof. Let $s_{1}:=R_{\text {name }}\left[s_{1}^{\prime}\right] \stackrel{\text { name,a }}{\longrightarrow} R_{\text {name }}\left[s_{2}^{\prime}\right]=s_{2}$ where $s_{1}^{\prime}$ is the redex of the $\stackrel{\text { name }}{\longrightarrow \text { - }}$ reduction and $R_{\text {name }}$ is an $L_{\text {name }}$-reduction context. First one can observe that the redex $s_{1}^{\prime}$ is mapped by $I T$ to a unique tree position within a tree reduction context in $\operatorname{IT}\left(s_{1}\right)$.

We only consider the (beta)-reduction, since for a (case)- or a (seq)-reduction the reasoning is completely analogous. So let us assume that $s_{1}^{\prime}=\left(\left(\lambda x . s_{1}^{\prime \prime}\right) s_{2}^{\prime \prime}\right)$. Then $I T$ transforms $s_{1}^{\prime}$ into a subtree $\sigma\left(\left(\lambda x . I T\left(s_{1}^{\prime \prime}\right)\right) I T\left(s_{2}^{\prime \prime}\right)\right)$ where $\sigma$ is a substitution replacing variables by infinite trees. The tree reduction replaces $\sigma\left(\left(\lambda x . \operatorname{IT}\left(s_{1}^{\prime \prime}\right)\right) \operatorname{IT}\left(s_{2}^{\prime \prime}\right)\right)$ by $\sigma\left(\operatorname{IT}\left(s_{1}^{\prime \prime}\right)\right)\left[\sigma\left(\operatorname{IT}\left(s_{2}^{\prime \prime}\right)\right) / x\right]$, hence the lemma holds.

Proposition 5.26. Let $s \in \mathbb{E}_{\mathcal{L}}$ be an expression with $s \downarrow_{\text {name }}$. Then $\operatorname{IT}(s) \downarrow_{\text {tree }}$.

Proof. This follows by induction on the length of a normal order reduction of $s$. The base case holds since $I T(L[v])$, where $v$ is an $L_{n a m e}$-answer is always an $L_{\text {tree-answer. For }}$ the induction step we consider the first reduction of $s$, say $s \stackrel{\text { name }}{\longrightarrow} s^{\prime}$. The induction hypothesis shows $I T\left(s^{\prime}\right) \downarrow_{\text {tree }}$. If the reduction $s \stackrel{\text { name }}{\longrightarrow} s^{\prime}$ is (name,gcp), (name,lapp), (name,lcase), or (name,lseq), then Lemma 5.24 implies $I T(s) \downarrow_{\text {tree }}$. If $s \stackrel{\text { name,a }}{\longrightarrow} s^{\prime}$ for $a \in\{($ beta $)$, (case), (seq) $\}$, then Lemma 5.25 shows $\operatorname{IT}(s) \stackrel{\text { tree }}{\longrightarrow} \operatorname{IT}\left(s^{\prime}\right)$ and thus $\operatorname{IT}(s) \downarrow_{\text {tree }}$.

Now we show the other direction:

Lemma 5.27. Let $s \in \mathbb{E}_{\mathcal{L}}$ such that $I T(s)=\mathcal{R}[T]$, where $\mathcal{R}$ is a tree reduction context and $T$ is a value or a redex. Then there are expressions $s^{\prime}, s^{\prime \prime}$ such that $s \stackrel{\text { name,lapp } \vee l c a s e V l s e q \vee g c p, *}{\longrightarrow}$ $s^{\prime}, I T\left(s^{\prime}\right)=I T(s), s^{\prime}=R\left[s^{\prime \prime}\right], I T\left(L\left[s^{\prime \prime}\right]\right)=T$, where $R=L[A[\cdot]]$ is a reduction context for some $\mathcal{L}$-context $L$ and some $\mathcal{A}$-context $A$, $s^{\prime \prime}$ may be an abstraction, a constructor application, or a beta-, case- or seq-redex iff $T$ is an abstraction, a constructor application, or a betaTr-, caseTr- or seqTr-redex, respectively, and the position $p$ of the hole in $\mathcal{R}$ is also the position of the hole in $A[\cdot]$.

Proof. The tree $T$ may be an abstraction, a constructor application, an application, or a betaTr-, caseTr- or seqTr-redex in $R[T]$. Let $p$ be the position of the hole of $\mathcal{R}$. We will show by induction on the label-computation for $p$ in $s$ that there is a reduction $s \stackrel{\text { name,lapp } \vee \mathrm{lcase} \vee \mathrm{lseq} \vee \mathrm{gcp}, *}{\longrightarrow} s^{\prime}$, where $s^{\prime}$ is as claimed in the lemma. 
We consider the label-computation for $p$ to explain the induction measure, where we use the numbers of the rules given in Fig. 9. Let $q$ be such that the label computation for $p$ is of the form $(10)^{*} \cdot q$ and $q$ does not start with (10). The measure for induction is a tuple $(a, b)$, where $a$ is the length of $q$, and $b \geq 0$ is the maximal number with $q=(2 \vee 4 \vee 6)^{b} \cdot q^{\prime}$. The base case is $(a, a)$ : Then the label computation is of the form $(2 \vee 4 \vee 6)^{*}$ and indicates that $s$ is of the form $L\left[A\left[s^{\prime \prime}\right]\right]$ and satisfies the claim of the lemma. For the induction step we have to check several cases:

(1) The label computation starts with $(10)^{*}(2 \vee 4 \vee 6)^{+}(10)$. Then a normal-order (lapp), (lcase), or (lseq) can be applied to $s$ resulting in $s_{1}$. The label-computation for $p$ w.r.t. $s_{1}$ is of the same length, and only applications of (10) and $(2 \vee 4 \vee 6)$ are interchanged, hence the second component of the measure is strictly decreased.

(2) The label computation starts with $(10)^{*}(2 \vee 4 \vee 6)^{*}(11)$. Then a normal-order (gcp) can be applied to $s$ resulting in $s_{1}$. The length $q$ is strictly decreased by 1 , and perhaps one (12)-step is changed into a (11)-step. Hence the measure is strictly decreased.

In every case the claim on the structure of the contexts and $s^{\prime}$ can easily be verified.

Lemma 5.28. Let $s$ be an expression with $I T(s) \stackrel{\text { tree }}{\longrightarrow} T$. Then there is some $s^{\prime}$ with $s \stackrel{\text { name,* }}{\longrightarrow} s^{\prime}$ and $I T\left(s^{\prime}\right)=T$.

Proof. If $I T(s) \stackrel{\text { tree }}{\longrightarrow} T$, then $I T(s)=\mathcal{R}[S]$ where $\mathcal{R}$ is a reduction context, $S$ a tree-redex with $S \stackrel{\text { tree }}{\longrightarrow} S^{\prime}$ and $T=\mathcal{R}\left[S^{\prime}\right]$. Let $p$ be the position of the hole of $\mathcal{R}$ in $\operatorname{IT}(s)$. We apply Lemma 5.27, which implies that there is a reduction $s \stackrel{\text { name,* }}{\longrightarrow} s^{\prime}$, such that $\operatorname{IT}(s)=\operatorname{IT}\left(s^{\prime}\right)$ and $s^{\prime}=R\left[s^{\prime \prime}\right]$ where $R=L[A[\cdot]]$ is a reduction context and $I T\left(L\left[s^{\prime \prime}\right]\right)$ is a beta-, case-, or seq-redex. It is obvious that $s^{\prime}=L\left[A\left[s^{\prime \prime}\right]\right] \stackrel{\text { name,a }}{\longrightarrow} t$. Now one can verify that $I T(t)=T$ must hold.

Proposition 5.29. Let $s$ be an expression with $I T(s) \downarrow_{\text {tree }}$. Then $s \downarrow_{\text {name }}$.

Proof. We use induction on the length $k$ of a tree reduction $I T(s) \stackrel{\text { tree }, k}{\longrightarrow} T$, where $T$ is an $L_{\text {tree-answer. }}$ For the base case it is easy to verify that if $I T(s)$ is an $L_{\text {tree-answer, then }}$

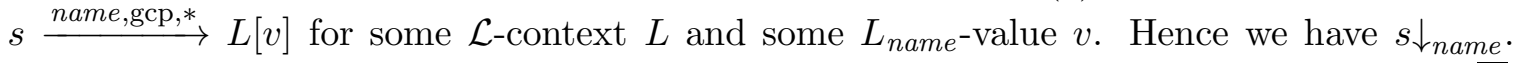
The induction step follows by repeated application of Lemma 5.28.

Corollary 5.30. For all $\mathbb{E}_{\mathcal{L}}$-expressions $s: s \downarrow_{\text {name }}$ if, and only if $\operatorname{IT}(s) \downarrow_{\text {tree }}$.

Theorem 5.31. $\leq_{\text {name }}=\leq_{L R}$.

Proof. In Corollary 5.30 we have shown that $L_{\text {name-convergence is equivalent to infinite }}$ tree convergence. In Theorem 5.23 we have shown that $L_{L R}$-convergence is equivalent to infinite tree convergence. Hence, $L_{n a m e}$-convergence and $L_{L R}$-convergence are equivalent, which further implies that both contextual preorders and also the contextual equivalences are identical.

Corollary 5.32. The translation $W$ is convergence equivalent and fully abstract.

Since $W$ is the identity on expressions, this implies:

Corollary 5.33. $W$ is an isomorphism according to Definition 2.7. 
A further consequence of our results is that the general copy rule (gcp) is a correct program transformation in $L_{L R}$. This is a novel result, since in previous work only special cases were proved correct.

Proposition 5.34. The program transformation (gcp) is correct in $L_{n a m e}$ and $L_{L R}$.

Proof. Correctness of (gcp) in $L_{\text {name }}$ holds, since for $s, t \in \mathbb{E}_{\mathcal{L}}$ with $s \stackrel{g c p}{\longrightarrow} t$ and for any context $C: I T(C[s])=I T(C[t])$. Hence Corollary $\left[5.30\right.$ implies that $C[s] \downarrow_{\text {name }} \Longleftrightarrow C[t] \downarrow_{\text {name }}$ and thus $s \sim_{\text {name }} t$. Theorem 5.31 finally also shows $s \sim_{L R} t$.

\section{The Translation $N: L_{n a m e} \rightarrow L_{l c c}$}

We use multi-fixpoint combinators as defined in Gol05] to translate letrec-expressions $\mathbb{E}_{\mathcal{L}}$ of the calculus $L_{\text {name }}$ into equivalent ones without a letrec. The translated expressions are $\mathbb{E}_{\lambda}$ and belong to the calculus $L_{l c c}$.

Definition 6.1. Given $n \geq 1$, a family of $n$ fixpoint combinators $\boldsymbol{Y}_{i}^{n}$ for $i=1, \ldots, n$ can be defined as follows:

$$
\begin{aligned}
& \boldsymbol{Y}_{i}^{n}:=\lambda f_{1}, \ldots, f_{n} \cdot\left(\quad\left(\lambda x_{1}, \ldots, x_{n} \cdot f_{i} \quad\left(x_{1} x_{1} \ldots x_{n}\right) \ldots\left(x_{n} x_{1} \ldots x_{n}\right)\right)\right. \\
& \left(\lambda x_{1}, \ldots, x_{n} \cdot f_{1} \quad\left(x_{1} x_{1} \ldots x_{n}\right) \ldots\left(x_{n} x_{1} \ldots x_{n}\right)\right) \\
& \text {... } \\
& \left.\left(\lambda x_{1}, \ldots, x_{n} \cdot f_{n} \quad\left(x_{1} x_{1} \ldots x_{n}\right) \ldots\left(x_{n} x_{1} \ldots x_{n}\right)\right)\right)
\end{aligned}
$$

The idea of the translation is to replace (letrec $x_{1}=s_{1}, \ldots, x_{n}=s_{n}$ in $t$ ) by $t\left[B_{1} / x_{1}, \ldots, B_{n} / x_{n}\right]$ where $B_{i}:=\boldsymbol{Y}_{i}^{n} F_{1} \ldots F_{n}$ and $F_{i}:=\lambda x_{1}, \ldots, x_{n} . s_{i}$.

In this way the fixpoint combinators implement the generalized fixpoint property: $\boldsymbol{Y}_{i}^{n} F_{1} \ldots F_{n} \sim F_{i}\left(\boldsymbol{Y}_{1}^{n} F_{1} \ldots F_{n}\right) \ldots\left(\boldsymbol{Y}_{n}^{n} F_{1} \ldots F_{n}\right)$. However, our translation uses modified expressions, as shown below.

Consider the expression $\left(\boldsymbol{Y}_{i}^{n} F_{1} \ldots F_{n}\right)$. After expanding the notation for $\boldsymbol{Y}_{i}^{n}$ we obtain the expression $\left(\left(\lambda f_{1}, \ldots, f_{n} .\left(X_{i} X_{1} \ldots X_{n}\right)\right) F_{1} \ldots F_{n}\right)$ where $X_{i}$ can be expanded to $X_{i}=\lambda x_{1} \ldots x_{n} \cdot\left(f_{i}\left(x_{1} x_{1} \ldots x_{n}\right) \ldots\left(x_{n} x_{1} \ldots x_{n}\right)\right)$. If we reduce further then we get:

$$
\begin{aligned}
& \left(\lambda f_{1}, \ldots, f_{n} .\left(X_{i} X_{1} \ldots X_{n}\right)\right) F_{1} \ldots F_{n} \stackrel{\text { nbeta,* }}{\longrightarrow}\left(X_{i}^{\prime} X_{1}^{\prime} \ldots X_{n}^{\prime}\right) \text {, } \\
& \text { where } X_{i}^{\prime}=\lambda x_{1} \ldots x_{n} \cdot\left(F_{i}\left(x_{1} x_{1} \ldots x_{n}\right) \ldots\left(x_{n} x_{1} \ldots x_{n}\right)\right)
\end{aligned}
$$

We take the latter expression as the definition of the multi-fixpoint translation, where we avoid substitutions and instead generate (nbeta)-redexes which ensures that contexts are mapped to contexts

Definition 6.2. The translation $N: L_{n a m e} \rightarrow L_{l c c}$ is recursively defined as:

- $N\left(\right.$ letrec $x_{1}=s_{1}, \ldots, x_{n}=s_{n}$ in $\left.t\right)=$

$\left(\lambda x_{1}^{\prime}, \ldots, x_{n}^{\prime} .\left(\lambda x_{1}, \ldots x_{n} . N(t)\right) U_{1} \ldots U_{n}\right) X_{1}^{\prime} \ldots X_{n}^{\prime}$

where $x_{1}^{\prime}, \ldots, x_{n}^{\prime}$ are fresh variables

$U_{i}=x_{i}^{\prime} x_{1}^{\prime} \ldots x_{n}^{\prime}$,

$X_{i}^{\prime}=\lambda x_{1} \ldots x_{n} . F_{i}\left(x_{1} x_{1} \ldots x_{n}\right) \ldots\left(x_{n} x_{1} \ldots x_{n}\right)$,

$F_{i}=\lambda x_{1}, \ldots, x_{n} \cdot N\left(s_{i}\right)$.

- $N(s t)=(N(s) N(t))$

- $N($ seq $s t)=($ seq $N(s) N(t))$

- $N\left(\begin{array}{cccc}c & s_{1} & \ldots & \left.s_{\operatorname{ar}(c)}\right)=\left(c N\left(s_{1}\right) \ldots N\left(s_{\operatorname{ar}(c)}\right)\right)\end{array}\right.$

- $N(\lambda x . s)=\lambda x . N(s)$ 
- $N\left(\operatorname{case}_{T} s\right.$ of alt a $\left._{1} \ldots a l t_{|T|}\right)=\operatorname{case}_{T} N(s)$ of $N\left(\right.$ alt $\left._{1}\right) \ldots N\left(\right.$ alt $\left._{|T|}\right)$

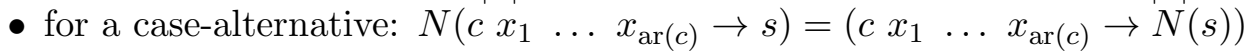

- $N(x)=x$.

We extend $N$ to contexts by treating the hole as a constant, i.e. $N([\cdot])=[\cdot]$. This is consistent, since the hole is not duplicated by the translation.

6.1. Convergence Equivalence of $N$. In the following we will also use the context class $\mathcal{B}$, defined as $\mathcal{B}=L[\mathcal{B}]|A[\mathcal{B}]|[\cdot](\mathcal{L}$ - and $\mathcal{A}$-contexts are defined as before in Sect. 3.2) .

The proof of convergence equivalence of the translation $N$ may be performed directly, but it would be complicated due to the additional (nbeta)-reductions required in $L_{l c c}$. For this technical reason we provide a second translation $N^{\prime}$, which requires a special treatment for the translation of contexts and uses a substitution function $\sigma$ :

Definition 6.3. The translation $N^{\prime}: L_{\text {name }} \rightarrow L_{l c c}$ is recursively defined as:

- $N^{\prime}\left(\right.$ letrec $x_{1}=s_{1}, \ldots, x_{n}=s_{n}$ in $\left.t\right)=\sigma\left(N^{\prime}(t)\right)$, where

$$
\begin{aligned}
\sigma & =\left\{x_{1} \mapsto U_{1}, \ldots x_{n} \mapsto U_{n}\right\} \\
U_{i} & =\left(X_{i}^{\prime} X_{1}^{\prime} \ldots X_{n}^{\prime}\right), \\
X_{i}^{\prime} & =\lambda x_{1} \ldots x_{n} . F_{i}\left(x_{1} x_{1} \ldots x_{n}\right) \ldots\left(x_{n} x_{1} \ldots x_{n}\right), \\
F_{i} & =\lambda x_{1}, \ldots, x_{n} . N^{\prime}\left(s_{i}\right) .
\end{aligned}
$$

- $N^{\prime}(s t)=\left(N^{\prime}(s) N^{\prime}(t)\right)$

- $N^{\prime}($ seq $s t)=\left(\operatorname{seq} N^{\prime}(s) N^{\prime}(t)\right)$

- $N^{\prime}\left(c s_{1} \ldots s_{n}\right)=\left(c N^{\prime}\left(s_{1}\right) \ldots N^{\prime}\left(s_{n}\right)\right)$

- $N^{\prime}(\lambda x . s)=\lambda x \cdot N^{\prime}(s)$

- $N^{\prime}\left(\operatorname{case}_{T} s\right.$ of alt $\ldots$ alt $\left.\operatorname{arT}_{|T|}\right)=\operatorname{case}_{T} N^{\prime}(s)$ of $N^{\prime}\left(a_{1} t_{1}\right) \ldots N^{\prime}\left(\right.$ alt $\left._{|T|}\right)$

- for a case-alternative: $N^{\prime}\left(\begin{array}{lllll}c & x_{1} & \ldots & x_{\operatorname{ar}(c)} \rightarrow s\end{array}\right)=\left(\begin{array}{llll}c & x_{1} & \ldots & x_{\operatorname{ar}(c)} \rightarrow N^{\prime}(s)\end{array}\right)$

- $N^{\prime}(x)=x$.

The extension of $N^{\prime}$ to contexts is done only for $\mathcal{B}$-contexts and requires an extended notion of contexts that are accompanied by an additional substitution, i.e. a $\mathcal{B}$-context translates into a pair $(C, \sigma)$ of a context $C$ and a substitution $\sigma$ acting as a function on expressions. Filling the hole of $(C, \sigma)$ by an expression $s$ is by definition $(C, \sigma)(s)=C[\sigma(s)]$. The translation for $\mathcal{B}$-contexts is defined as

$N^{\prime}(C)=\left(C^{\prime}, \sigma\right)$, where $C^{\prime}$ and $\sigma$ are calculated by applying $N^{\prime}$ to $C$ : for calculating $C^{\prime}$ the hole of $C$ is treated as a constant, and $\sigma$ is the combined substitution affecting the hole of $C^{\prime}$.

This translation does not duplicate holes of contexts.

Lemma 6.4. The translation $N$ is equivalent to $N^{\prime}$ on expressions, that is for all $\mathbb{E}_{\mathcal{L}^{-}}$ expressions $s$ the equivalence $N(s) \sim_{l c c} N^{\prime}(s)$ holds.

Proof. This follows from the definitions and correctness of (nbeta)-reduction in $L_{l c c}$ by Theorem 4.31

We first prove that the translation $N^{\prime}$ is convergence-equivalent. Due to Lemma 6.4 this will also imply that $N$ is convergence-equivalent. All reduction contexts $L[A[\cdot]]$ in $L_{\text {name }}$ translate into reduction contexts $R_{l c c}$ in $L_{l c c}$ since removing the case of letrec from the definition of a reduction context in $L_{n a m e}$ results in the reduction context definition in $L_{l c c}$. 
However, this cannot be reversed, since a translated expression of $L_{\text {name }}$ may have a redex in $L_{l c c}$, but it is not a normal order redex in $L_{\text {name }}$ since (lapp), (lseq), or (lcase) reductions must be performed first to shift letrec-expressions out of an application, a seq-expression, or a case-expression. The lemma below gives a more precise characterization of this relation:

Lemma 6.5. If $L[A[\cdot]]$ is a reduction context in $L_{\text {name }}$, then $N^{\prime}(L[A[\cdot]])=R[\sigma(\cdot)]$, where $R$ is a reduction context in $L_{l c c}$ and $\sigma$ is a substitution.

If $R$ is a reduction context in $L_{l c c}$, and $N^{\prime}\left(C^{\prime}\right)=(R, \sigma)$ for some substitution $\sigma$ and some context $C^{\prime}$ in $L_{\text {name }}$, then $C^{\prime}$ is a $\mathcal{B}$-context.

Proof. The first claim can be shown by structural induction on the context $L[A[\cdot]]$. It holds, since applications are translated into applications, seq-expressions are translated into seqexpressions, case-expressions are translated into case-expressions, and letrec-expressions are translated into substitutions.

The other part can be shown by induction on the number of translation steps. It is easy to observe that the definition of a reduction context in $L_{\text {name }}$ does not descend into letrec-expressions below applications, seq-, and case-expressions. For instance, in ((letrec Env in $\left.\left.\left(\left(\lambda x . s_{1}\right) s_{2}\right)\right) s_{3}\right)$ the reduction contexts are [.] and ([.] $\left.s_{3}\right)$ and the redex is (lapp), i.e. the reduction context does not reach $\left(\left(\lambda x . s_{1}\right) s_{2}\right)$. In general, applications, seq-, and case-expressions in such cases appear in $\mathcal{B}$-contexts, as defined above. By examining the expression definition we observe that these (lapp), (lseq), and/or (lcase)-redexes are the only cases where non-reduction contexts may be translated into reduction contexts.

Lemma 6.6. Let $N^{\prime}(s)=t$. Then:

(1) If $s$ is an abstraction then so is $t$.

(2) If $s=\left(c s_{1} \ldots s_{\operatorname{ar}(c)}\right)$ then $t=\left(c t_{1}^{\prime} \ldots t_{\operatorname{ar}(c)}^{\prime}\right)$.

Proof. This follows by examining the translation $N^{\prime}$.

We will now use reduction diagrams to show the correspondence of $L_{\text {name }}$-reduction and $L_{l c c}$-reduction w.r.t. the translation $N^{\prime}$.

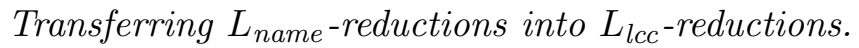

In this section we analyze how normal order reduction in $L_{n a m e}$ can be transferred into $L_{l c c}$ via $N^{\prime}$. We illustrate this by using reduction diagrams. For $s \stackrel{\text { name }}{\longrightarrow} t$ we analyze how the reduction transfers to $N^{\prime}(s)$. The cases are on the rule used in $s \stackrel{\text { name }}{\longrightarrow} t$ :

- (beta) Let $s=R\left[\left(\lambda x . s_{1}\right) s_{2}\right]$ be an expression in $L_{\text {name }}$, where $R$ is a reduction context. We observe that in $L_{n a m e}: s \stackrel{\text { name }}{\longrightarrow} t=R\left[s_{1}\left[s_{2} / x\right]\right]$. Let $N^{\prime}(R[\cdot])=\left(R^{\prime}, \sigma\right)$. Then the translations for $s$ and $t$ are as follows:

$$
\begin{aligned}
& N^{\prime}(s)=R^{\prime}\left[\sigma\left(N^{\prime}\left(\left(\lambda x . s_{1}\right) s_{2}\right)\right)\right]=R^{\prime}\left[\left(\lambda x . \sigma\left(N^{\prime}\left(s_{1}\right)\right)\right) \sigma\left(N^{\prime}\left(s_{2}\right)\right)\right] \\
& N^{\prime}(t)=N^{\prime}\left(R\left[s_{1}\left[s_{2} / x\right]\right]\right)=R^{\prime}\left[\sigma\left(N^{\prime}\left(s_{1}\left[s_{2} / x\right]\right)\right)\right]=R^{\prime}\left[\sigma\left(N^{\prime}\left(s_{1}\right)\right)\left[\sigma\left(N^{\prime}\left(s_{2}\right)\right) / x\right]\right]
\end{aligned}
$$

Since $R^{\prime}$ is a reduction context in $L_{l c c}$, this shows $N^{\prime}(s) \stackrel{l c c, \text { nbeta }}{\longrightarrow} N^{\prime}(t)$. Thus we have the diagram (1) in Figure 10.

- (gcp) Consider the (gcp) reduction. Without loss of generality we assume that $x_{1}$ is the variable that gets substituted:

$$
\begin{aligned}
& s=L\left[\operatorname{letrec} x_{1}=s_{1}, \ldots, x_{n}=s_{n} \text { in } R\left[x_{1}\right]\right] \stackrel{\text { name, }, \mathrm{gcp}}{\longrightarrow} \\
& t=L\left[\operatorname{letrec} x_{1}=s_{1}, \ldots, x_{n}=s_{n} \text { in } R\left[s_{1}\right]\right]
\end{aligned}
$$




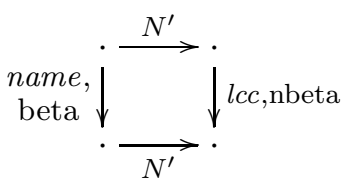

(1)

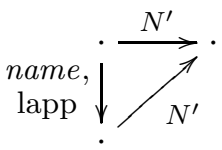

(4)

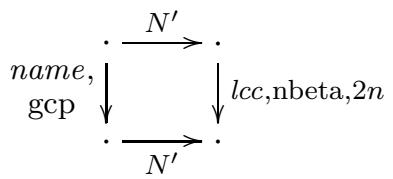

(2)

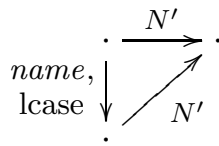

$(5)$

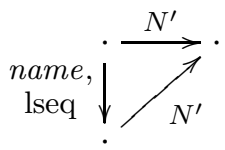

(6)

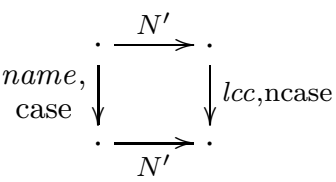

(3)

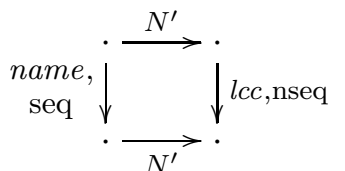

$(7)$

Figure 10: Diagrams for transferring reductions between $L_{n a m e}$ and $L_{l c c}$

Let $N^{\prime}(L)=\left([\cdot], \sigma_{L}\right), N^{\prime}\left(\right.$ letrec $x_{1}=s_{1}, \ldots, x_{n}=s_{n}$ in $\left.[\cdot]\right)=\left([\cdot], \sigma_{E n v}\right)$, and $N^{\prime}(R)=$ $\left(R^{\prime}, \sigma_{R}\right)$ where $R^{\prime}$ is a reduction context. Then

$$
\begin{aligned}
N^{\prime}(s) & =\sigma_{L}\left(\sigma_{E n v}\left(R^{\prime}\left[\sigma_{R}\left(x_{1}\right)\right]\right)\right)=\sigma_{L}\left(\sigma_{E n v}\left(R^{\prime}\right)\right)\left[\sigma_{L}\left(\sigma_{E n v}\left(\sigma_{R}\left(x_{1}\right)\right)\right)\right] \\
& =\sigma_{L}\left(\sigma_{E n v}\left(R^{\prime}\right)\right)\left[\sigma_{L}\left(\sigma_{E n v}\left(x_{1}\right)\right)\right]
\end{aligned}
$$

where the last step follows, since $x_{1}$ cannot be substituted by $\sigma_{R}$, and

$$
N^{\prime}(t)=\sigma_{L}\left(\sigma_{E n v}\left(R^{\prime}\right)\right)\left[\sigma_{L}\left(\sigma_{E n v}\left(N^{\prime}\left(s_{1}\right)\right)\right)\right]
$$

where it is again necessary to observe that $\sigma_{R}\left(s_{1}\right)=s_{1}$ must hold. The context $R^{\prime \prime}=$ $\sigma_{L}\left(\sigma_{E n v}\left(R^{\prime}\right)\right)$ must be a reduction context, since $R^{\prime}$ is a reduction context. This means that we need to show that $R^{\prime \prime}\left[\sigma_{L}\left(\sigma_{E n v}\left(x_{1}\right)\right)\right] \stackrel{l c c, *}{\longrightarrow} R^{\prime \prime}\left[\sigma_{L}\left(\sigma_{E n v}\left(N^{\prime}\left(s_{1}\right)\right)\right)\right]$ holds.

By Definition 6.3 of the translation $N^{\prime}$ we have $\sigma_{L}\left(\sigma_{E n v}\left(x_{1}\right)\right)=U_{1}=\left(X_{1}^{\prime} X_{1}^{\prime} \ldots X_{n}^{\prime}\right)$, where $X_{i}^{\prime}=\lambda x_{1} \ldots x_{n} . F_{i}\left(x_{1} x_{1} \ldots x_{n}\right) \ldots\left(x_{n} x_{1} \ldots x_{n}\right)$, and $F_{i}=\lambda x_{1}, \ldots, x_{n} \cdot \sigma_{L}\left(N^{\prime}\left(s_{i}\right)\right)$, i.e., $N^{\prime}(t)=R^{\prime \prime}\left[U_{1}\right]$.

Performing the applications, we transform $U_{1}$ in $2 n$ steps as

$$
\begin{array}{ll} 
& \left(\lambda x_{1}, \ldots, x_{n} \cdot\left(F_{1}\left(x_{1} x_{1} \ldots x_{n}\right) \ldots\left(x_{n} x_{1} \ldots x_{n}\right)\right)\right) X_{1}^{\prime} \ldots X_{n}^{\prime} \\
\stackrel{\text { nbeta }, n}{\longrightarrow} & F_{1}\left(X_{1}^{\prime} X_{1}^{\prime} \ldots X_{n}^{\prime}\right) \ldots\left(X_{n}^{\prime} X_{1}^{\prime} \ldots X_{n}^{\prime}\right) \\
= & \left(\lambda x_{1}, \ldots, x_{n} \cdot \sigma_{L}\left(N^{\prime}\left(s_{1}\right)\right)\right)\left(X_{1}^{\prime} X_{1}^{\prime} \ldots X_{n}^{\prime}\right) \ldots\left(X_{n}^{\prime} X_{1}^{\prime} \ldots X_{n}^{\prime}\right) \\
\stackrel{\text { nbeta }, n}{\longrightarrow} & \sigma_{L}\left(N^{\prime}\left(s_{1}\right)\right)\left[U_{1} / x_{1}, \ldots, U_{n} / x_{n}\right] .
\end{array}
$$

Obviously, for all reduction contexts in $L_{l c c}$ holds: $r_{1} \stackrel{l c c}{\longrightarrow} r_{2}$ implies $R\left[r_{1}\right] \stackrel{l c c}{\longrightarrow} R\left[r_{1}\right]$. Hence $N^{\prime}(s) \stackrel{l c c, \text { nbeta, } 2 n}{\longrightarrow} R^{\prime \prime}\left[\sigma_{L}\left(N^{\prime}\left(s_{1}\right)\right)\left[U_{1} / x_{1}, \ldots, U_{n} / x_{n}\right]\right]$ and since $x_{1}, \ldots, x_{n}$ cannot occur free in $L$, the last expression is the same as $R^{\prime \prime}\left[\sigma_{L}\left(\sigma_{E n v}\left(N^{\prime}(s)\right)\right)\right]$. Thus we obtain the diagram (2) in Figure 10, where $n$ is the number of bindings in the letrec-subexpression where the copied binding is.

- (case) The diagram for this case is marked (3) in Figure 10, The case is similar to (beta): $s=R\left[\operatorname{case}_{T}\left(c \overrightarrow{s_{i}}\right) \ldots\left(\left(c \overrightarrow{x_{i}}\right) \rightarrow r\right) \ldots\right] \stackrel{\text { name }}{\longrightarrow} R\left[r\left[s_{1} / x_{1}, \ldots, s_{\operatorname{ar}(c)} / x_{\operatorname{ar}(c)}\right]\right]=t$. Let $N^{\prime}(R[\cdot])=\left(R^{\prime}, \sigma\right)$. Then the translations for $s$ and $t$ are as follows:

$$
\begin{aligned}
N^{\prime}(s) & =R^{\prime}\left[\sigma\left(N^{\prime}\left(\operatorname{case}_{T}\left(c s_{1} \ldots s_{\operatorname{ar}(c)}\right) \ldots\left(\left(c x_{1} \ldots x_{\operatorname{ar}(c)}\right) \rightarrow r\right) \ldots\right)\right)\right] \\
& =R^{\prime}\left[\operatorname{case}_{T}\left(c \sigma\left(N^{\prime}\left(s_{1}\right)\right)\right) \ldots \sigma\left(N^{\prime}\left(s_{\operatorname{ar}(c)}\right)\right) \ldots\left(\left(c x_{1} \ldots x_{\operatorname{ar}(c)}\right) \rightarrow \sigma\left(N^{\prime}(r)\right)\right) \ldots\right]
\end{aligned}
$$




$$
\begin{aligned}
N^{\prime}(t) & =N^{\prime}\left(R\left[r\left[s_{1} / x_{1}, \ldots, s_{\operatorname{ar}(c)} / x_{\operatorname{ar}(c)}\right]\right]\right) \\
& =R^{\prime}\left[\sigma\left(N^{\prime}\left(r\left[s_{1} / x_{1}, \ldots, s_{\operatorname{ar}(c)} / x_{\operatorname{ar}(c)}\right]\right)\right)\right] \\
& =R^{\prime}\left[\sigma\left(N^{\prime}(r)\right)\left[\sigma\left(N^{\prime}\left(s_{1}\right)\right) / x_{1}, \ldots, \sigma\left(N^{\prime}\left(s_{\operatorname{ar}(c)}\right)\right) / x_{\operatorname{ar}(c)}\right]\right]
\end{aligned}
$$

Since $R^{\prime}$ is a reduction context in $L_{l c c}$, this shows $N^{\prime}(s) \stackrel{l c c}{\longrightarrow} N^{\prime}(t)$.

- (lapp) The reduction is $R\left[\left(\right.\right.$ letrec Env in $\left.\left.s_{1}\right) s_{2}\right] \stackrel{\text { name }}{\longrightarrow} R\left[\left(\right.\right.$ letrec Env in $\left.\left.\left(s_{1} s_{2}\right)\right)\right]$. Since free variables of $s_{2}$ do not depend on $E n v$, the translation of $s_{2}$ does not change by adding Env. I.e., for $N^{\prime}(R)=\left(R^{\prime}, \sigma_{R}\right)$ and $N^{\prime}($ letrec Env in $[\cdot])=\left([\cdot], \sigma_{E n v}\right)$ we have

$$
\begin{aligned}
N^{\prime}\left(R\left[\left(\text { letrec Env } \in s_{1}\right) s_{2}\right]\right) & =R^{\prime}\left[\sigma_{R}\left(\sigma_{E n v}\left(N^{\prime}\left(s_{1}\right)\right) N^{\prime}\left(s_{2}\right)\right)\right] \\
& =R^{\prime}\left[\sigma_{R}\left(\sigma_{E n v}\left(N^{\prime}\left(s_{1} N^{\prime}\left(s_{2}\right)\right)\right)\right)\right] \\
& =N^{\prime}\left(R\left[\left(\text { letrec Env in }\left(s_{1} s_{2}\right)\right)\right]\right)
\end{aligned}
$$

and thus the diagram for this case is as the one marked (4) in Figure 10.

- (lcase) The case is analogous to that of (lapp), with the diagram marked as (5) in Figure 10

- (lseq) The case is analogous to (lapp) and (lcase), with the diagram (6) in Figure 10.

- (seq) $s=R\left[\operatorname{seq} v s_{1}\right] \stackrel{\text { name }}{\longrightarrow} R\left[s_{1}\right]=t$ where $v$ is an abstraction or a constructor application

Let $N^{\prime}(R[\cdot])=\left(R^{\prime}, \sigma\right)$. Then the translations for $s$ and $t$ are as follows:

$$
\begin{aligned}
& N^{\prime}(s)=R^{\prime}\left[\sigma\left(N^{\prime}\left(\operatorname{seq} v s_{1}\right)\right)\right]=R^{\prime}\left[\operatorname{seq} \sigma\left(N^{\prime}(v)\right) \sigma\left(N^{\prime}\left(s_{1}\right)\right)\right] \\
& N^{\prime}(t)=R^{\prime}\left[\sigma\left(N^{\prime}\left(s_{1}\right)\right)\right]
\end{aligned}
$$

By Lemma 6.6 $N^{\prime}(v)$ is a value in $L_{l c c}$ (which cannot be changed by the substitution $\sigma)$ and thus $N^{\prime}(s) \stackrel{l c c, \text { nseq }}{\longrightarrow} N^{\prime}(t)$. The diagram for this case is (7) in Figure 10,

We inspect how WHNFs and values of both calculi are related w.r.t. $N^{\prime}$ :

Lemma 6.7. Let $s$ be irreducible in $L_{n a m e}$, but not an $L_{n a m e}-W H N F$. Then $N^{\prime}(s)$ is irreducible in $L_{l c c}$ and also not an $L_{l c c}-W H N F$.

Proof. Assume that expression $s$ is irreducible in $L_{\text {name }}$ but not an $L_{\text {name }}$-WHNF. There are three cases:

(1) Expression $s$ is of the form $R[x]$ where $x$ is a free variable in $R[x]$, then let $N^{\prime}(R)=$ $\left(R^{\prime}, \sigma\right)$ and thus $N^{\prime}(s)=R^{\prime}[\sigma(x)]$. Since $\sigma$ only substitutes bound variables, we get $\sigma(x)=x$ and thus $N^{\prime}(s)=R^{\prime}[x]$ where $x$ is free in $R^{\prime}[x]$. Hence $N^{\prime}(s)$ cannot be an $L_{l c c}-\mathrm{WHNF}$ and it is irreducible in $L_{l c c}$.

(2) Expression $s$ is of the form $R\left[\operatorname{case}_{T}\left(\begin{array}{cccc}c & s_{1} & \ldots & \left.s_{\operatorname{ar}(c)}\right)\end{array}\right)\right.$ of alts], but $c$ is not of type $T$. Let $N^{\prime}(R)=\left(R^{\prime}, \sigma\right)$. Then $N^{\prime}(s)=R^{\prime}\left[\operatorname{case}_{T}\left(c \sigma\left(N^{\prime}\left(s_{1}\right)\right) \ldots \sigma\left(N^{\prime}\left(s_{\operatorname{ar}(c)}\right)\right)\right)\right.$ of alts $]$ which shows that $N^{\prime}(s)$ is not an $L_{l c c}$ WHNF and irreducible in $L_{l c c}$.

(3) Expression $s$ is of the form $R\left[\left(\left(\begin{array}{llll}c & s_{1} & \ldots & \left.s_{\operatorname{ar}(c)}\right) r\end{array}\right)\right]\right.$. Then again $N^{\prime}(s)$ is not an $L_{l c c^{-}}$ WHNF and irreducible.

Lemma 6.8. Let $s \in \mathbb{E}_{\mathcal{L}}$. Then $s$ is an $L_{\text {name }}-W H N F$ iff $N^{\prime}(s)$ is an $L_{l c c}-W H N F$.

Proof. If $s=L\left[\lambda x . s^{\prime}\right]$ or $s=L\left[\left(c s_{1} \ldots s_{\operatorname{ar}(c)}\right)\right]$ then $N^{\prime}(s)=\lambda x \cdot \sigma\left(N^{\prime}\left(s^{\prime}\right)\right)$ or $N^{\prime}(s)=$ $\left(c \sigma\left(N^{\prime}\left(s_{1}\right)\right) \ldots \sigma\left(N^{\prime}\left(s_{\operatorname{ar}(c)}\right)\right)\right)$ respectively, both of which are $L_{l c c}$-WHNFs.

For the other direction assume that $N^{\prime}(s)$ is an abstraction or a constructor application. The analysis of the reduction correspondence in the previous paragraph shows that $s$ cannot have a normal order redex in $L_{n a m e}$, since otherwise $N^{\prime}(s)$ cannot be an $L_{l c c}$-WHNF. Lemma 
6.7 shows that $s$ cannot be irreducible in $L_{\text {name }}$, but not an $L_{n a m e}$-WHNF. Thus $s$ must be an $L_{n a m e}$-WHNF.

Transferring $L_{l c c}$-reductions into $L_{\text {name-reductions. }}$

We will now analyze how normal order reductions for $N^{\prime}(s)$ can be transferred into normal order reductions for $s$ in $L_{\text {name }}$.

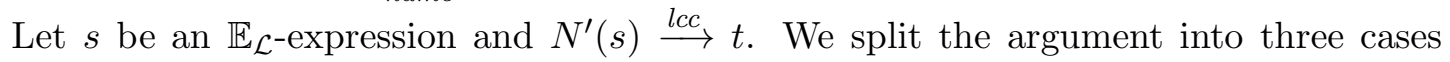
based on whether or not a normal order reduction is applicable to $s$ :

- If $s \stackrel{(\text { name })}{\longrightarrow} r$, then we can use the already developed diagrams, since normal-order reduction in both calculi is unique.

- $s$ is a WHNF. This case cannot happen, since then $N^{\prime}(s)$ would also be a WHNF (see Lemma 6.8) and thus irreducible.

- $s$ is irreducible but not a WHNF. Then Lemma 6.7 implies that $N^{\prime}(s)$ is irreducible in $L_{l c c}$ which contradicts the assumption $N^{\prime}(s) \stackrel{l c c}{\longrightarrow} t$. Thus this case is impossible.

We summarize the diagrams in the following lemma:

Lemma 6.9. Normal-order reductions in $L_{n a m e}$ can be transferred into reductions in $L_{l c c}$, and vice versa, by the diagrams in Figure 10.

Proposition 6.10. $N^{\prime}$ and $N$ are convergence equivalent, i.e. for all $\mathbb{E}_{\mathcal{L}}$-expressions $s$ : $s \downarrow_{\text {name }} \Longleftrightarrow N^{\prime}(s) \downarrow_{l c c}\left(s \downarrow_{\text {name }} \Longleftrightarrow N(s) \downarrow_{l c c}\right.$, resp. $)$.

Proof. We first prove convergence equivalence of $N^{\prime}$ : Suppose $s \downarrow_{n a m e}$. Let $s \stackrel{\text { name,k }}{\longrightarrow} s_{1}$

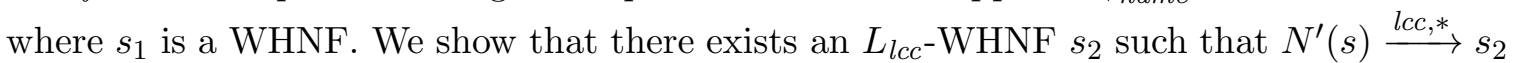
by induction on $k$. The base case follows from Lemma 6.8. The induction step follows by applying a diagram from Lemma 6.9 and then using the induction hypothesis.

For the other direction we assume that $N^{\prime}(s) \downarrow_{l c c}$, i.e. there exists a WHNF $s_{1} \in L_{l c c}$ s.t. $N^{\prime}(s) \stackrel{l c c, k}{\longrightarrow} s_{1}$. By induction on $k$ we show that there exists a $L_{n a m e}-\mathrm{WHNF} s_{2}$ such that $s \stackrel{\text { name,* }}{\longrightarrow} s_{2}$. The base case is covered by Lemma 6.8. The induction step uses the diagrams. Here it is necessary to observe that the diagrams for the reductions (lapp), (lcase), and (lseq) cannot be applied infinitely often without being interleaved with other reductions. This holds, since let-shifting by (lapp), (lcase), and (lseq) moves letrec-symbols to the top of the expressions, and thus there are no infinite sequences of these reductions.

It remains to show convergence equivalence of $N$ : Let $s \downarrow_{\text {name }}$ then $N^{\prime}(s) \downarrow_{l c c}$, since $N^{\prime}$ is convergence equivalent. Lemma 6.4 implies $N^{\prime}(s) \sim_{l c c} N(s)$ and thus $N(s) \downarrow_{l c c}$ must hold. For the other direction Lemma 6.4 shows that $N(s) \downarrow_{l c c}$ implies $N^{\prime}(s) \downarrow_{l c c}$. Using convergence equivalence of $N^{\prime}$ yields $s \downarrow_{\text {name }}$.

Lemma 6.11. The translation $N$ is compositional, i.e. for all expressions $s$ and all contexts $C: N(C[s])=N(C)[N(s)]$.

Proof. This easily follows by structural induction on the definition.

Proposition 6.12. For all $s_{1}, s_{2} \in \mathbb{E}_{\mathcal{L}}: N\left(s_{1}\right) \leq_{l c c} N\left(s_{2}\right) \Longrightarrow s_{1} \leq_{\text {name }} s_{2}$, i.e. $N$ is adequate.

Proof. Since $N$ is convergence-equivalent (Proposition 6.10) and compositional by Lemma 6.11, we derive that $N$ is adequate (see [SSNSS08] and Section 2). 
Lemma 6.13. For letrec-free expressions $s_{1}, s_{2} \in \mathbb{E}_{\lambda}$ the following holds: $s_{1} \leq_{\text {name }}$ $s_{2} \Longrightarrow s_{1} \leq_{l c c} s_{2}$.

Proof. Note that the claim only makes sense since clearly $\mathbb{E}_{\lambda} \subseteq \mathbb{E}_{\mathcal{L}}$. Let $s_{1}$, $s_{2}$ be letrec-free such that $s_{1} \leq_{\text {name }} s_{2}$. Let $C$ be an $L_{l c c}$-context such that $C\left[s_{1}\right] \downarrow_{l c c}$, i.e. $C\left[s_{1}\right] \stackrel{l c c, k}{\longrightarrow} \lambda x . s_{1}^{\prime}$. By comparing the reduction strategies in $L_{n a m e}$ and $L_{l c c}$, we obtain that $C\left[s_{1}\right] \stackrel{\text { name,k }}{\longrightarrow} \lambda x . s_{2}^{\prime}$ (by the identical reduction sequence) since $C\left[s_{1}\right]$ is letrec-free. Thus, $C\left[s_{1}\right] \downarrow_{\text {name }}$ and also $C\left[s_{2}\right] \downarrow_{\text {name }}$, i.e. there is a normal order reduction in $L_{\text {name }}$ for $C\left[s_{2}\right]$ to a WHNF. Since $C\left[s_{2}\right]$ is letrec-free, we can perform the identical reduction in $L_{l c c}$ and obtain $C\left[s_{2}\right] \downarrow_{l c c}$.

The language $L_{l c c}$ is embedded into $L_{n a m e}$ (and also $L_{L R}$ ) by $\iota(s)=s$.

Proposition 6.14. For all $s \in \mathbb{E}_{\mathcal{L}}: s \sim_{\text {name }} \iota(N(s))$.

Proof. We first show that for all expressions $s \in \mathbb{E}_{\mathcal{L}}: s \sim_{\text {name }} \iota(N(s))$. Since $N$ is the identity mapping on letrec-free expressions of $L_{\text {name }}$ and $N(s)$ is letrec-free, we have $N(\iota(N(s)))=N(s)$. Hence adequacy of $N$ (Proposition 6.12) implies $s \sim_{\text {name }} \iota(N(s)$ ).

Proposition 6.15. For all $s_{1}, s_{2} \in \mathbb{E}_{\mathcal{L}}: s_{1} \leq_{\text {name }} s_{2} \Longrightarrow N\left(s_{1}\right) \leq_{l c c} N\left(s_{2}\right)$.

Proof. For this proof it is necessary to observe that $\mathbb{E}_{\lambda} \subseteq \mathbb{E}_{\mathcal{L}}$, thus we can treat $L_{l c c}$ expressions as $L_{\text {name }}$ expressions. Let $s_{1}, s_{2} \in \mathbb{E}_{\mathcal{L}}$ and $s_{1} \leq_{\text {name }} s_{2}$. By Proposition 6.14: $N\left(s_{1}\right) \sim_{\text {name }} s_{1} \leq_{\text {name }} s_{2} \sim_{\text {name }} N\left(s_{2}\right)$, thus $N\left(s_{1}\right) \leq_{\text {name }} N\left(s_{2}\right)$. Since $N\left(s_{1}\right)$ and $N\left(s_{2}\right)$ are letrec-free, we can apply Lemma 6.13 and thus have $N\left(s_{1}\right) \leq_{l c c} N\left(s_{2}\right)$.

Now we put all parts together, where $(N \circ W)(s)$ means $N(W(s))$ :

Theorem 6.16. $N$ and $N \circ W$ are fully-abstract, i.e. for all expressions $s_{1}, s_{2} \in \mathbb{E}_{\mathcal{L}}$ : $s_{1} \leq_{L R} s_{2} \Longleftrightarrow N\left(W\left(s_{1}\right)\right) \leq_{l c c} N\left(W\left(s_{2}\right)\right)$.

Proof. Full-abstractness of $N$ follows from Propositions 6.12 and 6.15, Full-abstractness of $N \circ W$ thus holds, since $W$ is fully-abstract (Corollary 5.32).

Since $N$ is surjective, this and Corollary 6.17 imply:

Corollary 6.17. $N$ and $N \circ W$ are isomorphisms according to Definition 2.7.

The results also allow us to transfer the characterization of expressions in $L_{l c c}$ into $L_{L R}$. With $c$ Bot $_{L R}$ we denote the set of $\mathbb{E}_{\mathcal{L}}$-expressions $s$ with the property that for all substitutions $\sigma$ : if $\sigma(s)$ is closed, then $\sigma(s) \Uparrow L R$.

Proposition 6.18. Let $s$ be a closed $\mathbb{E}_{\mathcal{L}}$-expression. Then there are three cases: $s \sim \Omega$, $s \sim_{L R} \lambda x . s^{\prime}$ for some $s^{\prime}, s \sim_{L R} c s_{1} \ldots s_{n}$ for some terms $s_{1}, \ldots, s_{n}$ and constructor $c$.

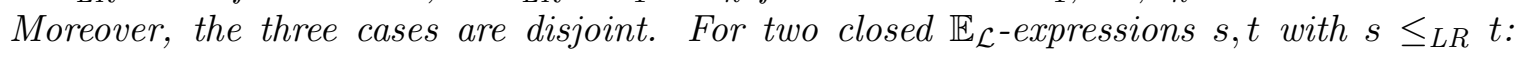
Either $s \sim_{L R} \Omega$, or $s \sim_{L R} c s_{1} \ldots s_{n}, t \sim c t_{1} \ldots t_{n}$ and $s_{i} \leq_{L R} t_{i}$ for all $i$ for some terms $s_{1}, \ldots, s_{n}, t_{1}, \ldots, t_{n}$ and constructor $c$, or $s \sim_{L R} \lambda x . s^{\prime}$ and $t \sim_{L R} \lambda x . t^{\prime}$ for some expressions $s^{\prime}, t^{\prime}$ with $s^{\prime} \leq_{L R} t^{\prime}$, or $s \sim_{L R} \lambda x . s^{\prime}$ and $t \sim_{L R} c t_{1} \ldots t_{n}$ for some term $s^{\prime} \in c B o t_{L R}$, expressions $t_{1}, \ldots, t_{n}$ and constructor $c$.

Proof. This follows by Proposition 4.32 and since $N \circ W$ is surjective, compositional and fully abstract, and the identity on constructors. 


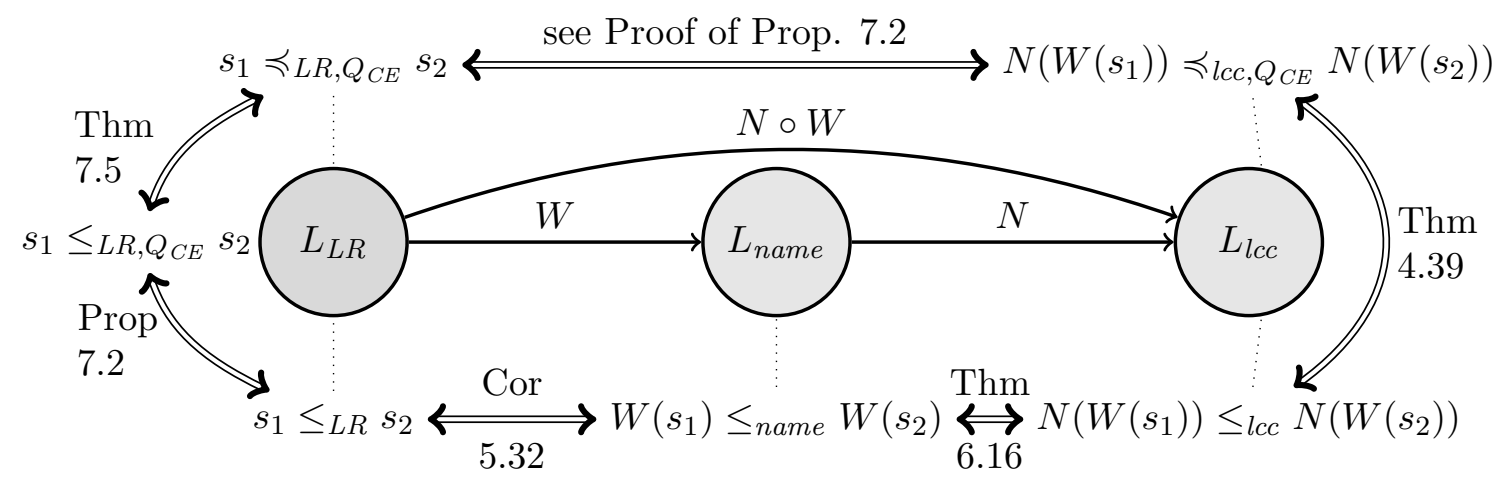

Figure 11: The structure of the reasoning for the similarities in $L_{L R}$ for closed expressions.

\section{On Similarity in $L_{L R}$}

In this section we will explain co-inductive and inductive (bi)similarity for $L_{L R}$. Our results of the previous sections then enable us to show that these bisimilarities coincide with contextual equivalence in $L_{L R}$.

7.1. Overview of soundness and completeness proofs for similarities in $L_{L R}$. Before we give details of the proof for lifting soundness and completeness of similarities from $L_{l c c}$ to $L_{L R}$, we show an outline of the proof in Fig. 11. The diagram shows fully abstract translations between the calculi $L_{L R}, L_{\text {name }}$, and $L_{l c c}$ defined and studied in Sections 5 and 6. where Corollary 5.32 and Theorem 6.16 show full abstractness for $W$ and $N$, respectively. These fully-abstract translations that are also surjective, and the identity on letrec-free expressions, allow us to prove that $s_{1} \leq_{L R} s_{2} \Longleftrightarrow N\left(W\left(s_{1}\right)\right) \leq_{l c c} N\left(W\left(s_{2}\right)\right)$. By Theorem 4.37 in $L_{l c c}$, this is equivalent to $N\left(W\left(s_{1}\right)\right) \preccurlyeq_{l c c}^{o} N\left(W\left(s_{2}\right)\right)$. The proof is completed by using the translations by transferring the equations back and forth between $L_{L R}$ and $L_{l c c}$ in this section in order to finally show that $s_{1} \leq_{L R} s_{2} \Longleftrightarrow s_{1} \preccurlyeq_{L R, Q_{C E}}^{o} s_{2}$ in Theorem 7.6 .

7.2. Similarity in $L_{L R}$. The definition of $L_{L R}$-WHNFs implies that they are of the form

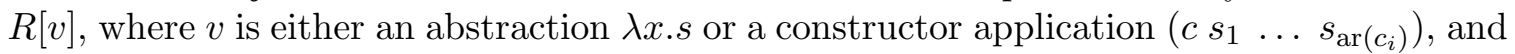
where $R$ is an $L_{L R}-A W H N F$-context according to the grammar $R::=[\cdot] \mid$ (letrec Env in [.]) if $v$ is an abstraction, and $R$ is an $L_{L R}-C W H N F$-context according to the grammar $R::=$ [.] $\mid\left(\right.$ letrec Env in [.]) $\mid\left(\right.$ letrec $x_{1}=[\cdot],\left\{x_{i}=x_{i-1}\right\}_{i=2}^{m}$, Env in $\left.x_{m}\right)$ if $v$ is a constructor application. Note that $L_{L R^{-}} \mathrm{AWHNF}$-contexts and $L_{L R^{-}} \mathrm{CWHNF}$-contexts are special $L_{L R^{-}}$ reduction contexts, also called $L_{L R}-W H N F$-contexts.

First we show that finite simulation (see [SSM08]) is correct for $L_{L R}$ :

Definition 7.1. Let $\leq_{L R, Q_{C E}}$ be defined for $L_{L R}$ as instantiating the relation $\leq_{\mathcal{Q}}$ in Definition 2.6 with the closed subcalculus of the calculus $L_{L R}$ and the set $\mathcal{Q}$ with $Q_{C E}$ from Definition 4.38 .

The relation $\preccurlyeq L R, Q_{C E}$ is $\mathcal{Q}$-similarity (Definition 2.5) instantiated for the calculus $L_{L R}$ with the set of contexts $Q_{C E}$ (Definition 4.38). Its open extension is denoted with $\preccurlyeq_{L R, Q_{C E}}^{o}$.

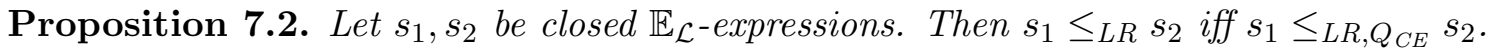


Proof. The $\Rightarrow$ direction is trivial. We show $\Leftarrow$, the nontrivial part: Assume that the inequation $s_{1} \leq_{L R, Q_{C E}} s_{2}$ holds. Then $N\left(W\left(s_{1}\right)\right) \leq_{l c c, Q_{C E}} N\left(W\left(s_{2}\right)\right)$, since for every $n \geq 0$ and context $Q=Q_{n}\left(\ldots\left(Q_{2}\left(Q_{1}[]\right) \ldots\right)\right)$ with $Q_{i} \in Q_{C E}$, we have $N(W(Q))=Q$, and also $Q\left(s_{i}\right) \downarrow_{L R} \Longleftrightarrow Q\left(s_{i}\right) \downarrow_{l c c}$, since $N \circ W$ is convergence-equivalent and compositional, and the identity on letrec-free expressions. Now Theorem 4.39 shows $N\left(W\left(s_{1}\right)\right) \leq_{l c c} N\left(W\left(s_{2}\right)\right)$, and then Theorem 6.16 shows $s_{1} \leq_{L R} s_{2}$.

The following lemma is helpful in applying Theorem 4.8.

Lemma 7.3. The closed part of the calculus $L_{L R}$ is convergence-admissible: For all contexts $Q \in Q_{C E}$, and closed $L_{L R^{-}}$WHNFs $w: Q(s) \downarrow_{L R} w$ iff $\exists v: s \downarrow_{L R} v$ and $Q(v) \downarrow_{L R} w$.

Proof. " $\Rightarrow$ ": First assume $Q$ is of the form $([\cdot] r)$ for closed $r$. Let $(s r) \downarrow_{L R} w$. There are two cases, which can be verified by induction on the length $k$ of a reduction sequence $(s r) \stackrel{L R, k}{\longrightarrow}$ $w:(s r) \stackrel{L R, *}{\longrightarrow}\left(\left(\lambda x . s^{\prime}\right) r\right) \stackrel{L R, *}{\longrightarrow} w$, where $s \stackrel{L R, *}{\longrightarrow}\left(\lambda x \cdot s^{\prime}\right)$, and the claim holds. The other case is $(s r) \stackrel{L R, *}{\longrightarrow}\left(\right.$ letrec Env in $\left.\left(\left(\lambda x . s^{\prime}\right) r\right)\right) \stackrel{L R, *}{\longrightarrow} w$, where $s \stackrel{L R, *}{\longrightarrow}\left(\right.$ letrec Env in $\left.\left(\lambda x . s^{\prime}\right)\right)$. In this case $\left(\left(\right.\right.$ letrec Env in $\left.\left.\left(\lambda x . s^{\prime}\right)\right) r\right) \stackrel{L R,(\text { lapp })}{\longrightarrow}\left(\right.$ letrec Env in $\left.\left(\left(\lambda x . s^{\prime}\right) r\right)\right) \stackrel{L R, *}{\longrightarrow} w$, and thus the claim is proven. The other cases where $Q$ is of the form $\left(\operatorname{case}_{T}[\cdot]\right.$ of $\left.\ldots\right)$ can be proven similarly.

The " $\Leftarrow$ "-direction can be proven using induction on the length of reduction sequences.

Lemma 7.4. In $L_{L R}$, the equation $\left(\leq_{L R}^{c}\right)^{o}=\leq_{L R}$ holds.

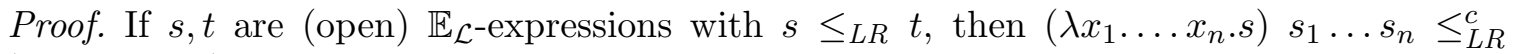
$\left(\lambda x_{1} \ldots x_{n} . t\right) s_{1} \ldots s_{n}$ for closed expressions $s_{i}$, and then by correctness of reduction in $L_{L R}, \sigma(s) \leq_{L R}^{c} \sigma(t)$, and hence $\leq_{L R} \subseteq\left(\leq_{L R}^{c}\right)^{o}$.

If for all closing $\mathbb{E}_{\mathcal{L}}$-substitutions $\sigma: \sigma(s) \leq_{L R}^{c} \sigma(t)$, then using the fully abstract translations $N \circ W$, we obtain $N \circ W(\sigma)(N \circ W(s)) \leq_{l c c}^{c} N \circ W(\sigma)(N \circ W(t))$, hence $N \circ W(s) \leq_{l c c}^{c}(N \circ W(t))$ by Theorem 4.39, Again using fully abstractness of $N \circ W$, we obtain $s \leq_{L R} t$.

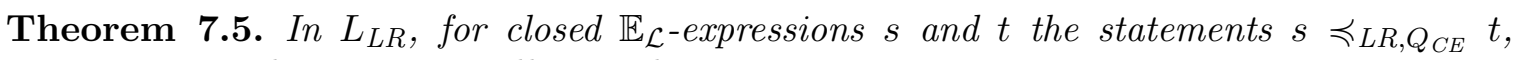
$s \leq_{L R, Q_{C E}} t$ and $s \leq_{L R} t$ are all equivalent.

Proof. Lemma 7.3 shows that Theorem 4.8 is applicable for the testing contexts from $Q_{C E}$, i.e. $\preccurlyeq_{L R, Q_{C E}}=\leq_{L R, Q_{C E}}$ and Proposition 7.2 shows $\leq_{L R, Q_{C E}}=\leq_{L R}^{c}$

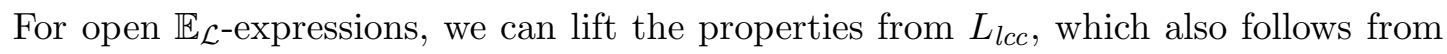
full abstraction of $N \circ W$ and from Lemma 4.40.

The results above imply the following theorem:

Main Theorem 7.6. $\leq_{L R}=\preccurlyeq_{L R, Q_{C E}}^{o}$.

Proof. Theorem 7.5 shows $\preccurlyeq L R, Q_{C E}=\leq_{L R, Q_{C E}}=\leq_{L R}^{c}$, hence $\preccurlyeq_{L R, Q_{C E}}^{o}=\left(\leq_{L R}^{c}\right)^{o}$. Then Lemma 7.4 shows $\left(\leq_{L R}^{c}\right)^{o}=\leq_{L R}=\preccurlyeq_{L R, Q_{C E}}^{o}$.

The Main Theorem 7.6 implies that our embedding of $L_{l c c}$ into the call-by-need letrec calculus $L_{L R}$ (modulo $\sim$ ) is isomorphic w.r.t. the corresponding term models, i.e.

Theorem 7.7. The identical embedding $\iota: \mathbb{E}_{\lambda} \rightarrow \mathbb{E}_{\mathcal{L}}$ is an isomorphism according to Definition 2.7. 
Remark 7.8. Consider a polymorphically typed variant of $L_{L R}$, say $L_{L R}^{\text {poly }}$, and a corresponding type-indexed contextual preorder $\leq_{L R, \text { poly }, \tau}$ which relates expressions of polymorphic type $\tau$ and where the testing contexts are restricted to well-typed contexts, i.e. for $s, t$ of type $\tau$ the inequality $s \leq_{L R \text {,poly }, \tau} t$ holds iff for all contexts $C$ such that $C[s]$ and $C[t]$ are well-typed: $C[s] \downarrow_{L R} \Longrightarrow C[t] \downarrow_{L R}$. Obviously for all expressions $s, t$ of type $\tau$ the inequality $s \leq_{L R} t$ implies $s \leq_{L R \text {,poly, } \tau} t$, since any test (context) performed for $\leq_{L R, \text { poly }, \tau}$ is also included in the tests for $\leq_{L R}$ (there are more contexts). Thus the main theorem

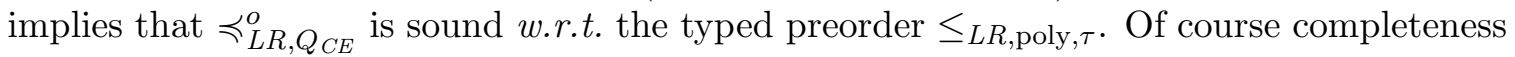
does not hold, and requires another definition of similarity which respects the typing.

7.3. Similarity up to $\sim_{L R}$. A more comfortable tool to prove program equivalences in $L_{L R}$ is the following similarity definition which allows to simplify intermediate expressions that are known to be equivalent.

Definition 7.9 (Similarity up to $\sim_{L R}$ ). Let $\preccurlyeq L R, \sim$ be the greatest fixpoint of the following operator $F_{L R, \sim}$ on closed $\mathbb{E}_{\mathcal{L}^{-} \text {-expressions: }}$

We define an operator $F_{L R, \sim}$ on binary relations $\eta$ on closed $L_{l c c}$-expressions: $s F_{L R, \sim}(\eta) t$ iff the following holds:

(1) If $s \sim_{L R} \lambda x . s^{\prime}$ then there are two possibilities: (i) if $t \sim_{L R}\left(c t_{1} \ldots t_{n}\right)$ then $s^{\prime} \in c B o t_{L R}$, or (ii) if $t \sim_{L R} \lambda x . t^{\prime}$ then for all closed $r:\left(\left(\lambda x . s^{\prime}\right) r\right) \eta\left(\left(\lambda x . t^{\prime}\right) r\right)$;

(2) If $s \sim_{L R}\left(c s_{1} \ldots s_{n}\right)$ then $t \sim_{L R}\left(c t_{1} \ldots t_{n}\right)$ and $s_{i} \eta t_{i}$ for all $i$.

Lemma 7.10. $\leq_{L R}^{c} \subseteq \preccurlyeq L R, \sim$

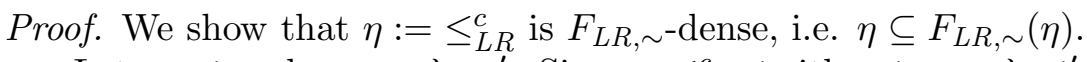

Let $s \eta t$ and $s \sim_{L R} \lambda x . s^{\prime}$. Since $s \leq_{L R}^{c} t$ either $t \sim_{L R} \lambda x$. $t^{\prime}$ or $t \sim_{L R} c t_{1} \ldots t_{n}$ and $s^{\prime} \in c B o t_{L R}$. For the latter case we are finished. For the former case we have $\lambda x . t^{\prime} \sim_{L R}^{c} t$. Since $\leq_{L R}^{c}$ is a precongruence, this implies $\left(\left(\lambda x . s^{\prime}\right) r\right) \leq_{L R}\left(\left(\lambda x . t^{\prime}\right) r\right)$ for all closed $\mathbb{E}_{\mathcal{L}^{-}}$ expressions $r$. Thus we conclude $s F_{L R, \sim}(\eta) t$.

Now let $s \eta t$ and $s \sim_{L R}^{c} c s_{1} \ldots s_{n}$. Then $t \sim_{L R}^{c}\left(c t_{1} \ldots t_{n}\right)$ by Proposition 6.18. The contexts $C_{i}:=\left(\right.$ case [] of $\left.\ldots\left(c x_{1} \ldots x_{n} \rightarrow x_{i}\right) \ldots\right)$ where all other right hand sides of case-alternatives are $\perp$, show that also $s_{i} \leq_{L R} t_{i}$ must hold, since otherwise $s \leq_{L R}^{c} t$ cannot hold. Thus also in this case $s F_{L R, \sim}(\eta) t$ holds.

Lemma 7.11. $N(W(\preccurlyeq L R, \sim)) \subseteq \preccurlyeq l c c, \sim$.

Proof. We show that $\eta:=\{(N(W(s)), N(W(t))) \mid s \preccurlyeq L R, \sim t\}$ is $F_{l c c, \sim}$-dense (see Definition 4.43), i.e. $\eta \subseteq F_{l c c, \sim}(\eta)$. Let $s \preccurlyeq L R, \sim t$ for closed $s, t$. If $N(W(s)) \sim_{l c c} \lambda x . s^{\prime}$, then also $s \sim_{L R} \lambda x . s^{\prime}$. Now there are two cases: If $t \sim_{L R}\left(c t_{1} \ldots t_{n}\right)$ then $s^{\prime} \in c B o t_{L R}$ must hold. Then also $s^{\prime} \in c B o t$ and $N(W(t)) \sim_{l c c}\left(c t_{1} \ldots t_{n}\right)$ and we are finished. If $t \sim_{L R} \lambda x \cdot t^{\prime}$ then for all closed $\mathbb{E}_{\mathcal{L}}$-expressions $r:\left(\lambda x . s^{\prime}\right) r \preccurlyeq L R, \sim\left(\lambda x . t^{\prime}\right) r$ (by unfolding the fixpoint equation for $\left.F_{L R, \sim}\right)$. Since $N \circ W$ is surjective, compositional and fully abstract, this also shows $N\left(W\left(\lambda x . s^{\prime}\right)\right) r \eta N\left(W\left(\lambda x . t^{\prime}\right)\right) r$ for all $L_{l c c}$-expressions $r$.

If $N(W(s)) \sim_{l c c}\left(c s_{1} \ldots s_{n}\right)$, then also $s \sim_{L R}\left(c s_{1} \ldots s_{n}\right)$. Now $s \preccurlyeq L R, \sim t$ shows that $t \sim_{L R}\left(\begin{array}{cccc}c & t_{1} & \ldots & t_{n}\end{array}\right)$ such that for all $i: s_{i} \preccurlyeq_{L R, \sim} t_{i}$. Hence $\left(s_{i}, t_{i}\right) \in \eta$ and also $N(W(t)) \sim_{l c c}\left(c t_{1} \ldots t_{n}\right)$, since $N \circ W$ is fully abstract. 
Theorem 7.12. $\leq_{L R}=\preccurlyeq_{L R, \sim}^{o}$

Proof. For the closed relations, one direction of the equation $\preccurlyeq L R, \sim=\leq_{L R}^{c}$ is Lemma 7.10, the other direction follows from Lemma 7.11, since $s \preccurlyeq L R, \sim t$ implies $N(W(s)) \preccurlyeq l c c, \sim$ $N(W(t))$ which in turn implies $N(W(s)) \leq_{l c c}^{c} N(W(t))$ and finally, full-abstraction of $N \circ W$ shows $s \leq_{L R}^{c} t$.

For the open extension the claimed equality holds, since $s \leq_{L R} t$ iff $\sigma(s) \leq_{L R} \sigma(t)$ for all closing substitutions $\sigma$ : This holds, since for $\sigma=\left\{x_{1} \mapsto s_{1}, \ldots, x_{n} \mapsto s_{n}\right\}$ the equation $\sigma(s) \sim_{L R}$ letrec $x_{1}=s_{1}, \ldots, x_{n}=s_{n}$ in $s$ holds by correctness of the general copy rule (gcp) (Proposition 5.34) and of garbage collection (gc) (Theorem 3.7).

We demonstrate the use of similarity up to $\sim_{L R}$ in the following example:

Example 7.13. As an example we prove the list law $R[$ map $(\lambda x$.True) (repeat $u)] \sim_{L R}$ $R^{\prime}[($ repeat True $)]$ where $u$ is a closed expression and $R^{\prime}, R$, resp. contains the definition of repeat, or repeat and map, resp., i.e. the corresponding $\mathbb{E}_{\mathcal{L}}$-expressions are:

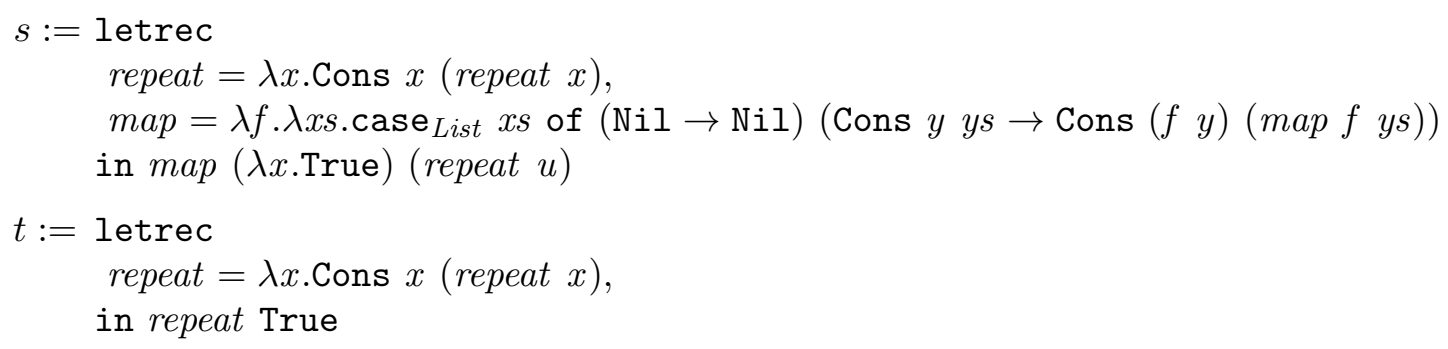

Let $\eta:=\{(t, s),(s, t)\} \cup\{($ True, True $)\}$. We show that $\eta \subseteq F_{L R, \sim}(\eta)$ which implies $s \preccurlyeq L R, \sim t$ as well as $t \preccurlyeq L R, \sim s$ and thus by Theorem 7.12 also $s \sim_{L R} t$.

Evaluating $s$ and $t$ in normal order first shows: $s \sim_{L R} v_{1}, t \sim_{L R} v_{2}$ with

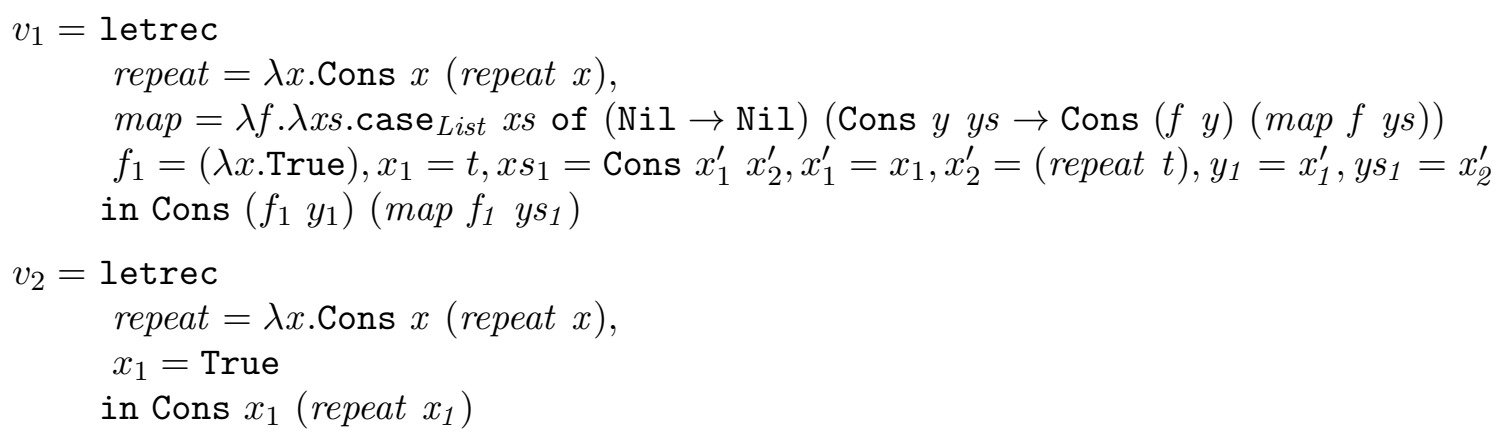

Using correctness of garbage collection, copying of bindings (gcp), shifting constructors over letrec, and the other correct reduction rules (see Theorem 3.7 and Proposition 5.34), we can simplify as follows: $v_{1} \sim_{L R}$ Cons True $s$ and $v_{2} \sim_{L R}$ Cons True $t$. Now the proof is finished, since obviously True $\eta$ True and $s \eta t, t \eta s$.

\section{Conclusion}

In this paper we have shown that co-inductive applicative bisimilarity, in the style of Howe, and also the inductive variant, is equivalent to contextual equivalence in a deterministic callby-need calculus with letrec, case, data constructors, and seq which models the (untyped) core language of Haskell. This also shows soundness of untyped applicative bisimilarity 
for the polymorphically typed variant of $L_{L R}$. As a further work one may try to establish a coincidence of the typed applicative bisimilarity and contextual equivalence for a polymorphically typed core language of Haskell.

\section{ACKNOWLEDGEMENTS}

The authors thank the anonymous reviewers for their valuable comments.

\section{REFERENCES}

[AB97] Zena M. Ariola and Stefan Blom. Cyclic lambda calculi. In Martín Abadi and Takayasu Ito, editors, TACS 1997, volume 1281 of Lecture Notes in Comput. Sci., pages 77-106. Springer, 1997.

[AB02] Z. M. Ariola and S. Blom. Skew confluence and the lambda calculus with letrec. Ann. Pure Appl. Logic, 117:95-168, 2002.

[Abr90] S. Abramsky. The lazy lambda calculus. In D. A. Turner, editor, Research Topics in Functional Programming, pages 65-116. Addison-Wesley, 1990.

[AF97] Z. M. Ariola and M Felleisen. The call-by-need lambda calculus. J. Funct. Programming, $7(3): 265-301,1997$.

$\left[\mathrm{AFM}^{+} 95\right]$ Z. M. Ariola, M. Felleisen, J. Maraist, M. Odersky, and P. Wadler. A call-by-need lambda calculus. In POPL 1995, pages 233-246. ACM, 1995.

[AK94] Z. M. Ariola and J. W. Klop. Cyclic Lambda Graph Rewriting. In LICS 1994, pages 416-425. IEEE, 1994.

[AO93] S. Abramsky and C.-H. L. Ong. Full abstraction in the lazy lambda calculus. Inf. Comput., 105(2):159-267, 1993.

[Bar84] H. P. Barendregt. The Lambda Calculus. Its Syntax and Semantics. North-Holland, Amsterdam, New York, 1984.

[DBG97] Louise A. Dennis, Alan Bundy, and Ian Green. Using a generalisation critic to find bisimulations for coinductive proofs. In CADE 1997, volume 1249 of Lecture Notes in Comput. Sci., pages 276-290, London, UK, UK, 1997. Springer-Verlag.

[Fel91] M. Felleisen. On the expressive power of programming languages. Sci. Comput. Programming, $17(1-3): 35-75,1991$.

[Gol05] M. Goldberg. A variadic extension of Curry's fixed-point combinator. Higher-Order and Symbolic Computation, 18(3-4):371-388, 2005.

[Gor99] A. D. Gordon. Bisimilarity as a theory of functional programming. Theoret. Comput. Sci., 228(12):5-47, 1999.

[How89] D. Howe. Equality in lazy computation systems. In LICS 1989, pages 198-203. IEEE, 1989.

[How96] D. Howe. Proving congruence of bisimulation in functional programming languages. Inform. and Comput., 124(2):103-112, 1996.

[Jef94] A. Jeffrey. A fully abstract semantics for concurrent graph reduction. In LICS 1994, pages 82-91. IEEE, 1994.

[JV06] P. Johann and J. Voigtländer. The impact of seq on free theorems-based program transformations. Fund. Inform., 69(1-2):63-102, 2006.

[KKSdV97] R. Kennaway, J. W. Klop, M. Ronan Sleep, and F.-J. de Vries. Infinitary lambda calculus. Theoret. Comput. Sci., 175(1):93-125, 1997.

[KW06] V. Koutavas and M. Wand. Small bisimulations for reasoning about higher-order imperative programs. In POPL 2006, pages 141-152. ACM, 2006.

[Lau93] J. Launchbury. A natural semantics for lazy evaluation. In POPL 1993, pages 144-154. ACM, 1993.

[Mil80] R. Milner. A Calculus of Communicating Systems, volume 92 of Lecture Notes in Comput. Sci. Springer, 1980.

[Mil99] R. Milner. Communicating and Mobile Systems: the $\pi$-calculus. Cambridge university press, 1999.

[Mor68] J.H. Morris. Lambda-Calculus Models of Programming Languages. PhD thesis, MIT, 1968. 
[MOW98] J. Maraist, M. Odersky, and P. Wadler. The call-by-need lambda calculus. J. Funct. Programming, 8:275-317, 1998.

[MS99] A. K. D. Moran and D. Sands. Improvement in a lazy context: An operational theory for call-by-need. In POPL 1999, pages 43-56. ACM, 1999.

[MSS10] M. Mann and M. Schmidt-Schauß. Similarity implies equivalence in a class of non-deterministic call-by-need lambda calculi. Inform. and Comput., 208(3):276 - 291, 2010.

[NH09] K. Nakata and M. Hasegawa. Small-step and big-step semantics for call-by-need. J. Funct. Program., 19:699-722, 2009.

[Pey03] S. Peyton Jones. Haskell 98 language and libraries: the Revised Report. Cambridge University Press, 2003. www.haskell.org.

[Pit97] A. M. Pitts. Operationally-based theories of program equivalence. In Semantics and Logics of Computation. Cambridge University Press, 1997.

[Pit11] A. M. Pitts. Howe's method for higher-order languages. In D. Sangiorgi and J. Rutten, editors, Advanced Topics in Bisimulation and Coinduction, volume 52 of Cambridge Tracts Theoret. Comput. Sci., chapter 5, pages 197-232. Cambridge University Press, 2011.

[Plo75] G. D. Plotkin. Call-by-name, call-by-value, and the lambda-calculus. Theoret. Comput. Sci., 1:125-159, 1975.

[Ses97] P. Sestoft. Deriving a lazy abstract machine. J. Funct. Programming, 7(3):231-264, 1997.

[SKS11] D. Sangiorgi, N. Kobayashi, and E. Sumii. Environmental bisimulations for higher-order languages. ACM Trans. Program. Lang. Syst., 33(1):5, 2011.

[SS07] M. Schmidt-Schauß. Correctness of copy in calculi with letrec. In RTA 2007, volume 4533 of Lecture Notes in Comput. Sci., pages 329-343. Springer, 2007.

[SSM08] M. Schmidt-Schauß and E. Machkasova. A finite simulation method in a non-deterministic callby-need calculus with letrec, constructors and case. In RTA 2008, number 5117 in Lecture Notes in Comput. Sci., pages 321-335. Springer-Verlag, 2008.

[SSMS13] M. Schmidt-Schauß, E. Machkasova, and D. Sabel. Extending Abramsky's lazy lambda calculus: (non)-conservativity of embeddings. In RTA 2013, volume 21 of LIPIcs, pages 239-254, Dagstuhl, Germany, 2013. Schloss Dagstuhl.

[SSNSS08] M. Schmidt-Schauß, J. Niehren, J. Schwinghammer, and D. Sabel. Adequacy of compositional translations for observational semantics. In 5th IFIP TCS 2008, volume 273 of IFIP, pages 521-535. Springer, 2008.

[SSNSS09] M. Schmidt-Schauß, J. Niehren, J. Schwinghammer, and D. Sabel. Adequacy of compositional translations for observational semantics. Frank report 33, Inst. f. Informatik, Goethe-University, Frankfurt, 2009. http://www.ki.informatik.uni-frankfurt.de/papers/frank/

[SSS11] D. Sabel and M. Schmidt-Schauß. A contextual semantics for Concurrent Haskell with futures. In PPDP 2011, pages 101-112, New York, NY, USA, 2011. ACM.

[SSS12] David Sabel and Manfred Schmidt-Schauß. Conservative concurrency in Haskell. In Nachum Dershowitz, editor, LICS 2012, pages 561-570. IEEE, 2012.

[SSSM10] M. Schmidt-Schauß, D. Sabel, and E. Machkasova. Simulation in the call-by-need lambdacalculus with letrec. In RTA 2010, volume 6 of LIPIcs, pages 295-310. Schloss Dagstuhl, 2010.

[SSSM11] M. Schmidt-Schauß, D. Sabel, and E. Machkasova. Counterexamples to applicative simulation and extensionality in non-deterministic call-by-need lambda-calculi with letrec. Inf. Process. Lett., 111(14):711-716, 2011.

[SSSS08] M. Schmidt-Schauß, M. Schütz, and D. Sabel. Safety of Nöcker's strictness analysis. J. Funct. Programming, 18(04):503-551, 2008.

[SW01] D. Sangiorgi and D. Walker. The $\pi$-calculus: a theory of mobile processes. Cambridge university press, 2001.

[VJ07] J. Voigtländer and P. Johann. Selective strictness and parametricity in structural operational semantics, inequationally. Theor. Comput. Sci, 388(1-3):290-318, 2007.

This work is licensed under the Creative Commons Attribution-NoDerivs License. To view a copy of this license, visit http://creativecommons.org/licenses/by-nd/2.0/ or send a letter to Creative Commons, 171 Second St, Suite 300, San Francisco, CA 94105, USA, or Eisenacher Strasse 2, 10777 Berlin, Germany 